\title{
A COMBINATION THEOREM FOR METRIC BUNDLES
}

\author{
MAHAN MJ AND PRANAB SARDAR
}

\begin{abstract}
We introduce the notion of metric (graph) bundles which provide a coarse-geometric generalization of the notion of trees of metric spaces a la Bestvina-Feighn in the special case that the inclusions of the edge spaces into the vertex spaces are uniform coarsely surjective quasi-isometries. We prove the existence of quasi-isometric sections in this generality. Then we prove a combination theorem for metric (graph) bundles that establishes sufficient conditions, particularly flaring, under which the metric bundles are hyperbolic. We use this to give examples of surface bundles over hyperbolic disks, whose universal cover is Gromov-hyperbolic. We also show that in typical situations, flaring is also a necessary condition.
\end{abstract}

\section{CONTENTS}

1. Introduction

1.1. Metric Bundles

1.2. Hyperbolic metric spaces

2. QI Sections

2.1. Existence of qi sections 23

2.2. Ladders

3. Construction of Hyperbolic Ladders

3.1. Hyperbolicity of ladders: Special case

3.2. Hyperbolicity of ladders: General case 43

4. The Combination Theorem 47

4.1. Proof of Proposition $4.2 \quad 49$

5. Consequences and Applications 52

5.1. Sections, Retracts and Cannon-Thurston maps 52

5.2. Hyperbolicity of base and flaring 53

5.3. Necessity of Flaring

5.4. An Example 58

References 61

\section{INTRODUCTION}

In this paper we introduce the notions of metric bundles and metric graph bundles which provide a purely coarse-geometric generalization of the notion of trees of

Date: September 19, 2018.

Research of first author partially supported by a CEFIPRA Indo-French Research grant. The second author is partly supported by a CSIR Research Fellowship. This paper is part of PS's PhD thesis written under the supervision of MM. 
metric spaces a la Bestvina-Feighn [BF92] (see Section 1.3] in the special case that the inclusions of the edge spaces into the vertex spaces are uniform coarsely surjective quasi-isometries. We generalize the base space from a tree to an arbitrary hyperbolic metric space. In [FM02, Farb and Mosher introduced the notion of metric fibrations which was used by Hamenstadt to give a combination theorem in Ham05. Metric fibrations can be thought of as metric bundles (in our terminology) equipped with a foliation by totally geodesic sections of the base space. We first prove the following Proposition which ensures the existence of q(uasi)-i(sometric) sections in the general context of metric bundles, generalizing and giving a different proof of a result due to Mosher Mos96] in the context of exact sequences of groups (see Example 1.8).

Proposition 2.10 (Existence of qi sections): Let $\delta, N \geq 0$ and suppose $p$ : $X \rightarrow B$ is an $(f, K)$-metric graph bundle with the following properties:

(1) Each of the fibers $F_{b}, b \in \mathcal{V}(B)$ is a $\delta$-hyperbolic geodesic metric space with respect to the path metric $d_{b}$ induced from $X$.

(2) The barycenter maps $\phi_{b}: \partial^{3} F_{b} \rightarrow F_{b}$ are uniformly coarsely surjective, i.e. $F_{b}$ is contained in the $N$-neighborhood of the image of $\phi_{b}$ for all $b \in \mathcal{V}(B)$.

Then there is a $K_{0}=K_{0}(f, \delta, N)$-qi section through each point of $\mathcal{V}(X)$.

Proposition 2.10 provides a context for developing a 'coarse theory of bundles' and proving the following combination theorem, which is the main theorem of this paper.

Theorem 4.3; Suppose $p: X \rightarrow B$ is a metric bundle (resp. metric graph bundle) such that

(1) $B$ is a $\delta$-hyperbolic metric space.

(2) Each of the fibers $F_{b}, b \in B$ (resp. $\left.b \in \mathcal{V}(B)\right)$ is a $\delta^{\prime}$-hyperbolic geodesic metric space with respect to the path metric induced from $X$.

(3) The barycenter maps $\partial^{3} F_{b} \rightarrow F_{b}, b \in B$ (resp. $b \in \mathcal{V}(B)$ ) are uniformly coarsely surjective.

(4) A flaring condition is satisfied.

Then $X$ is a hyperbolic metric space.

This is a first step towards proving a combination Theorem for more general complexes of spaces (cf. Problem 90 of Kap08).

Theorem 4.3 generalizes Hamenstadt's combination theorem (Corollary 3.8 of Ham05]) in two ways:

a) It removes the hypothesis of properness of the base space $B$ - a hypothesis that is crucial in Ham05 to ensure compactness of the boundary of the base space and hence allow the arguments in Ham05 to work. This generalization is relevant for two reasons. First, underlying trees in trees of spaces are frequently non-proper. Secondly, curve complexes of surfaces are mostly non-proper metric spaces and occur as natural base spaces for metric bundles. See LMS11 by Leininger-MjSchleimer for a closely related example.

b) It removes the hypothesis on existence of totally geodesic sections in Ham05 altogether. Proposition 2.10 ensures the existence of qi sections under mild technical assumptions.

A word about the proof of Theorem 4.3 ahead of time. Proposition 2.10 ensures the existence of qi sections through points of $X$. We use the notion of flaring from 
Bestvina-Feighn BF92 and a criterion for hyperbolicity introduced by Hamenstadt in Ham07. to construct certain path families and use them to prove hyperbolicity. Another crucial ingredient is a 'ladder-construction' due to the first author Mit98b, which may be regarded as an analog of the hallways of [BF92.

Recall Far98] that for a pair $(X, \mathcal{H})$ of a metric space $\left(X, d_{X}\right)$ and a family of path-connected subsets $\mathcal{H}$ of $X$, the electric space $\mathcal{E}(X, \mathcal{H})$ is the pseudo-metric space $X \sqcup_{H \in \mathcal{H}} H \times[0,1]$ with $H \times\{0\}$ identified with $H \subset X$ and $H \times\{1\}$ equipped with the zero metric. Each $\{h\} \times[0,1]$ is isometric to the unit interval. There is a natural inclusion map $E: X \rightarrow \mathcal{E}(X, \mathcal{H})$ which is referred to as the electrocution map. The image $E(X)$ inherits a metric called the electric metric $d_{e}$.

As an application of Theorem 4.3 we obtain a rather plentiful supply of examples from the following Proposition, where the base space need not be a tree (as in all previously known examples). Let $S$ be a closed surface of genus greater than one and Teich $(S)$ be the Teichmuller space of $S$. The Teichmuller metric on Teich $(S)$ is denoted as $d_{T}$ and $d_{e}$ denotes the electric metric on Teich $(S)$ obtained by electrocuting the $\alpha$-thin parts of Teich $(S)$ for every essential simple closed curve $\alpha$ on $S$. For $j: K \rightarrow\left(\operatorname{Teich}(S), d_{T}\right)$ a map, let $U(S, K)$ denote the pullback (under $j$ ) of the universal curve over Teich $(S)$ equipped with the natural path metric. Also, the universal cover of the universal curve over $T \operatorname{eich}(S)$ is a hyperbolic plane bundle over Teich $(S)$. Let $\widetilde{U(S, K)}$ denote the pullback to $K$ of this hyperbolic plane bundle.

Proposition 5.15 Let $\left(K, d_{K}\right)$ be a hyperbolic metric space satisfying the following:

There exists $C \geq 0$ such that for any two points $u, v \in K$, there exists a bi-infinite $C$-quasigeodesic $\gamma \subset K$ with $d_{K}(u, \gamma) \leq C$ and $d_{K}(v, \gamma) \leq C$.

Let $j: K \rightarrow\left(\right.$ Teich $\left.(S), d_{T}\right)$ be a quasi-isometric embedding such that $E \circ j: K \rightarrow$ $\left(\right.$ Teich $\left.(S), d_{e}\right)$ is also a quasi-isometric embedding. Then $\widetilde{U(S, K)}$ is a hyperbolic metric space.

It is an open question (cf. KL08 FM02 to find purely pseudo Anosov surface groups $Q\left(=\pi_{1}(\Sigma)\right.$, say) in $M C G(S)$. This is equivalent to constructing surface bundles over surfaces with total space $W$, fiber $S$, and base $\Sigma$, such that $\pi_{1}(W)$ does not contain a copy of $\mathbb{Z} \oplus \mathbb{Z}$. One way of ensuring this is to find an example where the total space has (Gromov) hyperbolic fundamental group $\pi_{1}(W)$. A quasi-isometric model for the universal cover $\widetilde{W}$ is a metric graph bundle where the fibers are Cayley graphs of $\pi_{1}(S)$ and the base $K$ a Cayley graph of $\pi_{1}(\Sigma)$. Using a construction of Leininger and Schleimer [LS11] in conjunction with Proposition 5.15 we construct examples of hyperbolic metric graph bundles where fibers are Cayley graphs of $\pi_{1}(S)$ and $K$ is a hyperbolic disk. However the disks $K$ are not invariant under a surface group; so we only obtain surface bundles $W$ over $K$ with fiber $S$ such that the universal cover $\widetilde{W}$ is hyperbolic.

We also obtain the following characterization of convex cocompact subgroups of mapping class groups of surfaces $S^{h}$ with punctures. Recall that the pure mapping class group is the (finite index) subgroup of the mapping class group that keeps the punctures fixed.

Proposition 5.17; Let $K=\pi_{1}\left(S^{h}\right)$ be the fundamental group of a surface with finitely many punctures and let $K_{1}, \cdots, K_{n}$ be its peripheral subgroups. Let $Q$ be a 
convex cocompact subgroup of the pure mapping class group of $S^{h}$. Let

$$
1 \rightarrow K \rightarrow G \stackrel{p}{\rightarrow} Q \rightarrow 1
$$

and

$$
1 \rightarrow K_{i} \rightarrow N_{G}\left(K_{i}\right) \stackrel{p}{\rightarrow} Q_{i} \rightarrow 1
$$

be the induced short exact sequences of groups. Then $G$ is strongly hyperbolic relative to the collection $\left\{N_{G}\left(K_{i}\right)\right\}, i=1, \cdots, n$.

Conversely, if $G$ is (strongly) hyperbolic relative to the collection $\left\{N_{G}\left(K_{i}\right)\right\}, i=$ $1, \cdots, n$, then $Q$ is convex-cocompact.

Theorem 4.3 also provides the following combination theorem whenever we have an exact sequence with hyperbolic quotient and kernel. This gives a converse to a result of Mosher Mos96.

Theorem 5.1; Suppose that the short exact sequence of finitely generated groups

$$
1 \rightarrow K \rightarrow G \rightarrow Q \rightarrow 1
$$

satisfies a flaring condition such that $K, Q$ are word hyperbolic and $K$ is nonelementary. Then $G$ is hyperbolic.

The next Proposition links the flaring condition to hyperbolicity of the base.

Proposition 5.5: Consider the short exact sequence of finitely generated groups

$$
1 \rightarrow K \rightarrow G \rightarrow Q \rightarrow 1
$$

such that $K$ is non-elementary word hyperbolic but $Q$ is not hyperbolic. Then the short exact sequence cannot satisfy a flaring condition.

We also prove an analog of Proposition 5.5 for relatively hyperbolic groups and use it to generalize a result of Mosher Mos96 as follows.

Proposition 5.7. Suppose we have a short exact sequence of finitely generated groups

$$
1 \rightarrow\left(K, K_{1}\right) \rightarrow\left(G, N_{G}\left(K_{1}\right)\right) \stackrel{p}{\rightarrow}\left(Q, Q_{1}\right) \rightarrow 1
$$

with $K$ (strongly) hyperbolic relative to the cusp subgroup $K_{1}$ such that $G$ preserves cusps and $Q_{1}=N_{G}\left(K_{1}\right) / K_{1}$. Suppose further that $G$ is (strongly) hyperbolic relative to $N_{G}\left(K_{1}\right)$. Then $Q\left(=Q_{1}\right)$ is hyperbolic.

Finally we show the necessity of flaring.

Proposition 5.8; Let $P: X \rightarrow B$ be a metric (graph) bundle such that

1) $X$ is hyperbolic.

2) There exists $\delta_{0}$ such that each fiber $F_{z}=p^{-1}(z) \subset X$ equipped with the inherited path metric is $\delta_{0}$-hyperbolic.

Then the metric bundle satisfies a flaring condition.

In particular, any exact sequence of finitely generated groups $1 \rightarrow N \rightarrow G \rightarrow$ $Q \rightarrow 1$ with $N$ and $G$ hyperbolic, satisfies a flaring condition.

Outline of the main steps:

There are four main steps in the proof of Combination Theorem 4.3. Precise definitions of terms are given in the next subsection.

1) First we construct a metric graph bundle (see Definition [1.5) out of a given metric bundle. The bundles have quasi-isometric base space and total space. Next we set out to prove that this metric graph bundle is hyperbolic under the given conditions on the metric bundle. 
2) Proposition 2.10 proves the existence of qi sections and is the coarse geometric analog of the statement that any fiber bundle with contractible base admits a section. The main ingredient of the proof is the definition of a 'discrete flow' of one fiber to another fiber. This is the content of Section 2.1. The main idea is elaborated upon in the first paragraph of Section 2.1.

3) Any two such qi sections bound a 'ladder' between them (cf. Definition 2.13 below, Mit98a, Mit98b). The next step is to prove the hyperbolicity of these ladders. In Section 3.1 we prove hyperbolicity of small-girth ladders (Proposition 3.4). In Section 3.2 we break up a big ladder into small-girth ladders and use a consequence (Proposition 1.51) of a combination theorem due to Mj-Reeves [MR08 to conclude that the whole ladder is hyperbolic.

4) In Section 4, we assemble the pieces to prove Theorem 4.3

For the reader interested in getting to the main ideas of the proof of Theorem 4.3 without getting into technical details, we have sketched Step (2) above in the first paragraph of Section 2.1, and Step (3) above in the the first paragraph of Section 3 and the paragraph following the statement of Proposition 3.4 in Section 3.1.

Acknowledgments: We would like to thank Panos Papasoglu for explaining the proof of the last statement of Theorem 5.4 to us. We would also like to thank Chris Leininger for sharing his examples in [LS11] with us. This paper owes an intellectual debt to Hamenstadt's paper Ham05, which inspired us to find a combination Theorem in the generality described here. Finally we would like to thank the referee for a meticulous reading of the manuscript and for several helpful remarks and comments. In particular, the notion of a metric graph bundle arose out of the referee's comments on an earlier draft.

\subsection{Metric Bundles.}

1.1.1. Some Basic Concepts. We recall some basic notions from large scale geometry.

Let $X, Y$ be metric spaces and let $k \geq 1, \epsilon \geq 0$.

(1) A map $\phi: X \rightarrow Y$ is said to be metrically proper if for all $N \geq 0$ there exists $M \geq 0$ such that $x, y \in X$, and $d(\phi(x), \phi(y)) \leq N$ implies $d(x, y) \leq M$.

Suppose $\left\{\left(X_{\alpha}, d_{X_{\alpha}}\right)\right\}$ and $\left\{\left(Y_{\alpha}, d_{Y_{\alpha}}\right)\right\}$ are families of metric spaces. For any function $f: \mathbb{R}^{+} \rightarrow \mathbb{R}^{+}$, a family of maps $\phi_{\alpha}: X_{\alpha} \rightarrow Y_{\alpha}$ is said to be uniformly metrically proper as measured by $f$ if for all $\alpha$ and $x, y \in X_{\alpha}, d_{Y_{\alpha}}\left(\phi_{\alpha}(x), \phi_{\alpha}(y)\right) \leq N$ implies $d_{X_{\alpha}}(x, y) \leq f(N)$. If such an $f$ exists we shall say that the collection of maps $\phi_{\alpha}$ is uniformly metrically proper or, more simply, uniformly proper.

(2) Suppose $A$ is a set. A map $\phi: A \rightarrow Y$ is said to be $\epsilon$-coarsely surjective if $Y$ is contained in the $\epsilon$-neighborhood $\phi(A)$.

Suppose $\left\{A_{\alpha}\right\}$ and $\left\{Y_{\alpha}\right\}$ are respectively a family of sets and a family of metric spaces. A family of maps $\phi_{\alpha}: A_{\alpha} \rightarrow Y_{\alpha}$ is said to be uniformly coarsely surjective if there is a constant $D \geq 0$, such that for all $\alpha, Y_{\alpha}$ is contained in the $D$-neighborhood of $\phi_{\alpha}\left(A_{\alpha}\right)$.

(3) A map $\phi: X \rightarrow Y$ is said to be $\epsilon$-coarsely Lipschitz if $\forall x_{1}, x_{2} \in X$ we have $d\left(\phi\left(x_{1}\right), \phi\left(x_{2}\right)\right) \leq \epsilon \cdot d\left(x_{1}, x_{2}\right)+\epsilon$. A map $\phi$ is coarsely Lipschitz if it is $\epsilon$ - coarsely Lipschitz for some $\epsilon \geq 1$. 
(4) (i) Recall Gro85 Gd90 that a map $\phi: X \rightarrow Y$ is said to be a $(k, \epsilon)$ quasi-isometric embedding if $\forall x_{1}, x_{2} \in X$ one has

$$
d\left(x_{1}, x_{2}\right) / k-\epsilon \leq d\left(\phi\left(x_{1}\right), \phi\left(x_{2}\right)\right) \leq k . d\left(x_{1}, x_{2}\right)+\epsilon .
$$

A map $\phi: X \rightarrow Y$ will simply be referred to as a quasi-isometric embedding if it is a $(k, \epsilon)$-quasi-isometric embedding for some $k \geq 1$ and $\epsilon \geq 0$. A $(k, k)$-quasi-isometric embedding will be referred to as a $k$-quasi-isometric embedding.

(ii) A map $\phi: X \rightarrow Y$ is said to be a $(k, \epsilon)$-quasi-isometry (resp. $k$ quasi-isometry) if it is a $(k, \epsilon)$-quasi-isometric embedding (resp. $k$-quasiisometric embedding) and if $\phi$ is $D$-coarsely surjective for some $D \geq 0$.

(iii) A $(k, \epsilon)$-quasi-geodesic (resp. a $k$-quasi-geodesic) in a metric space $X$ is a $(k, \epsilon)$-quasi-isometric embedding (resp. a $k$-quasi-isometric embedding) $\gamma: I \rightarrow X$, where $I \subseteq \mathbb{R}$ is an interval.

(5) A map $\psi: Y \rightarrow X$ is said to be an $\epsilon$-coarse inverse of a map $\phi: X \rightarrow Y$ if for all $x \in X$ and $y \in Y$ one has $d_{X}(\psi \circ \phi(x), x) \leq \epsilon$ and $d_{X}(\phi \circ \psi(y), y) \leq \epsilon$.

The following lemma is straightforward. We include a proof for the sake of completeness.

Lemma 1.1. For every $K_{1}, K_{2} \geq 1$ and $D \geq 0$ there are $K_{[1.1]}=K_{[1.1}\left(K_{1}, K_{2}, D\right)$, and $K_{1.1}^{\prime}=K_{[1.1}^{\prime}\left(K_{1}, D\right)$ such that the following hold.

(1) A $K_{1}$-coarsely Lipschitz map with a $K_{2}$-coarsely Lipschitz, D-coarse inverse is a $K_{1.1}$ quasi-isometry.

(2) Any D-coarsely surjective, $K_{1}$-quasi-isometry has a $K_{1.1}^{\prime}$ quasi-isometric coarse inverse.

Proof. 1. Let $f: X \rightarrow Y$ be a $K_{1}$-coarsely Lipschitz map with a $K_{2}$-coarsely Lipschitz, $D$-coarse inverse $g: Y \rightarrow X$. Let $x, y, x^{\prime}, y^{\prime} \in X$ be such that $g(f(x))=$ $x^{\prime}, g(f(y))=y^{\prime}$. Since $g$ is a $D$-coarse inverse of $f$, we have $d\left(x, x^{\prime}\right) \leq D, d\left(y, y^{\prime}\right) \leq$ $D$. Now, $-d\left(x, x^{\prime}\right)-d\left(y, y^{\prime}\right)+d(x, y) \leq d\left(x^{\prime}, y^{\prime}\right) \leq K_{2} d(f(x), f(y))+K_{2}$. Hence, $-2 D+d(x, y) \leq K_{2} d(f(x), f(y))+K_{2}$. Choosing $K_{1.1}=\max \left\{K_{1}, 2 D+K_{2}\right\}$ completes the proof.

2. Suppose $f: X \rightarrow Y$ is a $D$-coarsely surjective, $K_{1}$-quasi-isometry. We define a map $g: Y \rightarrow X$ as follows: For all $v \in Y$, choose $x \in X$ such that $d(v, f(x)) \leq D$. Define $g(v)=x$. Let $v_{1}, v_{2} \in Y$ and let $g\left(v_{i}\right)=x_{i}, i=1,2$. Then $d\left(v_{i}, f\left(x_{i}\right)\right) \leq D$, $i=1,2$. It follows that $\left|d\left(f\left(x_{1}\right), f\left(x_{2}\right)\right)-d\left(v_{1}, v_{2}\right)\right| \leq 2 D$. Again, since $f$ is a $K_{1}$ quasi-isometry, we have $-K_{1}+\frac{1}{K_{1}} d\left(x_{1}, x_{2}\right) \leq d\left(f\left(x_{1}\right), f\left(x_{2}\right)\right) \leq K_{1}+K_{1} d\left(x_{1}, x_{2}\right)$. We deduce from the previous two inequalities that $-\left(K_{1}+2 D\right)+\frac{1}{K_{1}} d\left(x_{1}, x_{2}\right) \leq$ $d\left(v_{1}, v_{2}\right) \leq\left(K_{1}+2 D\right)+K_{1} d\left(x_{1}, x_{2}\right)$. Hence finally, we have

$$
-\frac{\left(K_{1}+2 D\right)}{K_{1}}+\frac{1}{K_{1}} d\left(v_{1}, v_{2}\right) \leq d\left(x_{1}, x_{2}\right) \leq K_{1} d\left(v_{1}, v_{2}\right)+\left(K_{1}+2 D\right) K_{1} .
$$

Thus $g$ is a $K_{1.1}^{\prime}$ quasi-isometric embedding where $K_{1.1}^{\prime}=K_{1}\left(K_{1}+2 D\right)$.

It follows from the definition of $g$ that for all $v \in Y$, one has $d(f(g(v)), v) \leq D$. Let $x \in X$ and $g(f(x))=x_{1}$. Hence $d\left(f(x), f\left(x_{1}\right)\right) \leq D$. Since $f$ is a $K_{1}$-quasiisometric embedding, it follows that $d(g(f(x)), x)=d\left(x, x_{1}\right) \leq K_{1}\left(K_{1}+D\right)$. Thus $g$ is $K_{1}\left(K_{1}+D\right)$-coarsely surjective whence a $K_{1.1}^{\prime}$ quasi-isometry. Also $g$ is a $K_{1}\left(K_{1}+D\right)$-coarse inverse of $f$. 
1.1.2. Metric Bundles and Metric Graph Bundles. In this subsection we define the primary objects of study and obtain some basic properties.

Definition 1.2. Suppose $(X, d)$ and $\left(B, d_{B}\right)$ are geodesic metric spaces; let $c \geq 1$ and let $f: \mathbb{R}^{+} \rightarrow \mathbb{R}^{+}$be a function. We say that $X$ is an $(f, c)$ - metric bundle over $B$ if there is a surjective 1-Lipschitz map $p: X \rightarrow B$ such that the following conditions hold:

1) For each point $z \in B, F_{z}:=p^{-1}(z)$ is a geodesic metric space with respect to the path metric $d_{z}$ induced from $X$. The inclusion maps $i:\left(F_{z}, d_{z}\right) \rightarrow X$ are uniformly metrically proper as measured by $f$.

2) Suppose $z_{1}, z_{2} \in B, d_{B}\left(z_{1}, z_{2}\right) \leq 1$ and let $\gamma$ be a geodesic in $B$ joining them.

$2(i)$ Then for any point $x \in F_{z}, z \in \gamma$, there is a path in $p^{-1}(\gamma)$ of length at most $c$ joining $x$ to both $F_{z_{1}}$ and $F_{z_{2}}$.

Remark 1.3. Since the metric on each fiber $F_{z}, z \in B$ is the path metric induced from $X$ we always have $f(t) \geq t$ for all $t \in \mathbb{R}^{+}$.

Convention: We shall use subscripts for constants to indicate the Lemma/ Proposition/Theorem/Corollary where they first appear.

Proposition 1.4. Let $X$ be an $(f, c)$ - metric bundle over $B$. Then there exists $K_{1.4}=K_{1.4}(f, c) \geq 1$, such that the following holds.

Suppose $z_{1}, z_{2} \in B$ with $d_{B}\left(z_{1}, z_{2}\right) \leq 1$ and let $\gamma$ be a geodesic in $B$ joining them. Let $\phi: F_{z_{1}} \rightarrow F_{z_{2}}$, be any map such that $\forall x_{1} \in F_{z_{1}}$ there is a path of length at most c in $p^{-1}(\gamma)$ joining $x_{1}$ to $\phi\left(x_{1}\right)$. Then $\phi$ is a $K_{1.4}$ quasi-isometry.

Proof. Let $u, v \in F_{z_{1}}$ such that $d_{z_{1}}(u, v) \leq 1$. Then $d(\phi(u), \phi(v)) \leq 2 c+1$ by the triangle inequality and hence $d_{z_{2}}(\phi(u), \phi(v)) \leq f(2 c+1)$ by condition $2(\mathrm{i})$ of the definition of metric bundles. It follows that the map $\phi$ is an $f(2 c+1)$-coarsely Lipschitz map. A similar map $\bar{\phi}: F_{z_{2}} \rightarrow F_{z_{1}}$ may be defined, appealing again to condition $2(i)$ of the definition of metric bundles, interchanging the roles of $z_{1}, z_{2}$ such that $\bar{\phi}$ is also an $f(2 c+1)$-coarsely Lipschitz map.

Also, $\bar{\phi}$ is a coarse inverse of $\phi$ : $d(\bar{\phi} \circ \phi(u), u) \leq d(\bar{\phi} \circ \phi(u), \phi(u))+d(\phi(u), u) \leq 2 c$ and hence $d_{z_{1}}(\bar{\phi} \circ \phi(u), u) \leq f(2 c)$; similarly $d_{z_{2}}(\phi \circ \bar{\phi}(v), v) \leq f(2 c)$ for all $u \in F_{z_{1}}, v \in F_{z_{2}}$.

Hence by Lemma $1.1(1), \phi$ is a $K_{[1.4}$ quasi-isometry where $K_{1.4}=K_{1.1}(f(2 c+$ $1), f(2 c+1), f(2 c))$. Note further that $\phi$ is $f(2 c)$-coarsely surjective.

We will find it convenient to refer to an $(f, c)$ - metric bundle as an $(f, c, K)$ metric bundle (with $K=K[1.4(f, c)$ ), or simply a metric bundle when the parameters are not important, and refer to the conclusion of the above proposition as Condition 2(ii) of Definition 1.2 of metric bundles.

For the rest of the paper by a graph we will always mean a connected metric graph all of whose edges are of length 1 . For a graph $X, \mathcal{V}(X)$ will denote its vertex set. By a path in a graph we will always mean an edge path starting and ending at two vertices.

Definition 1.5. Suppose $X$ and $B$ are graphs. Let $f: \mathbb{N} \rightarrow \mathbb{N}$ be a function.

We say that $X$ is an $f$ - metric graph bundle over $B$ if there exists a surjective simplicial map $p: X \rightarrow B$ such that:

1. For each $b \in \mathcal{V}(B), F_{b}:=p^{-1}(b)$ is a connected subgraph of $X$ and the inclusion maps $i: \mathcal{V}\left(F_{b}\right) \rightarrow X$ are uniformly metrically proper (as measured by $f$ ) for the path 
metric $d_{b}$ induced on $F_{b}$, i.e. for all $b \in \mathcal{V}(B)$ and $x, y \in \mathcal{V}\left(F_{b}\right), d(i(x), i(y)) \leq N$ implies that $d_{b}(x, y) \leq f(N)$.

2. Suppose $b_{1}, b_{2} \in \mathcal{V}(B)$ are adjacent vertices.

$2(i)$. Then each vertex $x_{1}$ of $F_{b_{1}}$ is connected by an edge with a vertex in $F_{b_{2}}$.

Remark 1.6. Since the map $p$ is simplicial it follows that it is 1-Lipschitz.

Now, we have the following analog of Proposition 1.4

Proposition 1.7. Suppose $X$ is an $f$-metric graph bundle over $B$. Then there exists $K_{1.7}=K_{1.7}(f) \geq 1$ such that the following holds.

Suppose $b_{1}, b_{2} \in \mathcal{V}(B)$ are adjacent vertices. Let $\phi: F_{b_{1}} \rightarrow F_{b_{2}}$ be any map such that each $x_{1} \in \mathcal{V}\left(F_{b_{1}}\right)$ is connected to $\phi\left(x_{1}\right) \in \mathcal{V}\left(F_{b_{2}}\right)$ by an edge, and any interior point on an edge of $F_{b_{1}}$ is sent to the image of one of the vertices on which the edge is incident. Then any such $\phi$ is a K 1.7 -quasi-isometry.

Proof. First note that $d_{b_{1}}(u, v) \leq 1$ implies that $d_{X}(\phi(u), \phi(v)) \leq 4$ by the triangle inequality. Hence $d_{b_{2}}(\phi(u), \phi(v)) \leq f(4)$ since $X$ is an $f$ - metric graph bundle. Thus $\phi$ is an $f(4)$-coarsely Lipschitz map.

Let $\bar{\phi}: F_{b_{2}} \rightarrow F_{b_{1}}$ be an analogous map defined by interchanging the roles of $b_{1}$ and $b_{2}$. As in the proof of Proposition 1.4 we see that $\bar{\phi}$ is an $f(3)$-coarsely surjective, $f(4)$-coarsely Lipschitz, $f(3)$-coarse inverse of $\phi$. Thus $\phi$ is a $K_{1.7}=$ $K[1.1(f(4), f(4), f(3))$-quasi-isometry (by Lemma $1.1(1)$ ).

Note also that $\phi$ is an $f(3)$-coarsely surjective map.

We will find it convenient to refer to an $f$-metric graph bundle as an $(f, K)$ metric graph bundle (with $K=K[1.7(f)$ ), or simply as a metric graph bundle when $f, K$ are understood, and refer to the conclusion of the above proposition as Condition 2(ii) of Definition 1.5 .

For both metric bundles and metric graph bundles the spaces $\left(F_{z}, d_{z}\right), z \in B$ or $z \in \mathcal{V}(B)$, will be referred to as horizontal spaces or fibers and the distance between two points in $F_{z}$ will be referred to as their horizontal distance. (Here we have the mental picture that the bundle projection maps go from left to right, and identify fibers to points.) A geodesic in $F_{z}$ will be called a horizontal geodesic. The spaces $X$ and $B$ will be referred to as the total space and the base space respectively. By a statement of the form ' $X$ is a metric bundle (resp. metric graph bundle)' we will mean that it is the total space of a metric bundle (resp. metric graph bundle).

A principal motivational example is the following.

Example 1.8. Suppose we have an exact sequence of finitely generated groups

$$
1 \rightarrow N \stackrel{i}{\rightarrow} G \stackrel{\pi}{\rightarrow} Q \rightarrow 1 .
$$

This naturally gives rise to a metric graph bundle as follows. Choose a finite symmetric generating set $S$ of $G$ such that $S$ contains a symmetric generating set $S_{1}$ of $N$. Let $X=\Gamma(G, S)$ be the Cayley graph of $G$ with respect to the generating set $S$. Let $T=(\pi(S) \backslash\{1\})$ and $B:=\Gamma(Q, T)$ be the Cayley graph of the group $Q$ with respect to the generating set $T$.

Then the map $\pi$ naturally induces a simplicial map $\pi: X \rightarrow B$ between Cayley graphs. In fact, $\pi$ maps an edge connecting two vertices of $X$ to a vertex of $B$ iff the vertices are both contained in the same coset of $N$ in $G$ and $\pi$ maps any edge connecting two distinct cosets of $N$ isometrically onto an edge of $B$. Define 
$f: \mathbb{N} \rightarrow \mathbb{N}$ as follows: $f(n)=$ number of vertices of $\Gamma(N, N \cap S)$ contained in the $n$-ball of $X$ about the identity element $1_{G}$ of $G \subset X$. Note that $\Gamma(N, N \cap S)$ is the inverse image of the identity element of $Q \subset B$ under $\pi$. Since the inverse images of the vertices of $B$ under $\pi$ are translates of the Cayley graph $\Gamma(N, N \cap S)$ under left multiplication by elements of $G$, condition 1 of Definition [1.5] is satisfied.

Condition 2(i) may be verified as follows: Let $\pi\left(g_{i} N\right)=v_{i} \in Q, i=1,2$. Suppose $v_{1}, v_{2}$ are adjacent vertices of $B$. Then there exist $n_{1}, n_{2} \in N$ such that $g_{1} n_{1}$ and $g_{2} n_{2}$ are connected by an edge in $X$. Thus $s=\left(g_{1} n_{1}\right)^{-1} g_{2} n_{2} \in S$. Hence for any element $n \in N, g_{1} n$ is connected to $g_{1} n . s=\left(g_{1} \cdot n \cdot n_{1}^{-1} g_{1}^{-1}\right) g_{2} n_{2}$ and $g_{1} n . s$ is contained in $g_{2} N$ since $N$ is a normal subgroup of $G$. Thus we have a metric graph bundle structure on $X$ over $B$.

Another simple example to keep in mind is the following.

Example 1.9. Let $X=\mathbb{H}^{2}$ and $B=\mathbb{R}$. Identify $B$ with a bi-infinite geodesic $\gamma \subset X$ with endpoints $a, b$ on the ideal boundary. Through $x \in \gamma$, let $F_{x}$ be the unique horocycle based at $a$. Define $p: X \rightarrow B$ by $p\left(F_{x}\right)=x$. This gives rise to a metric bundle structure on $X$ over $B$. Note that each $F_{x}$, equipped with the induced path-metric, is abstractly isometric to $\mathbb{R}$.

A more interesting set of examples is furnished by Proposition 5.15 towards the end of the paper.

Definition 1.10. Let $p: X \rightarrow B$ be a metric bundle (resp. metric graph bundle) and $k \geq 1$. Then $X_{1} \subseteq X$ is said to be a $k-\mathbf{q}$ (uasi)-i(sometric) section of $B$, if there is a $k$-quasi-isometric embedding $s: B \rightarrow X$ (resp. $s: \mathcal{V}(B) \rightarrow \mathcal{V}(X))$ such that $p \circ s=I d$ (resp. $p \circ s=I d$ on $\mathcal{V}(B)$ ) and $X_{1}=\operatorname{Im}(s)$.

If $X_{1}$ is a $k$-qi section and $x \in X_{1}$, then we say that $X_{1}$ is a $k$-qi section through $x$. Also, $X_{1} \subset X$ is said to be a qi section if it is a $k$-qi section for some $k \geq 1$.

Definition 1.11. Let $\gamma: I \rightarrow B$ be a geodesic, where $I \subseteq \mathbb{R}$ is an interval. By a $k$-qi lift of $\gamma$ in $X$, we mean a $k$-quasi isometric embedding $\tilde{\gamma}: I \rightarrow X$ such that $p \circ \tilde{\gamma}=\gamma$ (with the pro viso that for a metric graph bundle, $I$ is of the form $[0, n]$ for some $n \in \mathbb{N}$, and the equality $p \circ \tilde{\gamma}=\gamma$ holds only at the integer points).

Suppose $X_{1} \subseteq X$ is a $k$-qi-section and $\gamma: I \rightarrow B$ is a geodesic. By the $k$-qi lift of $\gamma$ in $X_{1}$ we mean a $k$-qi lift of $\gamma$ whose image is contained in $X_{1}$.

Definition 1.12. Suppose $p: X \rightarrow B$ is a metric bundle or a metric graph bundle. We say that it satisfies a flaring condition if for all $k \geq 1$, there exist $\lambda_{k}>1$ and $n_{k}, M_{k} \in \mathbb{N}$ such that the following holds:

Let $\gamma:\left[-n_{k}, n_{k}\right] \rightarrow B$ be a geodesic and let $\tilde{\gamma}_{1}$ and $\tilde{\gamma_{2}}$ be two $k$-qi lifts of $\gamma$ in $X$. If $d_{\gamma(0)}\left(\tilde{\gamma}_{1}(0), \tilde{\gamma_{2}}(0)\right) \geq M_{k}$, then we have

$$
\lambda_{k} \cdot d_{\gamma(0)}\left(\tilde{\gamma_{1}}(0), \tilde{\gamma_{2}}(0)\right) \leq \max \left\{d_{\gamma\left(n_{k}\right)}\left(\tilde{\gamma_{1}}\left(n_{k}\right), \tilde{\gamma_{2}}\left(n_{k}\right)\right), d_{\gamma\left(-n_{k}\right)}\left(\tilde{\gamma}_{1}\left(-n_{k}\right), \tilde{\gamma}_{2}\left(-n_{k}\right)\right)\right\} .
$$

Lemma 1.13. Given a function $f: \mathbb{N} \rightarrow \mathbb{N}$ there is a function $g: \mathbb{R}^{+} \rightarrow \mathbb{R}^{+}$such that the following holds:

Suppose $X$ is an $(f, K)$-metric graph bundle over $B$ and $b_{1}, b_{2} \in \mathcal{V}(B)$ with $d\left(b_{1}, b_{2}\right)=1$. Let $C \geq 0$ and let $\phi: F_{b_{1}} \rightarrow F_{b_{2}}$ be any map such that $\forall x_{1} \in F_{b_{1}}$, $\phi\left(x_{1}\right) \in \mathcal{V}\left(F_{b_{2}}\right)$ and $d\left(x_{1}, \phi\left(x_{1}\right)\right) \leq C$. Then $\phi$ is a $f(2[C]+1)$-Lipschitz map when restricted to $\mathcal{V}\left(F_{b_{1}}\right)$; also $\phi$ is a $g(C)$-quasi-isometry (here $[C]$ is the integer part of C). 
Proof. Suppose $z_{1}, z_{2} \in \mathcal{V}\left(F_{b_{1}}\right)$ are adjacent vertices. Then $d\left(\phi\left(z_{1}\right), \phi\left(z_{2}\right)\right) \leq$ $d\left(z_{1}, \phi\left(z_{1}\right)\right)+d\left(z_{2}, \phi\left(z_{2}\right)\right)+d\left(z_{1}, z_{2}\right) \leq 2[C]+1$. since $d\left(z_{j}, \phi\left(z_{j}\right)\right), j=1,2$ are integers by the definition of $\phi$. Thus $d_{b_{2}}\left(\phi\left(z_{1}\right), \phi\left(z_{2}\right)\right) \leq f(2[C]+1)$. The first conclusion follows.

Let $\phi_{0}: F_{b_{1}} \rightarrow F_{b_{2}}$ be a map such that each $x \in \mathcal{V}\left(F_{b_{1}}\right)$ is connected to $\phi_{0}(x) \in$ $\mathcal{V}\left(F_{b_{2}}\right)$ by an edge, and any interior point on an edge of $F_{b_{1}}$ is sent to the image of one of the vertices on which the edge is incident. We note that $d\left(x, \phi_{0}(x)\right) \leq 2$ for all $x \in F_{b_{1}}$. Also, condition $2(i i)$ says that $\phi_{0}$ is a $K$-quasi-isometry. Now, $d\left(\phi_{0}(x), \phi(x)\right) \leq d\left(\phi_{0}(x), x\right)+d(x, \phi(x))$ and so $d\left(\phi_{0}(x), \phi(x)\right) \leq[C]+2$, for all $x \in F_{b_{1}}$. Hence $d_{b_{2}}\left(\phi_{0}(x), \phi(x)\right) \leq f([C]+2)$, for all $x \in F_{b_{1}}$. We know that any map which is at a distance at most $R$ from a $K$-quasi-isometry is a $(K+2 R)$-quasiisometry. Choosing $g(C)$ to be $K+2 f([C]+2)$ concludes the proof.

\section{Bounded flaring condition for metric graph bundles}

Corollary 1.14. For all $k \in \mathbb{R}, k \geq 1$ there is a function $\mu_{k}: \mathbb{N} \rightarrow[1, \infty)$ such that the following holds:

Suppose $X$ is an $(f, K)$-metric graph bundle with base space $B$. Let $\gamma \subset B$ be a geodesic joining $b_{1}, b_{2} \in \mathcal{V}(B)$, and let $\tilde{\gamma}_{1}, \tilde{\gamma}_{2}$ be two $k$-qi lifts of $\gamma$ in $X$ which join the pairs of points $\left(x_{1}, x_{2}\right)$ and $\left(y_{1}, y_{2}\right)$ respectively, so that $p\left(x_{i}\right)=p\left(y_{i}\right)=b_{i}$, $i=1,2$. For all $N \in \mathbb{N}$, if $d_{B}\left(b_{1}, b_{2}\right) \leq N$ then

$$
d_{b_{1}}\left(x_{1}, y_{1}\right) \leq \mu_{k}(N) \max \left\{d_{b_{2}}\left(x_{2}, y_{2}\right), 1\right\} .
$$

Proof. Let $b_{1}=v_{0}, v_{1}, \cdots, v_{n}=b_{1}$ be the sequence of consecutive vertices on the geodesic $\gamma$. We must have $n \leq N$. Define for all $i=0,1, \cdots n-1, \phi_{i}: F_{v_{i}} \rightarrow F_{v_{i+1}}$ by appealing to condition $2(i)$ of the definition of metric graph bundles such that $\phi_{i}\left(\tilde{\gamma}_{j}(i)\right)=\tilde{\gamma}_{j}(i+1), j=1,2$. By the first conclusion of Lemma 1.13 each $\phi_{i}$ is $f(2[2 k]+1)$-Lipschitz when restricted to $\mathcal{V}\left(F_{v_{i}}\right)$.

Choosing $\mu_{k}(N)=f(2[2 k]+1)^{N}$ concludes the proof.

Lemma 1.13 has an obvious analog for any $(f, c)$-metric bundle. The same applies to Corollary 1.14 as well. Since the proofs are very similar we omit them.

Lemma 1.15. Given a function $f: \mathbb{R}^{+} \rightarrow \mathbb{R}^{+}$and $c \geq 0$ there is a function $g: \mathbb{R}^{+} \rightarrow \mathbb{R}^{+}$such that the following holds:

Suppose $X$ is an $(f, c, K)$-metric bundle over $B$ and $b_{1}, b_{2} \in B$ with $d_{B}\left(b_{1}, b_{2}\right) \leq$ 1. Let $C \geq 0$ and let $\phi: F_{b_{1}} \rightarrow F_{b_{2}}$ be any map such that $\forall x_{1} \in F_{b_{1}}, d\left(x_{1}, \phi\left(x_{1}\right)\right) \leq$ $C$. Then $\phi$ is a $g(C)$-quasi-isometry.

\section{Bounded flaring condition for metric bundles}

Corollary 1.16. For all $k \in \mathbb{R}^{+}, k \geq 1$ there is a function $\mu_{k}: \mathbb{R}^{+} \rightarrow[1, \infty)$ such that the following holds:

Suppose $X$ is an $(f, c, K)$-metric bundle with base space $B$. Let $\gamma \subset B$ be a geodesic joining $b_{1}, b_{2} \in B$, and let $\tilde{\gamma_{1}}, \tilde{\gamma}_{2}$ be two k-qi lifts of $\gamma$ in $X$ which join the pairs of points $\left(x_{1}, x_{2}\right)$ and $\left(y_{1}, y_{2}\right)$ respectively, so that $p\left(x_{i}\right)=p\left(y_{i}\right)=b_{i}, i=1,2$. For all $N \in \mathbb{R}^{+}$, if $d_{B}\left(b_{1}, b_{2}\right) \leq N$ then

$$
d_{b_{1}}\left(x_{1}, y_{1}\right) \leq \mu_{k}(N) \max \left\{d_{b_{2}}\left(x_{2}, y_{2}\right), 1\right\} .
$$

In the rest of the paper, we will summarize the conclusion of Corollaries 1.14 and 1.16 by saying that a metric bundle or a metric graph bundle satisfies a bounded flaring condition. 
We end this subsection by showing that a metric bundle naturally gives rise to a metric graph bundle, such that the respective base and total spaces are quasiisometric. But first, we recall the general fact that geodesic metric spaces are quasi-isometric to connected graphs (see Gro93 p.7 or [BH99] p. 152).

Lemma 1.17. 1. Let $Y$ be a geodesic metric space and let $V \subset Y$ be a subset such that for some $D>0$ and all $y \in Y$ there exists $z \in V$ such that $d(y, z) \leq D$. Let $E \geq 2 D+1$. Let $Z$ be a graph such that

a) the vertex set $\mathcal{V}(Z)=V$

b) the edge $\operatorname{set} \mathcal{E}(Z)$ is given by $\{y, z\} \in \mathcal{E}(Z)$ iff $y \neq z$ and $d(y, z) \leq E$.

Define $\psi_{Z}: Z \rightarrow Y$ as follows: $\psi_{Z}(u)=u$ for $u \in V$. For an edge e of $Z$ choose some $u \in V$ such that $e$ is incident on $u$ and map the interior of e to $u$ under $\psi_{Z}$. Then for all $u, v \in V$ we have $-1+d_{Z}(u, v) \leq d_{Y}(u, v) \leq E . d_{Z}(u, v)$. In particular, $\psi_{Z}$ is a $\max \{5,4 E\}$-quasi-isometry.

2. Suppose $Z_{1}$ is a connected subgraph of a graph $Z$ such that the vertex sets of $Z_{1}, Z$ are the same and the following holds: Let $E_{1}>1$ and suppose any edge of $Z$ which is not in $Z_{1}$ connects two vertices of $Z_{1}$ which are at a distance of at most $E_{1}$ in $Z_{1}$. Then for all $u, v \in Z_{1}$ we have $d_{Z}(u, v) \leq d_{Z_{1}}(u, v) \leq E_{1} d_{Z}(u, v)$. In particular the inclusion $Z_{1} \hookrightarrow Z$ is an $E_{1}$-quasi-isometry.

Now, suppose $p: X^{\prime} \rightarrow B^{\prime}$ is an $(f, c, K)$-metric bundle. Let $d$ denote the metric on $X^{\prime}$ and let $d_{B^{\prime}}$ be the metric on $B^{\prime}$. Let $V \subset B^{\prime}$ be a maximal subset such that $u, v \in V, u \neq v$ implies $d_{B^{\prime}}(u, v) \geq 1$. Then for all $b \in B^{\prime}$ there exists $u \in V$ such that $d_{B^{\prime}}(b, u) \leq 1$. Using the recipe of Lemma 1.17 (1) construct

a) a graph $B$ with vertex set $V$ such that $u \neq v \in V$ are connected by an edge iff $d_{B^{\prime}}(u, v) \leq 3$,

b) and a quasi-isometry $\psi_{B}: B \rightarrow B^{\prime}$.

Next, for all $u \in V$ let $X_{u}^{\prime}$ be a maximal subset of the horizontal space $F_{u}$ such that for $x, y \in X_{u}^{\prime}, d_{u}(x, y) \geq 1$.

Lemma 1.18. 1. For all $x \in X^{\prime}$ there exists $u \in V$ and a path of length at most $c+1$ connecting $x$ to a point of $X_{u}^{\prime}$.

2. If $u, v \in V$ are connected by an edge in $B$ then each point of $X_{u}^{\prime}$ is connected to a point of $X_{v}^{\prime}$ by a path in $X^{\prime}$ of length at most $3 c+1$.

Proof. Both statements follow from condition $2(i)$ of the definition of metric bundles.

Now construct a graph $X^{\prime \prime}$ with vertex set $\mathcal{V}\left(X^{\prime \prime}\right)=\cup_{u \in V} X_{u}^{\prime}$ and edge set $\mathcal{E}\left(X^{\prime \prime}\right)=\left\{\{x, y\}: x \neq y \in \mathcal{V}\left(X^{\prime \prime}\right), d(x, y) \leq 6 c+3\right\}$.

Let $X \subset X^{\prime \prime}$ be the subgraph of $X^{\prime \prime}$ such that $\mathcal{V}(X)=\mathcal{V}\left(X^{\prime \prime}\right)$ and any edge $(x, y) \in \mathcal{E}\left(X^{\prime \prime}\right)$ also belongs to $\mathcal{E}(X)$ iff

a) either $x, y \in X_{u}^{\prime}$ for some $u \in V$

b) or $x \in X_{u}^{\prime}$ and $y \in X_{v}^{\prime}$ with $d_{B}(u, v)=1$.

Let $\psi_{X}: X \rightarrow X^{\prime}$ be a map as in Lemma 1.17(1) defined by setting $\psi_{X}(x)=x$ for $x \in \cup_{u \in V} X_{u}^{\prime}$. Then $p \circ \psi_{X}=\psi_{B} \circ \pi$ on $\cup_{u \in V} X_{u}^{\prime}$. Let $\psi_{X}$ again denote an extension of this map over edges of $X$ by sending the interior of any edge to a vertex on which it is incident consistently ensuring that $p \circ \psi_{X}=\psi_{B} \circ \pi$.

For all $u \in V$ let us denote by $H_{u}$ the graph with vertex set $X_{u}^{\prime}$ and $\mathcal{E}\left(H_{u}\right):=$ $\left\{e \in \mathcal{E}(X): e\right.$ connects two elements of $\left.X_{u}^{\prime}\right\}$. 
Lemma 1.19. There is a constant $C$ such that the maps $H_{u} \rightarrow F_{u}$ obtained by restricting $\psi_{X}$ are $C$-quasi-isometries.

Proof. First of all, $H_{u}$ is a connected graph by Lemma 1.17 (1). Next, for all $u \in V$, let $\bar{H}_{u}$ be the graph with vertex set $X_{u}^{\prime}$ and edge set $\mathcal{E}\left(\bar{H}_{u}\right):=\{e \in$ $\mathcal{E}\left(H_{u}\right), e$ connects $\left.x, y \in X_{u}^{\prime}: d_{u}(x, y) \leq f(6 c+3)\right\}$. Then $H_{u}$ is a subgraph of $\bar{H}_{u}$.

Let us consider an extension of the map $H_{u} \rightarrow F_{u}$ to a map $\bar{H}_{u} \rightarrow F_{u}$ satisfying the properties of Lemma 1.17 (1). Such a map is, therefore, a quasi-isometry. By Lemma 1.17 (2) the inclusion map $H_{u} \hookrightarrow \bar{H}_{u}$ is also a quasi-isometry. Since the map $H_{u} \rightarrow F_{u}$ is the composition of quasi-isometries $H_{u} \hookrightarrow \bar{H}_{u}$ and $\bar{H}_{u} \rightarrow F_{u}$, the lemma follows.

Lemma 1.20. $\psi_{X}: X \rightarrow X^{\prime}$ is a quasi-isometry.

Proof. Let $\psi_{X^{\prime \prime}}: X^{\prime \prime} \rightarrow X^{\prime}$ be an extension of the map $\psi_{X}: X \rightarrow X^{\prime}$ with the property of Lemma 1.17 (1). By Lemma 1.17 (1) the map $\psi_{X^{\prime \prime}}: X^{\prime \prime} \rightarrow X^{\prime}$ is a $2(6 c+3)$-quasi-isometry.

Next we show that the inclusion $X \hookrightarrow X^{\prime \prime}$ is a quasi-isometry. For this suppose $x, y \in \mathcal{V}(X)$ are connected by an edge in $X^{\prime \prime}$. Suppose $x \in X_{u}^{\prime}, y \in X_{v}^{\prime}, u, v \in V$. Then $d_{B^{\prime}}(u, v) \leq d(x, y) \leq 6 c+3$. Thus $u, v$ can be joined by a path of length at most $6 c+4$, by Lemma 1.17 (1). Thus $x$ can be joined to a point $z \in X_{v}^{\prime}$ by an edge path in $X$ of length at most $6 c+4$. It follows that $d(x, z) \leq(3 c+1)(6 c+4)$. Thus $d(y, z) \leq 1+(3 c+1)(6 c+4)=D$, say. Hence $d_{v}(y, z) \leq f(D)$. Using the previous lemma we have $d_{H_{v}}(y, z) \leq C(C+f(D))$. Since $H_{v}$ is a subgraph of $X$, we have $d_{X}(y, z) \leq C(C+f(D))$. Thus $d_{X}(x, y) \leq d_{X}(x, z)+d_{X}(y, z) \leq$ $(6 c+4)+C(C+f(D))$. Lemma 1.17 (2) now shows that the inclusion $X \hookrightarrow X^{\prime \prime}$ is a quasi-isometry..

Since $\psi_{X}: X \rightarrow X^{\prime}$ is the composition of the quasi-isometries $\psi_{X^{\prime \prime}}: X^{\prime \prime} \rightarrow X^{\prime}$ and $X \hookrightarrow X^{\prime \prime}$, the lemma follows.

Define $\pi: X \rightarrow B$ by sending edges connecting any two vertices of $X_{u}^{\prime}$ (for some $u \in V)$ to $u$. Any other edge in $X$ must join vertices $x \in X_{u}^{\prime}$ and $y \in X_{v}^{\prime}$ for some $X_{u}^{\prime}, X_{v}^{\prime}$ with $d_{B}(u, v)=1$. On any such edge $[x, y], \pi$ is defined to be an isometry onto the edge $[u, v]$. Now we have the following.

Lemma 1.21. The map $\pi: X \rightarrow B$ gives a metric graph bundle.

Proof. By definition $\pi$ is a surjective, simplicial map. We check the conditions of the definition of metric graph bundles.

Condition 2(i) follows from Lemma 1.18 (2) and the definition of the graph $X$.

Let us check condition 1 now. Note that for all $u \in \mathcal{V}(B), \pi^{-1}(u)$ is the graph $H_{u}$. By Lemma 1.19, $\pi^{-1}(u)$ is a connected subgraph of $X, C$-quasi-isometric to $F_{u}$. Let $x, y \in \mathcal{V}\left(\pi^{-1}(u)\right)$. Suppose $d_{X}(x, y) \leq N, N \in \mathbb{N}$. Then $d(x, y) \leq N(6 c+3)$. Since $p: X^{\prime} \rightarrow B^{\prime}$ is an $(f, c, K)$-metric bundle it follows that $d_{u}(x, y) \leq f(N(6 c+3))$. Hence $d_{H_{u}}(x, y) \leq C . f(N(6 c+3))+C$. Defining $g(N)=[C . f(N(6 c+3))+C]$, we see that condition 1 of the definition of a metric graph bundle is satisfied.

Note: In the rest of the paper we shall assume that the maps $\psi_{X}, \psi_{B}$ are $K_{1}$ quasi-isometries. We shall refer to $\pi: X \rightarrow B$ above as an approximating metric graph bundle of the metric bundle $p: X^{\prime} \rightarrow B^{\prime}$. 
1.2. Hyperbolic metric spaces. We assume that the reader is familiar with the basic definitions and facts about hyperbolic metric spaces Gro85, Gd90, $\mathrm{ABC}^{+} 91$. In this subsection we collect together some of these to fix notions and for later use.

If $X$ is a geodesic metric space and $x, y \in X$ then $[x, y]$ will denote a geodesic segment joining $x$ to $y$. For $x, y, z \in X$ we shall denote by $\triangle x y z$ a geodesic triangle with vertices $x, y, z$. For $D \geq 0$ and $A \subset X, N_{D}(A):=\{x \in X: d(x, a) \leq$ $D$ for some $a \in A\}$ will be called the $D$-neighborhood of $A$ in $X$.

Definition 1.22. Suppose $\Delta x_{1} x_{2} x_{3} \subset X$ is a geodesic triangle, and let $\delta \geq 0$, $K \geq 0$.

(1) For all $i \neq j \neq k \neq i$, let $c_{k} \in\left[x_{i}, x_{j}\right]$ be such that $d\left(x_{i}, c_{j}\right)=d\left(x_{i}, c_{k}\right)$. The points $c_{i}$ will be called the internal points of $\Delta x_{1} x_{2} x_{3}$. Note that, for all $i \neq j \neq k \neq i, d\left(x_{i}, c_{j}\right)=\frac{1}{2}\left\{d\left(x_{i}, x_{j}\right)+d\left(x_{i}, x_{k}\right)-d\left(x_{j}, x_{k}\right)\right\}$.

(2) The diameter of the set $\left\{c_{1}, c_{2}, c_{3}\right\}$ will be referred to as the insize of the triangle $\Delta x_{1} x_{2} x_{3}$.

(3) We say that the triangle $\Delta x_{1} x_{2} x_{3}$ is $\delta$-slim if any side of the triangle is contained in the $\delta$-neighborhood of the union of the other two sides.

(4) We say that the triangle $\Delta x_{1} x_{2} x_{3}$ is $\delta$-thin if for all $i \neq j \neq k \neq i$ and $p \in\left[x_{i}, c_{j}\right] \subset\left[x_{i}, x_{k}\right], q \in\left[x_{i}, c_{k}\right] \subset\left[x_{i}, x_{j}\right]$ with $d\left(p, x_{i}\right)=d\left(q, x_{i}\right)$ one has $d(p, q) \leq \delta$.

(5) A point $x \in X$ is said to be a $K$-center of $\triangle x_{1} x_{2} x_{3}$ if $x$ is contained in the $K$-neighborhood of each of the sides of $\triangle x_{1} x_{2} x_{3}$.

Definition 1.23. Gromov inner product: Let $X$ be any metric space and let $x, y, z \in X$. Then the Gromov inner product of $y, z$ with respect to $x$, denoted $(y . z)_{x}$, is defined to be the number $\frac{1}{2}\{d(x, y)+d(x, z)-d(y, z)\}$.

Definition 1.24. Let $\delta \geq 0$ and $X$ be a geodesic metric space. We say that $X$ is a $\delta$-hyperbolic metric space if all geodesic triangles in $X$ are $\delta$-slim.

Lemma 1.25. (See Proposition 2.1, $\left.\mathrm{ABC}^{+} 91\right]$ ) Suppose $X$ is a $\delta$-hyperbolic metric space. Then the following hold:

(1) All the triangles in $X$ have insize at most $4 \delta$.

(2) All the triangles in $X$ are $6 \delta$-thin.

Lemma 1.26. Gd90. Stability of quasigeodesics: For all $\delta \geq 0$ and $k \geq 1$ there is a constant $D \overline{1.26}=D \overline{1.26}(\delta, k)$ such that the following holds:

Suppose $Y$ is a $\delta$-hyperbolic metric space. Then the Hausdorff distance between a geodesic and a $k$-quasi-geodesic joining the same pair of end points is less than or equal to $D \overline{1.26}$.

Definition 1.27. Local quasi-geodesics: Let $X$ be a metric space and $K \geq$ $1, \epsilon \geq 0, L>0$ be constants. A map $f: I \rightarrow X$, where $I \subset \mathbb{R}$ is an interval, is said to be a $(K, \epsilon, L)$ - local quasi-geodesic if for all $s, t \in I$ with $|s-t| \leq L$, one has $-\epsilon+(1 / K)|s-t| \leq d(f(s), f(t)) \leq \epsilon+K|s-t|$.

For the following important lemma we refer to Theorem 1.4, Chapter 3, CDP90; or Theorem 21, Chapter 5, Gd90.

Lemma 1.28. Local quasi-geodesic vs global quasi-geodesic: For all $\delta \geq$ $0, \epsilon \geq 0$ and $K \geq 1$ there are constants $L=L \overline{1.28}(\delta, K, \epsilon), \lambda=\sqrt{1.28}(\delta, K, \epsilon)$ such that the following holds: 
Suppose $X$ is a $\delta$-hyperbolic metric space. Then any $(K, \epsilon, L)$-local quasi-geodesic in $X$ is a $\lambda$-quasi-geodesic.

Lemma 1.29. For all $\delta \geq 0, \epsilon \geq 0$ and $k \geq 1$, there is a constant D $1.29=$ $D \overline{1.29}(\delta, k, \epsilon)$ such that the following hold:

(1) Suppose $Y$ is a $\delta$-hyperbolic metric space. Then every geodesic triangle in $Y$ has a $4 \delta$-center.

(2) Suppose both $Y$ and $Y^{\prime}$ are $\delta$-hyperbolic metric spaces and $\phi: Y \rightarrow Y^{\prime}$ is a $(k, \epsilon)$-quasi-isometric embedding. If $y$ is a $4 \delta$-center of $\triangle y_{1} y_{2} y_{3} \subseteq Y$ and $y^{\prime} \in Y^{\prime}$ is a $4 \delta$-center of $\triangle \phi\left(y_{1}\right) \phi\left(y_{2}\right) \phi\left(y_{3}\right) \subseteq Y^{\prime}$ then $d\left(y^{\prime}, \phi(y)\right) \leq D \overline{1.29}$, where $d$ is the metric on $Y^{\prime}$.

Proof. By conclusion (1) of Lemma 1.25 the internal points of $\triangle y_{1} y_{2} y_{3}$ are $4 \delta$ centers of $\triangle y_{1} y_{2} y_{3}$. This proves part (1) of the lemma.

For (2), first we make the following observation: Let $\left\{c_{i}^{\prime}\right\}$ be the internal points of $\triangle \phi\left(y_{1}\right) \phi\left(y_{2}\right) \phi\left(y_{3}\right)$. Suppose $z \in Y^{\prime}$ is contained in a $D$-neighborhood of each of the sides of $\triangle \phi\left(y_{1}\right) \phi\left(y_{2}\right) \phi\left(y_{3}\right)$, for some $D \geq 0$. Let $p_{i} \in\left[\phi\left(y_{j}\right), \phi\left(y_{k}\right)\right], i \neq j \neq k \neq i$, be such that $d\left(p_{i}, z\right) \leq D, 1 \leq i, j, k \leq 3$; then $d\left(p_{i}, p_{j}\right) \leq 2 D$.

Claim: $d\left(c_{i}^{\prime}, p_{i}\right) \leq 3 D, i=1,2,3$.

Since the proofs are quite similar, we do the computation for $i=3$ for concreteness. Set $A_{i}=\phi\left(y_{i}\right), i=1,2,3$. Then

$$
\begin{aligned}
& 2 d\left(p_{3}, c_{3}^{\prime}\right) \\
& =2\left|d\left(A_{1}, c_{3}^{\prime}\right)-d\left(A_{1}, p_{3}\right)\right| \\
& =2\left|\left(A_{2}, A_{3}\right) A_{1}-d\left(A_{1}, p_{3}\right)\right| \\
& =\left|d\left(A_{1}, A_{3}\right)+d\left(A_{1}, A_{2}\right)-d\left(A_{2}, A_{3}\right)-2 d\left(A_{1}, p_{3}\right)\right| \\
& =\mid\left\{d\left(A_{1}, p_{2}\right)+d\left(A_{3}, p_{2}\right)\right\}+\left\{d\left(A_{1}, p_{3}\right)+d\left(A_{2}, p_{3}\right)\right\} \\
& \quad \quad-\left\{d\left(A_{3}, p_{1}\right)+d\left(A_{2}, p_{1}\right)\right\}-2 d\left(A_{1}, p_{3}\right) \mid \\
& =\left|\left\{d\left(A_{1}, p_{2}\right)-d\left(A_{1}, p_{3}\right)\right\}+\left\{d\left(A_{3}, p_{2}\right)-d\left(A_{3}, p_{1}\right)\right\}+\left\{d\left(A_{2}, p_{3}\right)-d\left(A_{2}, p_{1}\right)\right\}\right| \\
& \leq\left|d\left(A_{1}, p_{2}\right)-d\left(A_{1}, p_{3}\right)\right|+\left|d\left(A_{3}, p_{2}\right)-d\left(A_{3}, p_{1}\right)\right|+\left|d\left(A_{2}, p_{3}\right)-d\left(A_{2}, p_{1}\right)\right| \\
& \leq d\left(p_{2}, p_{3}\right)+d\left(p_{2}, p_{1}\right)+d\left(p_{3}, p_{1}\right) \\
& \leq 6 D
\end{aligned}
$$

This proves the claim and thus $d\left(z, c_{i}^{\prime}\right) \leq 4 D$ for all $i, 1 \leq i \leq 3$.

Since $\phi$ is a $(k, \epsilon)$-quasi-isometric embedding, it follows that $\phi(y)$ is contained in the $(4 k \delta+\epsilon)$ - neighborhood of the image under $\phi$ of each of the sides $\left[y_{i}, y_{j}\right]$, $i \neq j$. Also, the image of $\left[y_{i}, y_{j}\right]$, for all $i \neq j$, is a $(k, \epsilon)$-quasi-geodesic, and hence a $(k+\epsilon)$-quasi-geodesic, joining $\phi\left(y_{i}\right), \phi\left(y_{j}\right)$. By Lemma 1.26, $\phi(y)$ is contained in a $\{(4 k \delta+\epsilon)+D \overline{1.26}(\delta, k+\epsilon)\}-$ neighborhood of each of the sides of $\triangle \phi\left(y_{1}\right) \phi\left(y_{2}\right) \phi\left(y_{3}\right)$. Taking $D \overline{1.29}(\delta, k, \epsilon):=4 .\{(4 k \delta+\epsilon)+D \overline{1.26}(\delta, k+\epsilon)\}$, we are through.

Definition 1.30. Let $X$ be a geodesic metric space and let $A \subseteq X$. For $K \geq 0$, we say that $A$ is $K$-quasiconvex in $X$ if any geodesic with end points in $A$ is contained in the $K$-neighborhood of $A$. A subset $A \subset X$ is said to be quasi-convex if it is $K$-quasi-convex for some $K$.

Lemma 1.31. Let $X$ be a geodesic metric space.

(1) Let $p, q, r \in X$. Suppose $q$ is a nearest point projection of $p$ on a geodesic $[q, r]$ joining $q, r$. Then the arc length parametrization of the union 
$[p, q] \cup[q, r]$ is a $(3,0)$-quasi-geodesic in $X$.

(2) Suppose $U \subset X$ is a $K$-quasi-convex set and $p \notin U$. Suppose $q \in U$ is a nearest point projection of $p$ on $U$. Let $r \in U$. Then the arc length parametrization of the union $[p, q] \cup[q, r]$ is $(3+2 K)$-quasi-geodesic in $X$.

Proof. 1. Suppose $p_{1} \in[p, q], r_{1} \in[q, r]$. Then $q$ is a nearest point projection of $p_{1}$ on $\left[q, r_{1}\right]$. Thus $d\left(p_{1}, q\right) \leq d\left(p_{1}, r_{1}\right)$. Using the triangle inequality, $d\left(q, r_{1}\right) \leq$ $d\left(p_{1}, r_{1}\right)+d\left(p_{1}, q\right) \leq 2 d\left(p_{1}, r_{1}\right)$. Hence $d\left(p_{1}, q\right)+d\left(q, r_{1}\right) \leq 3 d\left(p_{1}, r_{1}\right)$.

2. Let $p_{1} \in[p, q], r_{1} \in[q, r]$. There exists $s \in U$ such that $d\left(r_{1}, s\right) \leq K$, since $U \subset X$ is $K$-quasi-convex. Now, as before, $q$ is a nearest point projection of $p_{1}$ on $U$. Hence $d\left(p_{1}, q\right) \leq d\left(p_{1}, s\right) \leq d\left(p_{1}, r_{1}\right)+K$ and so $d\left(q, r_{1}\right) \leq d\left(p_{1}, q\right)+d\left(p_{1}, r_{1}\right) \leq$ $2 d\left(p_{1}, r_{1}\right)+K$. Thus $d\left(p_{1}, q\right)+d\left(q, r_{1}\right) \leq 3 . d\left(p_{1}, r_{1}\right)+K$.

Lemma 1.32. For each $\delta \geq 0$ and $K \geq 0$ there is a constant $D \overline{1.32}=D \overline{1.32}(\delta, K)$ such that the following holds:

Suppose $X$ is a $\delta$-hyperbolic metric space and $V \subseteq U$ are $K$-quasi-convex subsets of $X$. Let $x \in X$ and let $x_{1}, x_{2}$ be nearest point projections of $x$ on $U$ and $V$ respectively. If $x_{3}$ is a nearest point projection of $x_{1}$ on $V$, then $d\left(x_{2}, x_{3}\right) \leq D_{1.32}$.

Proof. By Lemma $1.31(2),\left[x, x_{1}\right] \cup\left[x_{1}, x_{2}\right]$ is a $(3+2 k)$-quasi-geodesic. Hence by Lemma 1.26, there is a point $x_{4} \in\left[x, x_{2}\right]$ with $d\left(x_{1}, x_{4}\right) \leq D \overline{1.26}(\delta, 3+2 K)=D$, say. Similarly, $\left[x_{1}, x_{3}\right] \cup\left[x_{3}, x_{2}\right]$ is a $(3+2 K)$-quasi-geodesic and thus there is a point $x_{3}^{\prime} \in\left[x_{1}, x_{2}\right]$ such that $d\left(x_{3}, x_{3}^{\prime}\right) \leq D$. Using the $\delta$-slimness of $\triangle x_{1} x_{2} x_{4}$, there exists $x_{3}^{\prime \prime} \in\left[x_{2}, x_{4}\right]$ such that $d\left(x_{3}^{\prime}, x_{3}^{\prime \prime}\right) \leq D+\delta$. Hence $d\left(x_{3}, x_{3}^{\prime \prime}\right) \leq 2 D+\delta$. Since $x_{2}$ is a nearest point projection of $x_{3}^{\prime \prime}$ on $V$, we have $d\left(x_{2}, x_{3}^{\prime \prime}\right) \leq 2 D+\delta$. Thus $d\left(x_{2}, x_{3}\right) \leq d\left(x_{2}, x_{3}^{\prime \prime}\right)+d\left(x_{3}^{\prime \prime}, x_{3}\right) \leq 4 D+2 \delta$. Setting $D \overline{1.32}=4 D+2 \delta$ completes the proof of the lemma.

Definition 1.33. Suppose $Y$ is a metric space and $U, V \subset Y$. We say that $U, V$ are $\epsilon$-separated if $\inf \left\{d\left(y_{1}, y_{2}\right): y_{1} \in U, y_{2} \in V\right\} \geq \epsilon$. A collection of subsets $\left\{U_{\alpha}\right\}$ of $Y$ is said to be uniformly separated if there exists an $\epsilon>0$ such that any pair of distinct elements of the collection $\left\{U_{\alpha}\right\}$ is $\epsilon$-separated.

Definition 1.34. Suppose $Y$ is a $\delta$-hyperbolic metric space and $U_{1}, U_{2}$ are two quasi-convex subsets. Let $D>0$. We say that $U_{1}, U_{2}$ are mutually $D$-cobounded, or simply $D$-cobounded, if any nearest point projection of $U_{1}$ to $U_{2}$ has diameter at most $D$ and vice versa.

Lemma 1.35. Given $\delta \geq 0$ and $K \geq 0$ there are constants $R=R_{1.35}(\delta, K)$ and $D=D \overline{1.35}(\delta, K)$ such that the following holds:

Suppose $X$ is a $\delta$-hyperbolic metric space and $U, V \subset X$ are two $K$-quasiconvex and $R$-separated subsets. Then $U, V$ are $D$-cobounded.

Proof. Let $V_{1}(\subset V)$ be the set of all nearest point projections from points of $U$ to $V$. We want to show that $V_{1}$ is a set of uniformly bounded diameter for large enough $R$.

Suppose that $x_{1}, x_{2} \in U$ and let $y_{1}, y_{2} \in V$ be respectively their nearest point projections. Then by Lemma $1.31(2),\left[x_{1}, y_{1}\right] \cup\left[y_{1}, y_{2}\right]$ and $\left[x_{2}, y_{2}\right] \cup\left[y_{2}, y_{1}\right]$ are $K_{1}-$ quasi-geodesics for $K_{1}=(3+2 K)$. If $d\left(y_{1}, y_{2}\right) \geq D_{1}:=L \overline{1.28}\left(\delta, K_{1}, K_{1}\right)$ then the curve $\left[x_{1}, y_{1}\right] \cup\left[y_{1}, y_{2}\right] \cup\left[y_{2}, x_{2}\right]$ is a $\lambda={ }_{1.28}\left(\delta, K_{1}, K_{1}\right)$-quasi-geodesic by Lemma 
1.28. Hence every point of this curve is within distance $D \overline{1.26}(\delta, \lambda)+K$ from a point in $U$. Choosing $R=D \overline{1.26}(\delta, \lambda)+K+1$ proves the Lemma.

Lemma 1.36. Given $\delta \geq 0$ and $K \geq 0$ there are constants $R=R_{1.36}(\delta, K)$ and $D=D \overline{1.36}(\delta, K)$ such that the following holds:

Suppose $X$ is a $\delta$-hyperbolic metric space and $U, V \subset X$ are two $K$-quasiconvex and $R$-separated subsets. Then there are points $x_{0} \in U, y_{0} \in V$ such that $\left[x_{0}, y_{0}\right] \subset$ $N_{D}([x, y])$, for all $x \in U$ and $y \in V$.

Proof. First consider the set $V_{1}(\subset V)$ of all nearest point projections from points of $U$ onto $V$. By Lemma 1.35, there exists $R(=R[1.35)$ such that the diameter of $V_{1}$ is less than $D=D \overline{1.35}$ whenever $U, V$ are $R$-separated.

Choose any point $y_{0} \in V_{1}$ and let $x_{0}$ be a nearest point projection of $y_{0}$ onto $U$. Let $x \in U, y \in V$ be any pair of points and let $y_{1}$ be a nearest point projection of $x$ onto $V$. Since $y_{1} \in V_{1}$, it follows that $d\left(y_{0}, y_{1}\right) \leq D_{1}$. By Lemma 1.31 (2) $\left[y_{0}, x_{0}\right] \cup\left[x_{0}, x\right]$ is a $(3+2 K)$-quasi-geodesic. Since $X$ is a $\delta$-hyperbolic metric space, the Hausdorff distance between this quasi-geodesic and the geodesic $\left[x_{0}, x\right]$ is at most $D \overline{1.26}(\delta, 3+2 K)$ by Lemma 1.26 . Similarly the Hausdorff distance between $\left[x, y_{1}\right] \cup\left[y_{1}, y\right]$ and $[x, y]$ is at most $D_{1.26}(\delta, 3+2 K)$. Lastly, since $d\left(y_{0}, y_{1}\right) \leq D_{1}$, it follows that the Hausdorff distance between $\left[y_{0}, x\right]$ and $\left[y_{1}, x\right]$ is at most $\delta+D_{1}$. The Lemma follows by choosing $D=2 D \overline{1.26}(\delta, 3+2 K)+D_{1}+\delta$.

The following is a direct consequence of the proofs of Lemmas 1.35 and 1.36 (cf. Lemma 3.3 of [Mit98b]).

Corollary 1.37. Given $\delta \geq 0$ and $D, K \geq 0$ there exists $C=C \overline{1.37}(\delta, D, K)$ such that the following holds.

Suppose $X$ is a $\delta$-hyperbolic metric space and $U, V \subset X$ are two $K$-quasiconvex and $D$-cobounded subsets. Choose $a \in U, b \in V$ such that $d(a, b)=d(U, V)$, and $[c, a] \subset$ $U,[b, d] \subset V$ are $K$-quasigeodesics, then $[c, a] \cup[a, b] \cup[b, d]$ is a $C$-quasigeodesic.

The following Lemma Mit98b] says that quasi-isometries and nearest point projections 'almost commute'. We include a proof for completeness.

Lemma 1.38. (Lemma 3.5 of [Mit98b]) For all $\delta \geq 0$ and $k \geq 1$ there is a constant $D_{1.38}=D_{1.38}(\delta, k)$ such that the following holds:

Suppose $\phi: X \rightarrow Y$ is a $k$-quasi isometric embedding of $\delta$-hyperbolic metric spaces. Let $x, y, z \in X$ and let $\gamma$ be a geodesic in $X$ joining $x, y$. Let $u$ be a nearest point projection of $z$ onto $\gamma$ and suppose $v$ is a nearest point projection of $\phi(z)$ onto a geodesic joining $\phi(x)$ and $\phi(y)$, then $d(v, \phi(u)) \leq D \overline{1.38 \text {. }}$

Proof. Let $\left\{c_{i}\right\}$ and $\left\{c_{i}^{\prime}\right\}$ be respectively the internal points of $\triangle x y z \subset X$ and $\triangle \phi(x) \phi(y) \phi(z)$. By Lemma 1.31 (1) the unions $[x, u] \cup[u, z]$ and $[y, u] \cup[u, z]$ are both $(3,0)$-quasi-geodesics in $X$. It follows that they are 3-quasi-geodesics. Hence $u$ is contained in the $D \overline{1.26}(\delta, 3)$-neighborhood of both $[x, z]$ and $[y, z]$. Similarly, $v$ is contained in the $D \frac{1.26}{1.2}(\delta, 3)$-neighborhood of both $[\phi(x), \phi(z)]$ and $[\phi(y), \phi(z)]$. Therefore, using the proof of the claim in the proof of the Lemma 1.29) (2), we have $d_{X}\left(u, c_{i}\right) \leq 4 . P_{1.26}(\delta, 3)$ and $d_{Y}\left(v, c_{i}^{\prime}\right) \leq 4.2 \overline{1.26}(\delta, 3)$, for $i=1,2,3$.

Now for each $i, 1 \leq i \leq 3$ we have the following:

Since $\phi$ is a $k$-quasi-isometric embedding, we have $d_{Y}\left(\phi\left(c_{i}\right), c_{i}^{\prime}\right) \leq D \overline{1.29}(\delta, k, k)$ by Lemma 1.29(2). Thus, $d_{Y}\left(\phi\left(c_{i}\right), v\right) \leq d_{Y}\left(\phi\left(c_{i}\right), c_{i}^{\prime}\right)+d_{Y}\left(c_{i}^{\prime}, v\right) \leq D \overline{1.26}(\delta, 3)+$ $D \overline{1.29}(\delta, k, k)$. Again, using the fact that $\phi$ is a $k$-quasi-isometric embedding we 
have $d\left(\phi\left(c_{i}\right), \phi(u)\right) \leq k \cdot d_{X}\left(c_{i}, u\right)+k \leq k \cdot D_{1.26}(\delta, 3)+k$. Thus $d_{Y}(\phi(u), v) \leq$ $d_{Y}\left(\phi(u), \phi\left(c_{i}\right)\right)+d_{Y}\left(\phi\left(c_{i}\right), v\right) \leq k+(k+1) \cdot D_{1.26}(\delta, 3)+D_{1.29}(\delta, k, k)$. Choosing $D_{1.38}=k+(k+1) \cdot D_{1.26}(\delta, 3)+D \overline{1.29}(\delta, k, k)$ completes the proof.

To prove our main theorem, the following characterization of hyperbolicity turns out to be very useful.

Lemma 1.39. (Proposition 3.5 of [Ham07]) Suppose $X$ is a geodesic metric space and there is a collection of rectifiable curves $\{c(x, y): x, y \in X\}$, one for each pair of distinct points $x, y \in X$, and constants $D_{1}, D_{2} \geq 1$ such that for all $x, y, z \in X$ the following hold:

(1) If $d(x, y) \leq D_{1}$ then the length of the curve $c(x, y)$ is less than or equal to $D_{2}$.

(2) If $x^{\prime}, y^{\prime} \in c(x, y)$ then the Hausdorff distance between $c\left(x^{\prime}, y^{\prime}\right)$ and the segment of $c(x, y)$ between $x^{\prime}$ and $y^{\prime}$ is bounded by $D_{2}$.

(3) The triangle formed by the curves joining any three points in $X$ is $D_{2}$-slim: $c(x, y) \subseteq N_{D_{2}}(c(x, z) \bigcup c(y, z))$.

Then $X$ is $\overline{q_{1.39}}=\bar{q} \overline{1.39}_{(}\left(D_{1}, D_{2}\right)$-hyperbolic and each of the curves $c(x, y)$ is a $K_{[1.39]}=K_{[1.39}\left(D_{1}, D_{2}\right)$-quasi-geodesic in $X$.

This lemma has the following straightforward corollary, which is a discrete version of Lemma 1.39 (see Lemma 1.17 for instance). A discrete path $c(x, y)$ will refer to a finite sequence of points. The length of a discrete path is the sum of the distances between all pairs of successive points in the discrete path. In this context, a triangle will refer to the union of three discrete paths of the form $c(x, y), c(y, z)$, $c(z, x)$.

Corollary 1.40. Given $D, C_{1}, C_{2}>0$ and $\Phi: \mathbb{R}^{+} \rightarrow \mathbb{R}^{+}$, there exist $\dot{q 1.40}_{1 .}=$ $\Phi_{1.40}\left(D, C_{1}, C_{2}, \Phi\right) \geq 0$ and $K_{[1.40]}=K_{[1.40}\left(D, C_{1}, C_{2}, \Phi\right) \geq 1$ such that the following hold:

Let $X$ be a geodesic metric space and let $X_{1} \subset X$ be a discrete set such that $X=N_{D}\left(X_{1}\right)$.

Further suppose that for all $x \neq y \in X_{1}$, there is a discrete path $c(x, y)$ in $X_{1}$ connecting $x, y$ such that:

(1) Distance between successive points of $c(x, y)$ is at most $C_{1}$.

(2) If $d(x, y) \leq N$ then the number of points on the discrete path $c(x, y)$ is at most $f(N)$.

(3) If $x_{1} \neq y_{1}$ are two points of the discrete path $c(x, y)$, then the Hausdorff distance between the discrete path $c\left(x_{1}, y_{1}\right)$ and the discrete subpath of $c(x, y)$ connecting $x_{1}, y_{1}$ is at most $C_{2}$.

(4) For any three points $x, y, z \in X_{1}$, the triangle formed by the paths $c(x, y), c(y, z)$ and $c(x, z)$ is $C_{2}$-slim.

Then $X$ is $\delta_{1.40}$-hyperbolic and the discrete paths are $K_{1.40}$ quasi-geodesics in $X$.

Proof. Let $\phi: X \rightarrow X_{1}$ be a map such that for all $x \in X, d(x, \phi(x)) \leq D$.

Given $x, y \in X$ define a curve $\beta(x, y)$ joining $x, y$ as follows: Let $\phi(x)=$ $v_{1}, v_{2}, \ldots, v_{n}=\phi(y)$ be the set of successive points on $c(\phi(x), \phi(y))$. Join $x$ to $\phi(x), v_{i}$ to $v_{i+1}$, for $1 \leq i \leq n-1$, and $\phi(y)$ to $y$ by geodesics in $X$ to obtain $\beta(x, y)$.

We check that the curves $\{\beta(x, y)\}$ satisfy the conditions of Lemma 1.39 
(1) That the paths $\beta(x, y)$ are rectifiable follows from conditions 1 and 2.

(2) We verify that condition 1 of Lemma 1.39 is satisfied with $D_{1}=1$. Let $x, y \in X$ such that $d(x, y) \leq 1$. Then $d(\phi(x), \phi(y)) \leq d(\phi(x), x)+d(x, y)+$ $d(y, \phi(y)) \leq 2 D+1$. Hence there are at most $\Phi(2 D+1)$ points on the discrete path $c(\phi(x), \phi(y))$. Let $\phi(x)=v_{1}, v_{2}, \ldots, v_{n}=\phi(y)$ be the set of successive points on $c(\phi(x), \phi(y))$. Then $n \leq \Phi(2 D+1)$ and hence the length of the path $\beta(x, y)=d(x, \phi(x))+d(y, \phi(y))+\sum_{i=1}^{n} d\left(v_{i}, v_{i+1}\right) \leq$ $2 D+\Phi(2 D+1) C_{1}$. Thus we may choose $D_{2} \geq 2 D+\Phi(2 D+1) C_{1}$.

(3) Conditions 2 and 3 of Lemma 1.39 follow from conditions 3,4. In fact, choosing $D_{2} \geq C_{2}+2 C_{1}$ is enough for this.

Hence, choosing $D_{2}=\max \left\{2 D+\Phi(2 D+1) C_{1}, C_{2}+2 C_{1}\right\}$ completes the proof.

1.3. Trees of hyperbolic and relatively hyperbolic metric spaces. We refer to Far98 for a detailed account of relative hyperbolicity. We also refer to MR08 for the definitions and results of this subsection.

Suppose $(X, d)$ is a path metric space and let $\mathcal{H}=\left\{H_{\alpha}\right\}$ be a collection of path-connected, uniformly separated subsets of $X$. Then Farb Far98 defines the electric space (or coned-off space) $\mathcal{E}(X, \mathcal{H})$ corresponding to the pair $(X, \mathcal{H})$ as a metric space which consists of $X$ and a collection of vertices $v_{\alpha}$ (one for each $H_{\alpha} \in \mathcal{H}$ ) such that each point of $H_{\alpha}$ is joined to (equivalently, coned off at) $v_{\alpha}$ by an edge of length $\frac{1}{2}$. The sets $H_{\alpha}$ shall be referred to as horosphere-like sets and the vertices $v_{\alpha}$ as cone-points. Geodesics (resp. $P$-quasigeodesics) in $\mathcal{E}(X, \mathcal{H})$ will be called electric geodesics (resp. electric P-quasigeodesics).

When the collection $\mathcal{H}=\left\{H_{\alpha}\right\}$ is not necessarily separated, a slightly modified description is given in Mj06 and MR08, where we attach a metric product $H_{\alpha} \times$ $[0,1]$ to $X$, identifying $H_{\alpha} \times\{0\}$ with $H_{\alpha} \subset X$ for each $H_{\alpha} \in \mathcal{H}$, and equip each $H_{\alpha} \times\{1\}$ with the zero metric. We shall call this construction electrocution.

Let $i: X \rightarrow \mathcal{E}(X, \mathcal{H})$ denote the natural inclusion of spaces. Then for a path $\gamma \subset X$, the path $i(\gamma)$ lies in $\mathcal{E}(X, \mathcal{H})$. Replacing maximal subsegments $[a, b]$ of $i(\gamma)$ lying in a particular $H_{\alpha}$ by a path that goes from $a$ to $v_{\alpha}$ and then from $v_{\alpha}$ to $b$, and repeating this for every $H_{\alpha}$ that $i(\gamma)$ meets we obtain a new path $\hat{\gamma}$. If $\hat{\gamma}$ is an electric geodesic (resp. electric $P$-quasigeodesic), $\gamma$ is called a relative geodesic (resp. relative $P$-quasigeodesic). A geodesic, or quasigeodesic, or more generally a path $\gamma$ is said to be without backtracking if for any horosphere-like set $H_{\alpha}, \gamma$ does not return to $H_{\alpha}$ after leaving it.

Definition 1.41. Far98 Relative $P$-quasigeodesics in $(X, \mathcal{H})$ are said to satisfy bounded region penetration if, for any two relative $P$-quasigeodesics without backtracking $\beta, \gamma$, joining $x, y \in X$, there exists $B=B(P) \geq 0$ such that

Similar Intersection Patterns 1: if precisely one of $\{\beta, \gamma\}$ meets a horospherelike set $H_{\alpha}$, then the length (measured in the intrinsic path-metric on $H_{\alpha}$ ) from the first (entry) point to the last (exit) point (of the relevant path) is at most $B$.

Similar Intersection Patterns 2: if both $\{\beta, \gamma\}$ meet some $H_{\alpha}$ then the length (measured in the intrinsic path-metric on $H_{\alpha}$ ) from the entry point of $\beta$ to that of $\gamma$ is at most $B$; similarly for exit points.

$X$ is strongly hyperbolic relative to the collection $\mathcal{H}$ if

a) $\mathcal{E}(X, \mathcal{H})$ is hyperbolic, and

b) Relative quasigeodesics satisfy the bounded region penetration property. 
The next notion is based on Bestvina-Feighn's seminal work BF92]. The notions we use here are the adaptations used in MR08.

Definition 1.42. A geodesic metric space $(X, d)$ equipped with a map $P: X \rightarrow T$ to a simplicial tree $T$ is said to be a tree of geodesic metric spaces satisfying the $q$ (uasi) i(sometrically) embedded condition if there exist $\epsilon \geq 0$ and $K \geq 1$ satisfying the following:

1) For all vertices $v \in T, X_{v}=P^{-1}(v) \subset X$ with the induced path metric $d_{X_{v}}$ is a geodesic metric space. Further, the inclusions $i_{v}: X_{v} \rightarrow X$ are uniformly proper.

2) Let $e$ be an edge of $T$ with initial and final vertices $v_{1}$ and $v_{2}$ respectively. Let $X_{e}$ be the pre-image under $P$ of the mid-point of e. There exist continuous maps $f_{e}: X_{e} \times[0,1] \rightarrow X$, such that $\left.f_{e}\right|_{X_{e} \times(0,1)}$ is an isometry onto the pre-image of the interior of e equipped with the path metric. Further, $f_{e}$ is fiber-preserving, i.e. projection to the second co-ordinate in $X_{e} \times[0,1]$ corresponds via $f_{e}$ to projection to the tree $P: X \rightarrow T$.

3) $\left.f_{e}\right|_{X_{e} \times\{0\}}$ and $\left.f_{e}\right|_{X_{e} \times\{1\}}$ are $(K, \epsilon)$-quasi-isometric embeddings into $X_{v_{1}}$ and $X_{v_{2}}$ respectively. $\left.f_{e}\right|_{X_{e} \times\{0\}}$ and $\left.f_{e}\right|_{X_{e} \times\{1\}}$ will occasionally be referred to as $f_{e, v_{1}}$ and $f_{e, v_{2}}$ respectively.

$X_{v}, X_{e}$ are referred to as vertex and edge spaces respectively. A tree of spaces as in Definition 1.42 above is said to be a tree of hyperbolic metric spaces, if there exists $\delta \geq 0$ such that $X_{v}, X_{e}$ are all $\delta$-hyperbolic for all vertices $v$ and edges $e$ of $T$.

Definition 1.43. A tree $P: X \rightarrow T$ of geodesic metric spaces is said to be a tree of relatively hyperbolic metric spaces if in addition to the conditions of Definition 1.42, we have the following:

4) Each vertex (or edge) space $X_{v}$ (or $X_{e}$ ) is strongly hyperbolic relative to a collection $\mathcal{H}_{v}\left(\right.$ or $\left.\mathcal{H}_{e}\right)$

5) the maps $f_{e, v_{i}}$ above $(i=1,2)$ are strictly type-preserving, i.e. $f_{e, v_{i}}^{-1}\left(H_{v_{i}, \alpha}\right)$, $i=1,2$ (for any $H_{v_{i}, \alpha} \in \mathcal{H}_{v_{i}}$ ) is either empty or some $H_{e, \beta} \in \mathcal{H}_{e}$. Also, for all $H_{e, \beta} \in \mathcal{H}_{e}$, and any end-point $v$ of $e$, there exists $H_{v, \alpha}$, such that $f_{e, v}\left(H_{e, \beta}\right) \subset H_{v, \alpha}$. The sets $H_{v, \alpha}$ and $H_{e, \alpha}$ will be referred to as horosphere-like vertex sets and horosphere-like edge sets respectively.

6) There exists $\delta>0$ such that each $\mathcal{E}\left(X_{v}, \mathcal{H}_{v}\right)$ is $\delta$-hyperbolic.

Given the tree of spaces with vertex spaces $X_{v}$ and edge spaces $X_{e}$, there exists a naturally associated tree of spaces with vertex spaces $\mathcal{E}\left(X_{v}, \mathcal{H}_{v}\right)$ and edge spaces $\mathcal{E}\left(X_{e}, \mathcal{H}_{e}\right)$, obtained by simply coning off the respective horosphere like sets. Condition (5) above ensures that we have natural inclusion maps of edge spaces $\mathcal{E}\left(X_{e}, \mathcal{H}_{e}\right) \times\{i\}(i=0,1)$ into adjacent vertex spaces $\mathcal{E}\left(X_{v}, \mathcal{H}_{v}\right)$. These maps are referred to as induced maps. The resulting tree of coned-off spaces will be called the induced tree of coned-off spaces and will be denoted as $\widehat{X}$.

7) The induced maps of the coned-off edge spaces into the coned-off vertex spaces $\widehat{f_{e, v_{i}}}: \mathcal{E}\left(X_{e}, \mathcal{H}_{e}\right) \rightarrow \mathcal{E}\left(X_{v_{i}}, \mathcal{H}_{v_{i}}\right) \quad(i=1,2)$ are uniform quasi-isometries. This is called the qi-preserving electrocution condition.

$d_{v}$ and $d_{e}$ will denote path metrics on $X_{v}$ and $X_{e}$ respectively. $i_{v}, i_{e}$ will denote inclusion of $X_{v}, X_{e}$ respectively into $X$.

Note that the first clause of Condition (5) above ensures that for any vertex $v_{i}$ and edge $e$ incident on $v_{i}$, and for any horosphere like set $H_{v_{i}, \alpha}$ in $X_{v_{i}}$, at most one horosphere like set $H_{e, \beta}$ of $X_{e}$ is mapped by $f_{e, v_{i}}$ into $H_{v_{i}, \alpha}$. Also, the second 
clause of Condition (5) above ensures that for any such horosphere like set $H_{e, \beta}$ of $X_{e}, f_{e, v_{i}}$ maps $H_{e, \beta}$ into some horosphere like set $H_{v_{i}, \alpha}$ in $X_{v_{i}}$.

Definition 1.44. The cone locus of the induced tree (T) of coned-off spaces, $\widehat{X}$, is the graph whose vertex set $\mathcal{V}$ consists of horosphere like vertex sets and edge set $\mathcal{E}$ consists of horosphere like edge sets such that an edge $H_{e, \beta} \in \mathcal{H}_{e} \subset \mathcal{E}$ is incident on a vertex $H_{v, \alpha} \in \mathcal{H}_{v} \subset \mathcal{V}$ iff $f_{e, v}\left(H_{e, \beta}\right) \subset H_{v, \alpha}$.

A connected component of the cone-locus will be called a maximal cone-subtree. The collection of maximal cone-subtrees will be denoted by $\mathcal{T}$ and elements of $\mathcal{T}$ will be denoted as $T_{\alpha}$.

For each maximal cone-subtree $T_{\alpha}$, we define the associated maximal cone-subtree of horosphere-like spaces $C_{\alpha}$ to be the tree of metric spaces whose vertex and edge spaces are the horosphere like vertex and edge sets $H_{v, \alpha}, H_{e, \alpha}, v \in \mathcal{V}\left(T_{\alpha}\right)$, $e \in \mathcal{E}\left(T_{\alpha}\right)$, along with the restrictions of the maps $f_{e}$ to $H_{e, \alpha} \times\{0,1\}$. The collection of $C_{\alpha}$ 's will be denoted as $\mathcal{C}$.

The next definition is based on BF92 again.

Definition 1.45. A disk $f:[-m, m] \times I \rightarrow X$ is a hallway of length $2 m$ if it satisfies:

1) $f^{-1}\left(\cup X_{e}: e \in \operatorname{Edge}(T)\right)=\{-m, \cdots, m\} \times I$, where $\operatorname{Edge}(T)$ denotes the collection of mid-points of the edge-set of $T$.

2) $f$ maps $i \times I$ to a geodesic in $X_{e}$ for some edge space.

3) $f$ is transverse, relative to condition (1), to $\cup_{e} X_{e}$, i.e. for all $i \in\{-m, \cdots, m\}$, $\left.f\right|_{B\left(i, \frac{1}{4}\right) \times\{t\}}$ is an isometric embedding for all $t \in I$. Here $B\left(i, \frac{1}{4}\right)$ denotes the $\frac{1}{4}$ neighborhood of $i$ in $[-m, m]$.

Condition (3) above is the adaptation in our context of Condition (2) of BF92 p.87 and simply says that a hallway transversely cuts across the collection of edge spaces.

Definition 1.46. A hallway $f:[-m, m] \times I \rightarrow X$ is $\rho$-thin if $d(f(i, t), f(i+1, t)) \leq$ $\rho$ for all $i \in\{-m, \cdots, m\}$ and $t \in I$.

A hallway is $\lambda$-hyperbolic if

$$
\lambda l(f(\{0\} \times I)) \leq \max \{l(f(\{-m\} \times I)), l(f(\{m\} \times I))\}
$$

where $l(\sigma)$ denotes the length of the path $\sigma$.

The girth of the hallway is the quantity $l(f(\{0\} \times I))$.

A hallway is essential if the edge path in $T$ resulting from projecting $f([-m, m] \times I)$ onto $T$ does not backtrack, and is therefore a geodesic segment in the tree $T$.

Hallways flare condition: The tree of spaces, $X$, is said to satisfy the hallways flare condition if there are numbers $\lambda>1$ and $m \geq 1$ such that for all $\rho$ there is a constant $H=H(\rho)$ such that any $\rho$-thin essential hallway of length $2 m$ and girth at least $H$ is $\lambda$-hyperbolic.

Definition 1.47. An essential hallway of length $2 \mathrm{~m}$ is cone-bounded if a) $f(i \times \partial I)=f(i \times\{0,1\})$ lies in the cone-locus for $i=\{-m, \cdots, m\}$. b) $f(i \times\{0\})$ and $f(i \times\{1\})$ lie in different components of the cone-locus.

The tree of spaces, $X$, is said to satisfy the cone-bounded hallways strictly flaring condition if for all $\rho>0$, there exists $\lambda>1$ and $m \geq 1$ such that any cone-bounded $\rho$-thin essential hallway of length $2 m$ is $\lambda$-hyperbolic. 
Note that the last condition requires all cone-bounded $\rho$-thin essential hallways to flare (not just those of girth at least $H$ as in Definition 1.46). The following theorem is one of the main results of MR08.

Theorem 1.48. MR08 Let $X$ be a tree $(T)$ of strongly relatively hyperbolic spaces satisfying

(1) the qi-embedded condition.

(2) the strictly type-preserving condition.

(3) the qi-preserving electrocution condition.

(4) the induced tree of coned-off spaces satisfies the hallways flare condition.

(5) the cone-bounded hallways strictly flaring condition.

Then $X$ is (strongly) hyperbolic relative to the family $\mathcal{C}$ of maximal cone-subtrees of horosphere-like spaces.

Note: In MR08 the definition of cone-bounded hallways does not include Condition (b) of Definition 1.47. However the proof there (cf. Proposition 4.4 of MR08) is enough to give Theorem 1.48 under the (weaker) condition that only those cone-bounded hallways (in the terminology of [MR08]) that additionally satisfy restriction (b) strictly flare.

Definition 1.49. Partial Electrocution: $\operatorname{Let}(X, \mathcal{H}, \mathcal{G}, \mathcal{L})$ be an ordered quadruple, where

(1) $X$ is a geodesic metric space,

(2) $\mathcal{H}=\left\{H_{\alpha}\right\}$ is a collection of uniformly separated subsets of $X$,

(3) $\mathcal{L}=\left\{L_{\alpha}\right\}$ is a collection of $\delta$ - hyperbolic metric spaces for some $\delta \geq 0$,

(4) $\mathcal{G}=\left\{g_{\alpha}: H_{\alpha} \rightarrow L_{\alpha}\right\}$ are maps.

Further suppose that there exist $K \geq 1$ such that the following hold:

(1) $X$ is strongly hyperbolic relative to the collection $\mathcal{H}$ of subsets $H_{\alpha}$.

(2) Each $g_{\alpha}$ is $K$ - coarsely Lipschitz, i.e. $d_{L_{\alpha}}\left(g_{\alpha}(x), g_{\alpha}(y)\right) \leq K d_{H_{\alpha}}(x, y)+K$ for all $x, y \in H_{\alpha}$.

The partially electrocuted space or partially coned off space corresponding to $(X, \mathcal{H}, \mathcal{G}, \mathcal{L})$ is the quotient metric space $\left(\hat{X}, d_{\text {pel }}\right)$ obtained from $X$ by attaching the metric mapping cylinders for the maps $g_{\alpha}: H_{\alpha} \rightarrow L_{\alpha}$, where $d_{\text {pel }}$ denotes the resulting partially electrocuted metric. (The metric mapping cylinder for a map $g: A \rightarrow B$ is the quotient metric space obtained as a quotient space of the disjoint union of the metric product $A \times[0,1]$ and $B$, by identifying $(a, 1) \in A \times\{1\}$ with $g(a) \in B$.)

Lemma 1.50. MR08 (see also Lemmas 1.20. 1.21 of MP11]) $\left(\hat{X}, d_{\text {pel }}\right)$ is a hyperbolic metric space and the sets $L_{\alpha}$ are uniformly quasiconvex in $\hat{X}$.

We end this subsection with a proposition, a special case of which is due to Hamenstadt Ham05], where the tree is taken to be $\mathbb{R}$ with vertex set $\mathbb{Z}$. We give a different proof below as our proof applies in a more general context.

Proposition 1.51. Given $K \geq 1$ and $\delta, D>0$, there exist $\delta^{\prime}, k^{\prime} \geq 0$ such that the following holds.

Suppose $Y$ is a tree of $\delta$-hyperbolic metric spaces satisfying the $K$-qi embedded condition such that the images of the edge spaces in the vertex spaces are mutually $D$-cobounded. Then $Y$ is a $\delta^{\prime}$-hyperbolic metric space and all the vertex spaces and edge spaces are $k^{\prime}$-quasiconvex in $Y$. 
Proof. First of all we note that by [Bow97] (Section 7, esp. Proposition 7.4, Lemma 7.5, Proposition 7.12; see also Lemma 3.4 of (Mj11) the vertex spaces are strongly hyperbolic relative to the images of edge spaces. Hence $Y$ can be thought of as a tree of relatively hyperbolic metric spaces whose horosphere like edge sets and vertex sets are respectively the whole of the edge spaces and the images of the edge spaces in the vertex spaces respectively. Hence conditions (1)-(2) of Theorem 1.48 are satisfied in this case.

Edge spaces after electrocution become points. Vertex spaces after electrocution become hyperbolic metric spaces. Inclusion of points into spaces being trivially qi-embeddings, condition (3) of Theorem 1.48 is satisfied.

Next any essential hallway of length greater than two in $\hat{Y}$, the induced tree of coned off spaces, must have girth at most one. This is because all edge spaces have diameter one after electrocution. Hence Condition (4) of Theorem 1.48 is trivially satisfied by choosing the threshold value $H$ of the girth to be 2 .

Finally, since cone-bounded hallways must have length two or more, it follows that in the present situation cone-bounded hallways do not exist due to Condition (b) in Definition 1.47 and the fact that entire edge spaces are part of the cone locus. Hence Condition (5) of Theorem 1.48 is vacuously satisfied.

Finally, the family $\mathcal{C}$ of maximal cone-subtrees of horosphere-like spaces are precisely the edge spaces.

Hence $Y$ is strongly hyperbolic relative to the edge spaces.

The edge spaces are uniformly hyperbolic with respect to the induced length metric from $Y$. Hence, by Lemma 1.50, we see that when the maps $g_{\alpha}$ are taken to be identity maps of the edge spaces, the partially electrocuted space is hyperbolic. This space is clearly quasi-isometric to $Y$. Hence the result.

As an application of this proposition we have the following corollary which can be thought of as a 'discrete' or 'graph' version of Proposition [1.51.

Corollary 1.52. Given $\delta, D, D_{1}, K \geq 1$, there exists $D \overline{1.52}$ such that the following holds.

Suppose $X$ is a connected graph and $X_{i}, 0 \leq i \leq n$, are connected subgraphs with $X=\cup_{i} X_{i}$ such that the following conditions hold.

(1) All the spaces $X_{i}$ are $\delta$-hyperbolic with respect to the path metric induced from $X$.

(2) $X_{i} \cap X_{j} \neq \emptyset$ iff $|i-j| \leq 1$.

(3) For all $i, X_{i} \cap X_{i+1}$ contains a connected subgraph $Y_{i}$ and is contained in the $D$-neighborhood of $Y_{i}$ in (the path-metric on) $X_{i}$ as well as $X_{i+1}$.

(4) The inclusions $Y_{i} \hookrightarrow X_{i}, Y_{i} \hookrightarrow X_{i+1}$ are $K$-quasi-isometric embeddings. Also the inclusions $Y_{i} \hookrightarrow X, 1 \leq i \leq n-1$ are uniformly metrically proper as measured by $g$, for some map $g: \mathbb{R}^{+} \rightarrow \mathbb{R}^{+}$.

(5) $Y_{i}$ and $Y_{i+1}$ are $D_{1}$-cobounded in $X_{i+1}$.

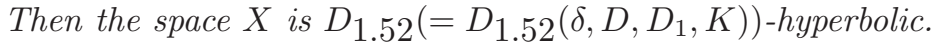

Proof. First construct a new graph $X^{\prime}$ with the same vertex set as $X$ and edge-set $\mathcal{E}\left(X^{\prime}\right)=\mathcal{E}(X) \bigcup\left\{\{u, v\}: u \neq v \in \mathcal{V}\left(X_{i}\right)\right.$ for some $\left.i ; d_{X}(u, v) \leq D\right\}$. Note that $X$ is a subgraph of $X^{\prime}$. By Lemma $1.17(2), X$ is quasi-isometric to $X^{\prime}$.

Let us denote by $X_{i}^{\prime}$ the subgraph of $X^{\prime}$ with the same vertex set as $X_{i}$ (i.e. $\left.\mathcal{V}\left(X_{i}^{\prime}\right)=\mathcal{V}\left(X_{i}\right)\right)$ and with edge-set $\mathcal{E}\left(X_{i}^{\prime}\right)=\left\{\{u, v\}: u \neq v \in \mathcal{V}\left(X_{i}\right) ; d_{X}(u, v) \leq\right.$ 
$D\}$. Then $X^{\prime}=\cup_{i} X_{i}^{\prime}$. Let $Y_{i}^{\prime}:=X_{i}^{\prime} \cap X_{i+1}^{\prime}$. Note that $Y_{i}^{\prime}$ is a connected graph by Condition (3).

We show now that $Y_{i}$ is quasi-isometric to $Y_{i}^{\prime}$. First, since the inclusion $Y_{i} \hookrightarrow X$ is uniformly proper, it follows that the inclusion $Y_{i} \hookrightarrow X^{\prime}$ is also uniformly proper (since $X$ is quasi-isometric to $X^{\prime}$ ). Hence the inclusion $Y_{i} \hookrightarrow Y_{i}^{\prime}$ is also uniformly proper. But the vertex set of $Y_{i}^{\prime}$ is contained in a $D$-neighborhood of $Y_{i}$ in $X$. Hence every vertex of $Y_{i}^{\prime}$ is connected by an edge to a vertex of $Y_{i}$ by construction of $X^{\prime}$. It follows that the inclusion $Y_{i} \hookrightarrow Y_{i}^{\prime}$ is a uniform (independent of $i$ ) quasi-isometry.

Next we claim that the inclusion of $X_{i}$ into $X_{i}^{\prime}$ is a uniform (independent of $i$ ) quasi-isometry. Note that $X_{i}$ and $X_{i}^{\prime}$ have the same vertex set. Also the inclusion $X_{i} \hookrightarrow X_{i}^{\prime}$ is 1-Lipschitz. Hence it suffices to show that when two vertices in $X_{i}$ are at a distance of at most $D$ in $X$ then they are not too far away in the (path) metric on $X_{i}$. Let $\gamma$ be a geodesic in $X$ joining two points of $X_{i}$ that are at a distance of at most $D$ from each other. If $\gamma$ contains a (maximal) subsegment $\gamma_{0}=\left[a_{0}, b_{0}\right]$ lying outside $X_{i}$ then $a_{0}, b_{0}$ must be distinct vertices of $Y_{i}^{\prime}$ or $Y_{i+1}^{\prime}$. Without loss of generality, suppose $a_{0}, b_{0} \in \mathcal{V}\left(Y_{i}^{\prime}\right)$. Hence there exist vertices $a, b \in \mathcal{V}\left(Y_{i}\right)$ such that $d_{X}\left(a, a_{0}\right) \leq D$ and $d_{X}\left(b, b_{0}\right) \leq D$. It follows that $d_{Y_{i}}(a, b) \leq g(3 D)$ and hence $d_{X_{i}}(a, b) \leq g(3 D)$. The claim follows.

Hence there exist $\delta^{\prime}, K^{\prime}, D_{1}^{\prime}$ such that $X_{i}^{\prime}$ is $\delta^{\prime}$ hyperbolic; the inclusion maps $Y_{i}^{\prime} \hookrightarrow X_{i}^{\prime}, Y_{i}^{\prime} \hookrightarrow X_{i+1}^{\prime}$ are $K^{\prime}$-qi embeddings; and $Y_{i}^{\prime}$ and $Y_{i+1}^{\prime}$ are $D_{1}^{\prime}$-cobounded in $X_{i+1}^{\prime}$ for all $i$.

Now we construct a tree of metric spaces $X_{T}$ quasi-isometric to $X^{\prime}$ (and hence to $X$ ) where the underlying tree $T$ is the interval $[0, n]$ with vertices the integer points $\{0, \cdots, n\}$ and edge set $\{[i, i+1]: i=0, \cdots, n-1\}$. For each $Y_{i}^{\prime}$ construct $Y_{i}^{\prime} \times[0,1] . X_{T}$ is constructed as an identification space from $\cup_{i}\left(Y_{i}^{\prime} \times[0,1]\right) \cup \cup_{i} X_{i}^{\prime}$ as follows. For all $i=0 \cdots n-1$ and $x \in \mathcal{V}\left(Y_{i}^{\prime}\right)$, identify $x \times\{0\}$ with $x \in X_{i}^{\prime}$ and $x \times\{1\}$ with $x \in X_{i+1}^{\prime}$. Extend the identification linearly over edges of $Y_{i}^{\prime}$ for all $i$ to obtain the required tree of metric spaces $X_{T}$. We observe that $X^{\prime}$ (and hence $X)$ is quasi-isometric to the tree of metric spaces $X_{T}$, which in turn satisfies all the conditions of Proposition 1.51 The Corollary follows.

A remark is in order here. Note that in the hypothesis we have not required that each $X_{i}$ contains all the edges of $X$ between any two of its vertices. But it is always true that $X_{i-1} \cup X_{i} \cup X_{i+1}$ contains all the edges of $X$ between any two vertices of $X_{i}$ since $X=\cup_{i} X_{i}$. However, once we pass to $X^{\prime}$ this is no longer an issue because in the construction of $X_{i}^{\prime}$ from $X_{i}$ these edges get introduced in any case (as $D \geq 1$ ). Hence each $X_{i}^{\prime}$ contains all the edges of $X^{\prime}$ between any two of its vertices.

\section{QI SECTIONS}

2.1. Existence of qi sections. The main result (Proposition 2.10) of this subsection is that qi sections exist for a large class of examples of metric graph bundles $p: X \rightarrow B$. This is the crucial ingredient in the proof of our main theorem 4.3 The basic idea of the proof of Proposition 2.10 runs as follows:

We assume that the horizontal spaces $F_{b}, b \in \mathcal{V}(B)$ in our metric graph bundle are uniformly hyperbolic and the barycenter maps $\phi_{b}: \partial^{3} F_{b} \rightarrow F_{b}$, sending a triple of distinct points on the boundary $\partial F_{b}$ to the barycenter of an ideal triangle with the 
three points as vertices, are uniformly coarsely surjective. For simplicity, suppose we have $x \in F_{v}, v \in B$ and there is a triple $\xi=\left(\xi_{1}, \xi_{2}, \xi_{3}\right)$ such that $\phi_{v}(\xi)=x$. Fix one such triple. 'Flow' this triple to the boundaries of all other horizontal spaces $F_{w}$ by maps induced by quasi-isometries $f_{v w}: F_{v} \rightarrow F_{w}$. These maps are coarsely unique and are naturally associated to any given metric graph bundle. Let $\partial\left(f_{v w}\right)$ denote the boundary value of $f_{v w}$. Consider the barycenters of the ideal triangles formed by the flowed triples $\left(\partial\left(f_{v w}\right) \xi_{1}, \partial\left(f_{v w}\right) \xi_{2}, \partial\left(f_{v w}\right) \xi_{3}\right)$. The collection of all these barycenters (as $w$ ranges over $\mathcal{V}(B)$ ) is then a section through $x$. The proof that this is indeed a qi section hinges on the fact that for any three points $u, v, w \in \mathcal{V}(B)$, the quasi-isometries $f_{u v}$ and $f_{w v} \circ f_{u w}$ are at a bounded distance (depending on $u, v, w$ ) from each other, and hence the induced boundary maps satisfy the equality $\partial\left(f_{u v}\right)=\partial\left(f_{w v}\right) \circ \partial\left(f_{u w}\right)$.

As an application of the proof of this result we recover an important lemma due to Mosher (see Theorem 2.11). It should be noted that though a basic ingredient for both Mosher's proof and ours is the notion of a 'barycenter', we do not have a group action on the boundaries of fiber spaces in our context. Mosher extracts his qi-section from an action of the whole group on the boundary of the normal subgroup.

Definition 2.1. Sequential Boundary (See Chapter $\left.4, \mathrm{ABC}^{+91}\right)$ Let $X$ be $a \delta$ hyperbolic metric space. A sequence of points $\left\{x_{n}\right\}$ in $X$ is said to converge to infinity, written $x_{n} \rightarrow \infty$, if for some (and hence any) point $p \in X, \lim _{m, n \rightarrow \infty}\left(x_{m} . x_{n}\right)_{p}=$ $\infty$.

Define an equivalence relation on the set of all sequences in $X$ converging to infinity, by setting $\left\{x_{n}\right\} \sim\left\{y_{n}\right\}$ iff $\lim _{n \rightarrow \infty}\left(x_{n} \cdot y_{n}\right)_{p}=\infty$. The set of all equivalence classes $\left\{\left[\left\{x_{n}\right\}\right]: x_{n} \rightarrow \infty\right\}$ will be denoted by $\partial X$ and will be referred to as the sequential boundary of $X$ or simply the boundary of $X$.

Suppose $\left\{x_{n}\right\}$ is a sequence of points in $X$ and $x_{n} \rightarrow \infty$. We shall write $x_{n} \rightarrow$ $\xi \in \partial X$ to mean that $\xi=\left[\left\{x_{n}\right\}\right]$. The boundary $\partial X$ comes equipped with a natural 'visual' topology Gd90].

Suppose $f: X \rightarrow Y$ is a $(k, \epsilon)$-quasi-isometric embedding of hyperbolic metric spaces and $\xi=\left[\left\{x_{n}\right\}\right] \in \partial X$. Then $f\left(x_{n}\right) \rightarrow \infty$. Setting $\partial(f)(\xi):=\left[\left\{f\left(x_{n}\right)\right\}\right]$ gives a well defined map $\partial(f): \partial X \rightarrow \partial Y$. The next lemma collects together standard properties of such maps.

Lemma 2.2. 1) If $I_{X}: X \rightarrow X$ is the identity map then $\partial\left(I_{X}\right)$ is the identity map on the sequential boundary of $X$.

2) If $f: X \rightarrow Y$ and $g: Y \rightarrow Z$ are two $(k, \epsilon)$-quasi-isometric embeddings then $\partial(g \circ f)=\partial(g) \circ \partial(f)$.

3) If $f, g: X \rightarrow Y$ are two $(k, \epsilon)$-quasi-isometric embeddings such that one has $\sup _{x \in X} d(f(x), g(x))<\infty$ then $\partial(f)=\partial(g)$.

4) If $f: X \rightarrow Y$ is a quasi-isometry then $\partial(f): \partial X \rightarrow \partial Y$ is a homeomorphism.

The next lemma is a consequence of the stability of quasi-geodesics (Lemma 1.26) in hyperbolic metric spaces.

Lemma 2.3. Let $X$ be a $\delta$-hyperbolic metric space and let $\gamma:[0, \infty) \rightarrow X$ be a $(K, \epsilon)$-quasi geodesic ray. Let $\left\{t_{n}\right\}$ be any sequence of non-negative real numbers tending to $\infty$; then $\gamma\left(t_{n}\right) \rightarrow \infty$ and the point of $\partial X$ represented by $\left\{\gamma\left(t_{n}\right)\right\}$ is independent of the sequence $\left\{t_{n}\right\}$. 
The point of $\partial X$ determined by a quasi-geodesic ray $\gamma$ will be denoted by $\gamma(\infty)$. The next lemma constructs quasigeodesic rays joining points in $X$ to points in $\partial X$ as well as bi-infinite quasigeodesics joining pairs of points in $\partial X$. While this is standard for proper $X$ Gd90, ready references for arbitrary (non-proper) $X$ are a bit difficult to come by, and we include a proof for completeness.

Lemma 2.4. For any $\delta \geq 0$ there is a constant $\left.K=K \overline{2.4}^{(} \delta\right)$ such that the following holds:

Suppose $X$ is a $\delta$-hyperbolic metric space.

(1) Given any point $\xi \in \partial X$ and $p \in X$ there is a $K$-quasi-geodesic ray $\gamma$ : $[0, \infty) \rightarrow X$ of $X$ with $\gamma(0)=p$ and $\gamma(\infty)=\xi$.

(2) Given two points $\xi_{1} \neq \xi_{2} \in \partial X$ there is a $K$-quasi-geodesic line $\alpha: \mathbb{R} \rightarrow X$ with $\alpha(-\infty)=\xi_{1}$ and $\alpha(\infty)=\xi_{2}$.

Terminology: Any quasi-geodesic ray as in (1) of the above lemma will be referred to as a quasi-geodesic ray joining the points $p$ and $\xi$. Similarly any quasi-geodesic as in (2) of the above lemma will be referred to as a quasi-geodesic line joining the points $\xi_{1}$ and $\xi_{2}$.

Proof of Lemma 2.4: (1). We shall inductively construct a suitable sequence of points $\left\{p_{n}\right\}$ such that $p_{n} \rightarrow \xi$ and finally show that the union $\cup\left[p_{n}, p_{n+1}\right]$, of the geodesic segments $\left[p_{n}, p_{n+1}\right]$, is a uniform quasi-geodesic. Suppose $x_{n} \rightarrow \xi, x_{n} \in X$, for all $n$. Fix $N \geq 1$ and let $p_{0}=p$. Since $x_{n} \rightarrow \infty$ we can find a positive integer $n_{1} \in \mathbb{N}$ such that $\left(x_{i} . x_{j}\right)_{p_{0}} \geq N$ for all $i, j \geq n_{1}$. Let $\left[p_{0}, x_{n_{1}}\right]$ be a geodesic joining $p_{0}$ and $x_{n_{1}}$. Choose $p_{1} \in\left[p_{0}, x_{n_{1}}\right]$ such that $d\left(p_{0}, p_{1}\right)=N$. Now suppose $p_{l}$ has been constructed. To construct $p_{l+1}$, let $n_{l+1} \geq \max \left\{n_{k}: 1 \leq k \leq l\right\}$ be an integer such that $\left(x_{i} . x_{j}\right)_{p_{l}} \geq(l+1) N$ for all $i, j \geq n_{l+1}$. Choose $p_{l+1} \in\left[p_{l}, x_{n_{l+1}}\right]$ such that $d\left(p_{l}, p_{l+1}\right)=(l+1) N$. Now, let $\alpha_{N}$ be the arc length parametrization of the concatenation of the geodesic segments $\left[p_{i}, p_{i+1}\right], i \in \mathbb{Z}^{+}$.

Claim: For $N>\max \left\{7 \delta+1, \frac{1}{3} L 1.28(\delta, 1,42 \delta)\right\}, \alpha_{N}$ is a $\lambda_{1.28}(\delta, 1,42 \delta)$-quasigeodesic.

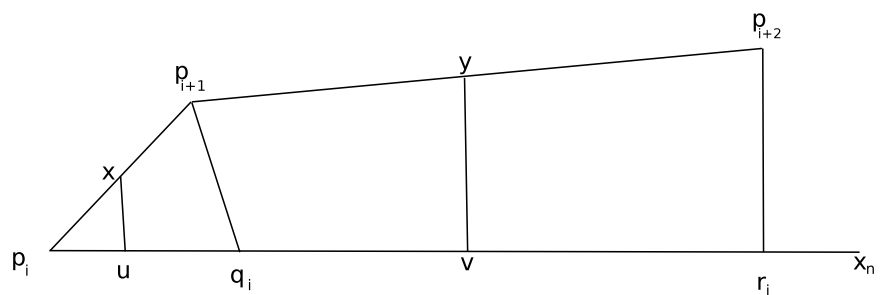

First we show that $\left[p_{i}, p_{i+1}\right] \cup\left[p_{i+1}, p_{i+2}\right]$ is a uniform quasi-geodesic for each $i$. Let $n>n_{i+2}$. Join $p_{i}$ with $x_{n}$. Since $\left(x_{n} . x_{n_{i+1}}\right)_{p_{i}} \geq(i+1) N$ and triangles in $X$ are $6 \delta$-thin by Lemma $1.25(2)$, we can find a point $q_{i} \in\left[p_{i}, x_{n}\right]$ such that $d\left(p_{i}, q_{i}\right)=(i+1) N$ and $d\left(p_{i+1}, q_{i}\right) \leq 6 \delta$. Similarly there is a point $q_{i+1} \in\left[p_{i+1}, x_{n}\right]$ such that $d\left(p_{i+1}, q_{i+1}\right)=(i+2) N$ and $d\left(p_{i+2}, q_{i+1}\right) \leq 6 \delta$.

Consider the triangle $\Delta p_{i} p_{i+1} x_{n}$. The point $q_{i+1} \in\left[p_{i+1}, x_{n}\right]$ is contained in a $\delta$-neighborhood of $\left[p_{i}, p_{i+1}\right] \cup\left[p_{i}, x_{n}\right]$. Hence there exists $r_{i} \in\left[p_{i}, x_{n}\right] \cup\left[p_{i}, p_{i+1}\right]$ such that $d\left(r_{i}, q_{i+1}\right) \leq \delta$. Since $d\left(q_{i+1}, p_{i+1}\right)=(i+2) N$, it follows from the triangle inequality that $d\left(r_{i}, q_{i}\right) \geq d\left(q_{i+1}, p_{i+1}\right)-d\left(r_{i}, q_{i+1}\right)-d\left(p_{i+1}, q_{i}\right) \geq(i+2) N-\delta-6 \delta$. Again, since $N>7 \delta+1$, it follows that $d\left(r_{i}, q_{i}\right)>(i+1) N+1$ and hence $r_{i} \notin$ $\left[p_{i}, q_{i}\right]\left(\subset\left[p_{i}, x_{n}\right]\right)$. 
Next, we note that $r_{i} \notin\left[p_{i}, p_{i+1}\right]$. Else suppose $r_{i} \in\left[p_{i}, p_{i+1}\right]$. Then $(i+1) N=$ $d\left(p_{i}, p_{i+1}\right) \geq d\left(r_{i}, p_{i+1}\right) \geq d\left(p_{i+1}, q_{i+1}\right)-d\left(r_{i}, q_{i+1}\right) \geq(i+2) N-\delta$. This is a contradiction since $N>7 \delta+1$. Thus $r_{i} \in\left[q_{i}, x_{n}\right] \subset\left[p_{i}, x_{n}\right]$. Also note that $d\left(p_{i+2}, r_{i}\right) \leq d\left(p_{i+2}, q_{i+1}\right)+d\left(q_{i+1}, r_{i}\right) \leq 7 \delta$.

We now show that $\left[p_{i}, p_{i+1}\right] \cup\left[p_{i+1}, p_{i+2}\right]$ is a $(1,42 \delta)$-quasi-geodesic of length at least $3 N$ for $N>7 \delta+1$. Suppose $x \in\left[p_{i}, p_{i+1}\right]$ and $y \in\left[p_{i+1}, p_{i+2}\right]$. It is enough to show that $\left|d\left(x, p_{i+1}\right)+d\left(p_{i+1}, y\right)-d(x, y)\right| \leq 42 \delta$.

For the $\delta$-slim triangle $\triangle p_{i} q_{i} p_{i+1}$, there exists $u \in\left[p_{i}, q_{i}\right]$ such that $d(x, u) \leq 7 \delta$. Similarly for $\triangle p_{i+1} q_{i} r_{i}$ and $\triangle p_{i+1} p_{i+2} r_{i}$, there exists $v \in\left[q_{i}, r_{i}\right]$ such that $d(y, v) \leq$ $8 \delta$ (the precise constant is obtained by a routine computation).

We have the following inequalities:

$\left|d\left(x, p_{i+1}\right)-d\left(u, q_{i}\right)\right| \leq d(x, u)+d\left(p_{i+1}, q_{i}\right) \leq 6 \delta+7 \delta=13 \delta$,

$\left|d\left(p_{i+1}, y\right)-d\left(q_{i}, v\right)\right| \leq d\left(p_{i+1}, q_{i}\right)+d(y, v) \leq 7 \delta+8 \delta=15 \delta$,

and $|d(u, v)-d(x, y)| \leq d(x, u)+d(y, v) \leq 6 \delta+8 \delta=14 \delta$.

Hence $\left|d\left(x, p_{i+1}\right)+d\left(p_{i+1}, y\right)-d(x, y)\right| \leq \mid\left\{d\left(x, p_{i+1}\right)-d\left(u, q_{i}\right)\right\}+\left\{d\left(p_{i+1}, y\right)-\right.$ $\left.d\left(q_{i}, v\right)\right\}+\{d(u, v)-d(x, y)\} \mid \leq 42 \delta$ and we are done.

The claim follows from Lemma 1.28,

Next we show that $\gamma(\infty)=\xi$. For this, by Lemma 2.3 we just need to check that $\left\{p_{n}\right\} \sim\left\{x_{n}\right\}$. Again, to show this, it is enough to check that $\left\{p_{k}\right\} \sim\left\{x_{n_{k-1}}\right\}$, i.e. $\lim _{k \rightarrow \infty}\left(p_{k} . x_{n_{k-1}}\right)_{p}=\infty$. By the above proof we know that $\left(\cup_{i=1}^{k-1}\left[p_{i-1}, p_{i}\right]\right) \bigcup\left[p_{k-1}, x_{n_{k-1}}\right]$ is a uniform quasi-geodesic. Thus, by stability of quasi-geodesics (Lemma 1.26), we can find a constant $D$ depending only on $\delta$ such that there is a point $u \in\left[p, x_{n_{k-1}}\right]$ with $d\left(p_{k-1}, u\right) \leq D$; similarly there is a point $v \in\left[p, p_{k}\right]$ such that $d\left(p_{k-1}, v\right) \leq D$. Therefore, we have $d(u, v) \leq 2 D$ and $\left(p_{k} \cdot x_{n_{k-1}}\right)_{p} \geq(u \cdot v)_{p} \geq d(p, u)-d(u, v) \geq$ $d\left(p, p_{k-1}\right)-d\left(u, p_{k-1}\right)-d(u, v) \geq d\left(p, p_{k-1}\right)-3 D$. As $\lim _{k \rightarrow \infty} d\left(p, p_{k}\right)=\infty$, we have $\lim _{k \rightarrow \infty}\left(p_{k} \cdot x_{n_{k-1}}\right)_{p}=\infty$. Therefore, the proof is complete by taking $K[2.4(\delta) \geq \lambda \overline{1.28}(\delta, 1,42 \delta)$.

(2) Let $\lambda:=\sqrt{1.28}(\delta, 1,42 \delta)$, and $D_{1}:=D \overline{1.26}(\delta, \lambda)$. Now, using the proof of (1), we can construct two $\lambda$-quasi-geodesic rays $\gamma_{1}, \gamma_{2}$, parametrized by arc length, joining a point $p \in X$ to $\xi_{1}$ and $\xi_{2}$ respectively. Clearly, $\sup \left\{(x . y)_{p}: x \in \gamma_{1}, y \in\right.$ $\left.\gamma_{2}\right\}<\infty$, else there exist $x_{n} \in \gamma_{1}, y_{n} \in \gamma_{2}, n \in \mathbb{N}$, such that $\left(x_{n} . y_{n}\right)_{p} \rightarrow \infty$. Since $x_{n} \rightarrow \gamma_{1}(\infty)=\xi_{1}$ and $y_{n} \rightarrow \gamma_{2}(\infty)=\xi_{2}$ by Lemma 2.3. this contradicts the fact that $\xi_{1} \neq \xi_{2}$.

Let $N_{1}=\sup \left\{(x . y)_{p}: x \in \gamma_{1}, y \in \gamma_{2}\right\}$. Let $x_{i} \in \gamma_{i}, i=1,2$, be such that $\left(x_{1} \cdot x_{2}\right)_{p} \geq N_{1}-1$. Let $u_{i} \in\left[p, x_{i}\right], i=1,2$ be internal points of $\Delta p x_{1} x_{2}$. By Lemma 1.26 we can find $p_{i} \in \widehat{x}_{i}$ such that $d\left(p_{i}, u_{i}\right) \leq D_{1}, i=1,2$. Now, let $\gamma_{i}^{\prime} \subset \gamma_{i}$ be the quasi-geodesic subray starting from $p_{i}$, for $i=1,2$. We intend to show that the arc length parametrization of the concatenation of $\gamma_{1}^{\prime}, \gamma_{2}^{\prime}$ and a geodesic segment $\left[p_{1}, p_{2}\right]$ joining $p_{1}, p_{2}$ is a uniform quasi-geodesic (see figure below). 


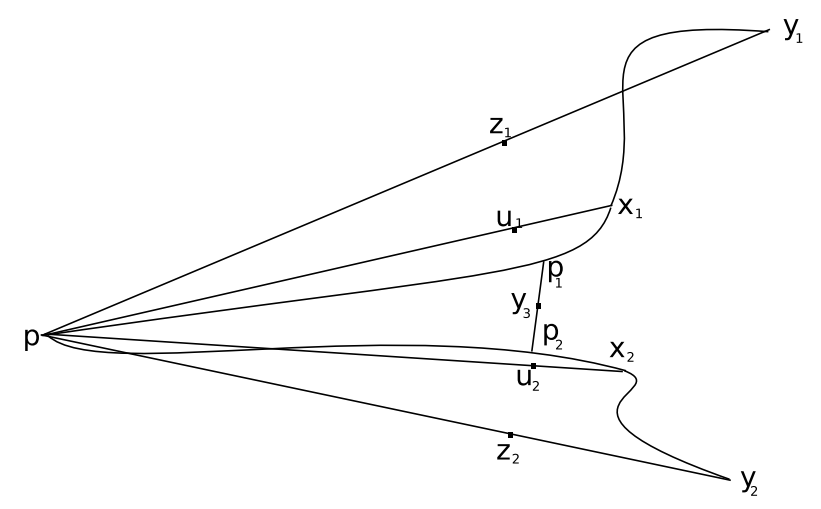

Suppose $y_{i} \in \gamma_{i}^{\prime}, i=1,2$ and $y_{3} \in\left[p_{1}, p_{2}\right]$. It suffices to find $K \geq 1$ and $\epsilon \geq 0$ independent of $y_{1}, y_{2}, y_{3}$ such that the following conditions are satisfied.

Condition (1) $l\left(\mathrm{p}_{1} y_{1}\right)+l\left(\widehat{p_{2} y_{2}}\right)+d\left(p_{1}, p_{2}\right) \leq K d\left(y_{1}, y_{2}\right)+\epsilon$,

Condition (2) $l\left(p_{1} \widehat{y}_{1}\right)+d\left(p_{1}, y_{3}\right) \leq K d\left(y_{1}, y_{3}\right)+\epsilon$, and

Condition $(3) l\left(p_{2} \widehat{y}_{2}\right)+d\left(y_{2}, y_{3}\right) \leq K d\left(y_{2}, y_{3}\right)+\epsilon$,

where $\widehat{p_{i} y_{i}}$ is the subsegement of $\gamma_{i}$ between $p_{i}$ and $y_{i}$ for $i=1,2$; also for a rectifiable curve segment $\alpha, l(\alpha)$ denotes the length of the curve $\alpha$. Since the proofs of Conditions (2) and (3) are similar we shall give proofs of Conditions (1) and (2) below.

First of all, we note that $d\left(u_{1}, u_{2}\right) \leq 4 \delta$ by Lemma 1.25 and hence $d\left(p_{1}, p_{2}\right) \leq$ $d\left(p_{1}, u_{1}\right)+d\left(p_{2}, u_{2}\right)+d\left(u_{1}, u_{2}\right) \leq 2 D_{1}+4 \delta=D_{2}$, say. By Lemma 1.26 we can find $z_{i} \in\left[p, y_{i}\right]$ such that $d\left(p_{i}, z_{i}\right) \leq D_{1}, i=1,2$.

We shall first show that the difference between $\left(y_{1} \cdot y_{2}\right)_{p}$ and $\left(p_{1} \cdot p_{2}\right)_{p}$ is small. Note that $\left(y_{1} \cdot y_{2}\right)_{p} \geq\left(z_{1} \cdot z_{2}\right)_{p} \geq\left(p_{1} \cdot p_{2}\right)_{p}-\left\{d\left(p_{1}, z_{1}\right)+d\left(p_{2}, z_{2}\right)\right\} \geq\left(p_{1} . p_{2}\right)_{p}-2 D_{1}$. Also, $\left|\left(x_{1} . x_{2}\right)_{p}-\left(u_{1} . u_{2}\right)_{p}\right|=\left|d\left(p, u_{1}\right)-\left(u_{1} . u_{2}\right)_{p}\right|=d\left(u_{1}, u_{2}\right) / 2 \leq 2 \delta$ and $\mid\left(p_{1} . p_{2}\right)_{p}-$ $\left(u_{1} . u_{2}\right)_{p} \mid \leq d\left(p_{1}, u_{1}\right)+d\left(p_{2}, u_{2}\right) \leq 2 D_{1}$. Thus $\left|\left(x_{1} \cdot x_{2}\right)_{p}-\left(p_{1} \cdot p_{2}\right)_{p}\right| \leq 2\left(D_{1}+\delta\right)$ and hence $\left(y_{1} \cdot y_{2}\right)_{p} \geq\left(p_{1} \cdot p_{2}\right)_{p}-2 D_{1} \geq\left(x_{1} \cdot x_{2}\right)_{p}-\left(4 D_{1}+2 \delta\right)$.

Since $\left(y_{1} \cdot y_{2}\right)_{p} \leq N_{1}$ and $\left(x_{1} \cdot x_{2}\right)_{p} \geq N_{1}-1$ we have

$$
\left|\left(y_{1} . y_{2}\right)_{p}-\left(p_{1} \cdot p_{2}\right)_{p}\right| \leq\left(1+2 \delta+4 D_{1}\right) .
$$

Next, suppose that $c_{i} \in\left[p, y_{i}\right], i=1,2$ and $c \in\left[y_{1}, y_{2}\right]$ are the internal points of $\Delta p y_{1} y_{2}$. We shall show that $d\left(p_{i}, c_{i}\right), i=1,2$ are small.

Suppose $q_{i} \in\left[p, p_{i}\right], i=1,2$ are internal points of $\triangle p p_{1} p_{2}$. Then $d\left(p_{i}, q_{i}\right) \leq$ $d\left(p_{1}, p_{2}\right) \leq D_{2}$. We can choose $r_{i} \in\left[p, y_{i}\right]$ such that $d\left(r_{i}, q_{i}\right) \leq 2 D_{1}$, by Lemma 1.26 applied to the subsegment of the quasi-geodesic $\gamma_{i}$ between $p, p_{i}$ and $p, y_{i}$. Hence $d\left(c_{i}, r_{i}\right)=\left|d\left(p, c_{i}\right)-d\left(p, r_{i}\right)\right| \leq\left|d\left(p, c_{i}\right)-d\left(p, q_{i}\right)\right|+\left|d\left(p, q_{i}\right)-d\left(p, r_{i}\right)\right| \leq$ $\left(1+2 \delta+4 D_{1}\right)+d\left(q_{i}, r_{i}\right)$. Hence $d\left(c_{i}, r_{i}\right) \leq\left(1+2 \delta+6 D_{1}\right)$. This gives $d\left(c_{i}, p_{i}\right) \leq$ $d\left(c_{i}, r_{i}\right)+d\left(r_{i}, q_{i}\right)+d\left(q_{i}, p_{i}\right) \leq\left(1+2 \delta+8 D_{1}+D_{2}\right)$. Since $d\left(c, c_{i}\right) \leq 4 \delta$ we have

$$
d\left(c, p_{i}\right) \leq d\left(c, c_{i}\right)+d\left(c_{i}, p_{i}\right) \leq\left(1+6 \delta+8 D_{1}+D_{2}\right) .
$$

Thus for any point $y_{3} \in\left[p_{1}, p_{2}\right]$ we have $d\left(c, y_{3}\right) \leq d\left(p_{1}, p_{2}\right)+d\left(p_{1}, c\right) \leq(1+6 \delta+$ $\left.8 D_{1}+2 D_{2}\right)=D_{3}$, say. 
Proof of Condition 1 : Now,

$$
\begin{aligned}
& \sum_{i=1}^{2} l\left({\widehat{p_{i}}}_{i}\right)+d\left(p_{1}, p_{2}\right) \\
& \leq \sum_{i=1}^{2}\left\{\lambda d\left(p_{i}, y_{i}\right)+\lambda\right\}+D_{2}, \text { since } \gamma_{i} \text { are } \lambda \text { - quasi-geodesics. } \\
& \leq \sum_{i=1}^{2} \lambda\left\{d\left(y_{i}, c\right)+d\left(c, p_{i}\right)\right\}+2 \lambda+D_{2} \\
& \leq \lambda d\left(y_{1}, y_{2}\right)+2 \lambda+D_{2}+2 \lambda D_{3} .
\end{aligned}
$$

Proof of Condition 2:

$$
\begin{aligned}
& l\left(\widehat{p}_{1} \hat{y}_{1}\right)+d\left(p_{1}, y_{3}\right) \\
& \leq\left\{\lambda d\left(p_{1}, y_{1}\right)+\lambda\right\}+d\left(p_{1}, y_{3}\right) \\
& \leq \lambda\left\{d\left(y_{1}, y_{3}\right)+d\left(y_{3}, p_{1}\right)\right\}+\lambda+d\left(p_{1}, y_{3}\right) \\
& \leq \lambda d\left(y_{1}, y_{3}\right)+\lambda+(\lambda+1) d\left(p_{1}, p_{2}\right) \\
& \leq \lambda d\left(y_{1}, y_{3}\right)+\left(\lambda+(\lambda+1) D_{2}\right) .
\end{aligned}
$$

As mentioned before, the proof of Condition 3 is exactly like the proof of Condition 2 .

Two quasi-geodesic rays $r_{i}:[0, \infty) \rightarrow X, i=1,2$, in a hyperbolic metric space $(X, d)$ are said to be asymptotic if there exists $C_{0}$ such that $d\left(r_{1}(t), r_{2}(t)\right) \leq C_{0}$ for all $t \in[0, \infty)$. Using stability of quasi-geodesics (Lemma 1.26) the proofs of the following lemma and corollary are standard (see Lemma 1.15, Chapter III.H, BH99).

Lemma 2.5. Asymptotic rays are uniformly close: For all $\delta \geq 0$ and $k \geq 1$ there is a constant $D \overline{2.5}=\overline{2.5}(\delta, k)$ such that the following holds:

Suppose $X$ is a $\delta$ hyperbolic metric space and $\gamma_{1}, \gamma_{2}:[0, \infty) \rightarrow X$ are two asymptotic $k$-quasi-geodesic rays. Then there exists $T \geq 0$ such that $\gamma_{1}(t) \in N_{D_{2.5}}\left(\operatorname{Im}\left(\gamma_{2}\right)\right)$ and $\gamma_{2}(t) \in N_{[2.5]}\left(\operatorname{Im}\left(\gamma_{1}\right)\right)$, for all $t \geq T$.

Corollary 2.6. For all $\delta \geq 0$ and $K \geq 1$ there is a constant $D \overline{2.6}=D_{2.6}(\delta, K)$ such that the following holds:

Suppose $X$ is a $\delta$-hyperbolic metric space and let $\gamma_{1}, \gamma_{2}$ be two $K$-quasi-geodesic lines in $X$ joining the same pair of points $\xi_{1}, \xi_{2} \in \partial X$. Then the Hausdorff distance between $\gamma_{1}$ and $\gamma_{2}$ is at most $D \overline{2.6}$.

Lemma 1.26 and Lemma 2.5 combined with the proof of Lemma 1.29(2), immediately imply the following result.

Lemma 2.7. For all $\delta \geq 0, D^{\prime} \geq 0$ and $k \geq 1$ there are constants $D=D \overline{2.7}(\delta, k)$ and $L=L \overline{2.7}\left(\delta, k, D^{\prime}\right)$ such that we have the following:

Suppose $X$ is a $\delta$-hyperbolic metric space. Then

(1) Let $\Delta \xi_{1} \xi_{2} \xi_{3}$ be a $k$-quasi-geodesic ideal triangle in $X$, i.e. a union of three $k$-quasi-geodesic lines in $X$ joining the pairs of points $\left(\xi_{i}, \xi_{j}\right), i \neq j ; 1 \leq$ $i, j \leq 3$. Let us denote the quasi-geodesic lines joining $\xi_{i}, \xi_{j}$ by $\left[\xi_{i}, \xi_{j}\right]$. Then there is a point $x \in X$ such that $x \in N_{D}\left(\left[\xi_{i}, \xi_{j}\right]\right)$ for all $i \neq j$.

(2) If $x, x^{\prime} \in X$ are two points each of which is contained within a $D^{\prime}-$ neighborhood of each of the sides of an ideal k-quasi-geodesic triangle $\Delta \xi_{1} \xi_{2} \xi_{3}$ then $d\left(x, x^{\prime}\right) \leq L$.

If a point $x \in X$ is contained in the $D^{\prime}$-neighborhood of each of the sides of an ideal quasi-geodesic triangle $\Delta \xi_{1} \xi_{2} \xi_{3}$, then $x$ will be called a $D^{\prime}$-barycenter of

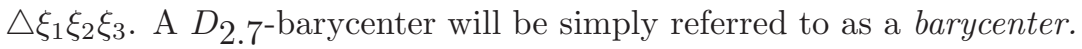

Now, Lemma 2.7 along with the proof of Lemma 1.29)(2) gives the following. 
Lemma 2.8. Given $\delta \geq 0, D^{\prime} \geq 0, K_{1} \geq 1$ and $K_{2} \geq 0$ there exists $D=$ $D\left(\delta, K_{1}, K_{2}, D^{\prime}\right)$ such the following holds:

Suppose $f: X \rightarrow Y$ is a $K_{1}$-quasi isometric embedding of $\delta$-hyperbolic metric spaces. Let $\triangle \xi_{1} \xi_{2} \xi_{3} \subset X$ and $\triangle \partial(f)\left(\xi_{1}\right) \partial(f)\left(\xi_{2}\right) \partial(f)\left(\xi_{3}\right) \subset Y$ be $K_{2}$-quasi-geodesic ideal triangles. If $x \in X$ is a $D^{\prime}$-barycenter of $\Delta \xi_{1} \xi_{2} \xi_{3}$, then $f(x) \in Y$ is a $D$ barycenter of $\Delta \partial(f)\left(\xi_{1}\right) \partial(f)\left(\xi_{2}\right) \partial(f)\left(\xi_{3}\right)$.

\section{The barycenter map}

Suppose $X$ is a $\delta$-hyperbolic metric space such that $\partial X$ has more than two points. Let us denote the set of all distinct triples of points in $\partial X$ by $\partial^{3} X$. Now, given $\xi=\left(\xi_{1}, \xi_{2}, \xi_{3}\right) \in \partial^{3} X$ we can, by Lemma 2.4 construct a $K_{2.4}(\delta)$-quasi-geodesic ideal triangle, say $\Delta_{1}$, with vertices $\xi_{i}, i=1,2,3$. Then, by Lemma 2.7(2) there is a coarsely well defined barycenter of $\Delta_{1}$. Suppose $b_{\xi}$ is a barycenter of $\Delta_{1}$. Henceforth, we shall refer to it simply as a barycenter of the triple $\left(\xi_{1}, \xi_{2}, \xi_{3}\right)$. For a different set of choices of the $K_{2.4}(\delta)$-quasi-geodesic lines joining the pairs $\left(\xi_{i}, \xi_{j}\right)$, suppose we obtain a new ideal triangle $\Delta_{2}$, and suppose $b_{\xi}^{\prime}$ is a barycenter of $\left(\xi_{1}, \xi_{2}, \xi_{3}\right)$ defined with respect to $\Delta_{2}$. Then by the stability of quasi-geodesic lines (Corollary 2.6), $b_{\xi}^{\prime}$ is a $D_{1}:=\left(D_{2.7}(\delta)+D_{2.6}\left(\delta, K{ }_{2.4}(\delta)\right)\right.$-barycenter of the triangle $\Delta_{1}$. Hence, by Lemma $2.7(2), d\left(b_{\xi}, b_{\xi}^{\prime}\right) \leq L_{2.7}\left(\delta, K_{2.4}(\delta), D_{1}\right)$ and we have:

Lemma 2.9. For every $\delta \geq 0$ there is a constant $D \overline{2.9}=D \overline{2.9}(\delta)$ such that we have the following:

Suppose $X$ is a $\delta$-hyperbolic metric space and $\xi=\left(\xi_{1}, \xi_{2}, \xi_{3}\right) \in \partial^{3} X$. If $b_{\xi}$ and $b_{\xi}^{\prime}$ are two barycenters of $\xi$, then $d\left(b_{\xi}, b_{\xi}^{\prime}\right) \leq D 2 \overline{2.9}$.

We shall say that a map $f: U \rightarrow\left(V, d_{V}\right)$ satisfying properties $\mathcal{P}_{1}, \cdots, \mathcal{P}_{k}$ is coarsely unique if there exists $C>0$ such that for any other map $g: U \rightarrow\left(V, d_{V}\right)$ satisfying properties $\mathcal{P}_{1}, \cdots, \mathcal{P}_{k}$, and any $u \in U, d_{V}(f(u), g(u)) \leq C$.

Thus, from Lemma 2.9 we have a coarsely unique map $\phi: \partial^{3} X \rightarrow X, \xi \mapsto b_{\xi}$ mapping a triple of points to a barycenter. Any such map will be referred to as the barycenter map. Now we are ready to state the main proposition of this subsection.

Proposition 2.10. Existence of qi sections for metric graph bundles: For all $\delta^{\prime}, N \geq 0$ and proper $f: \mathbb{N} \rightarrow \mathbb{N}$ there exists $K_{0}=K_{0}\left(f, \delta^{\prime}, N\right)$ such that the following holds.

Suppose $p: X \rightarrow B$ is an $(f, K)$-metric graph bundle with the following properties:

(1) Each of the fibers $F_{b}, b \in \mathcal{V}(B)$ is a $\delta^{\prime}$-hyperbolic metric space with respect to the path metric $d_{b}$ induced from $X$.

(2) The barycenter maps $\phi_{b}: \partial^{3} F_{b} \rightarrow F_{b}$ are uniformly coarsely surjective, i.e. $F_{b}$ is contained in the $N$-neighborhood of the image of $\phi_{b}$ for all $b \in \mathcal{V}(B)$.

Then there is a $K_{0}$-qi section through each point of $\mathcal{V}(X)$.

Note that the constant $K$ in Proposition 2.10 above is given by $K=f(4)$ by Proposition 1.7 and hence we may write $K_{0}=K_{0}\left(f, K, \delta^{\prime}, N\right)$ by making the implicit dependence on $K$ explicit. We also assume without loss of generality that for all $b \in \mathcal{V}(B)$, the image of $\phi_{b}$ is contained in $\mathcal{V}\left(F_{b}\right)$.

Proof. Let us fix a set $\left\{\phi_{b}\right\}_{b \in \mathcal{V}(B)}$ of barycenter maps and let $v \in \mathcal{V}(B), x \in \mathcal{V}\left(F_{v}\right)$. First, suppose that $x$ is contained in the image of the barycenter map $\phi_{v}$. We will construct a qi section through $x$. Choose a point $\xi_{v}=\left(\xi_{1}, \xi_{2}, \xi_{3}\right) \in \partial^{3} F_{v}$ such that $\phi_{v}\left(\xi_{v}\right)=x$. Denote $\xi_{v}=\xi$ and so $\phi_{v}(\xi)=x$. 
Let $w, z \in \mathcal{V}(B), w \neq z$. Choose a geodesic $\gamma$ joining $w, z$ and let $w=$ $w_{0}, w_{1}, \ldots, w_{n-1}, w_{n}=z$ be the consecutive vertices on $\gamma$. By condition $(2)(i i)$ of the definition of metric graph bundles (Proposition[1.7), for all $i, 0 \leq i \leq n-1$, there is a $K$-quasi-isometry $f_{w_{i} w_{i+1}}: F_{w_{i}} \rightarrow F_{w_{i+1}}$ which sends any vertex $y_{i} \in \mathcal{V}\left(F_{w_{i}}\right)$ to a vertex $y_{i+1} \in \mathcal{V}\left(F_{w_{i+1}}\right)$ where $y_{i}$ and $y_{i+1}$ are connected by an edge. By composition of $n$ such maps we get a map $f_{w z}: F_{w} \rightarrow F_{z}$, which sends each point $y \in F_{w}$ to a point $y^{\prime} \in \mathcal{V}\left(F_{z}\right)$ such that $d\left(y, y^{\prime}\right) \leq d_{B}(w, z)+1=n+1$. Let $f_{w w}: F_{w} \rightarrow F_{w}$ denote the identity map on $F_{w}$, for all $w \in \mathcal{V}(B)$. Now we make the following observations:

1. Since the inclusion maps $F_{w} \hookrightarrow X$ are uniformly metrically proper, by the definition of metric graph bundles, the map $f_{w z}$ is coarsely uniquely determined. In fact, if $d(w, z)=n, n \in \mathbb{N}$, we have for any other map $f_{w z}^{\prime}$ defined in the same way, $d\left(f_{w z}(y), f_{w z}^{\prime}(y)\right) \leq 2(n+1)$, so that $d_{z}\left(f_{w z}(y), f_{w z}^{\prime}(y)\right) \leq f(2 n+2)$, for all $y \in F_{w}$.

2. Since each $f_{w z}$ is obtained as a composition of $K$-quasi-isometries it is a quasi-isometry. Now, since the spaces $F_{w}, w \in \mathcal{V}(B)$, are $\delta^{\prime}$-hyperbolic and since the map $f_{w z}$ is coarsely uniquely determined, we have a well defined map $\partial\left(f_{w z}\right)$ : $\partial F_{w} \rightarrow \partial F_{z}$, by Lemma 2.2 and hence an induced map $\partial^{3} f_{w z}: \partial^{3} F_{w} \rightarrow \partial^{3} F_{z}$, $\forall w, z \in \mathcal{V}(B)$.

Consider the map $s=s_{\xi, x}: \mathcal{V}(B) \rightarrow X$ given by $s(v)=x=\phi_{v}(\xi)$, and $s(w)=\phi_{w}\left(\left(\partial^{3} f_{v w}(\xi)\right)\right)$, for all $w \in \mathcal{V}(B), w \neq v$. We show below that $s$ (or $s(\mathcal{V}(B)))$ is the required qi section through $x$.

3. Writing $\xi_{w}=\partial^{3} f_{v w}(\xi)$ for all $w \neq v$, note that for any $w, z \in \mathcal{V}(B)$, $\partial^{3} f_{w z}\left(\xi_{w}\right)=\xi_{z}$. This follows from the fact that by the definitions of the maps $f_{v z}$, $f_{w z}, f_{v w}$ we have, for all $y \in F_{v}, d\left(f_{v z}(y), f_{w z} \circ f_{v w}(y)\right) \leq d_{B}(v, z)+d_{B}(w, z)+$ $d_{B}(v, w)+3$, and thus $d_{z}\left(f_{v z}(x), f_{w z} \circ f_{v w}(x)\right) \leq f\left(d_{B}(v, z)+d_{B}(w, z)+d_{B}(v, w)+3\right)$. The claim follows from Lemma $2.2(3)$.

4. Lastly, we show that there exists $C \geq 1$ such that for any pair $w, z$ of adjacent vertices of $B, d(s(w), s(z)) \leq C$. By Condition 2 (ii) (Proposition 1.7) $f_{w z}$ is a $K$-quasi-isometry. Let $\xi_{w}=\left(\beta_{1}, \beta_{2}, \beta_{3}\right)$ and $\partial^{3} f_{w z}\left(\xi_{w}\right)=\xi_{z}=\left(\eta_{1}, \eta_{2}, \eta_{3}\right)$. Choose $K\left[2.4\left(\delta^{\prime}\right)\right.$-quasigeodesic ideal triangles $\Delta_{w}$ and $\Delta_{z}$ respectively in $F_{w}$ and $F_{z}$, with vertices $\xi_{i}$ 's and $\eta_{i}$ 's; by definition of the map $s, s(w)$ and $s(z)$ are $D 2.7\left(\delta^{\prime}\right)$-barycenters of these triangles. Now, the map $f_{w z}$ takes the ideal triangle $\Delta_{w}$ to an ideal $K_{1}$-quasigeodesic triangle with vertices $\eta_{i}$ 's, where $K_{1}=$ $K\left[2.4\left(\delta^{\prime}\right) \cdot K+K\right.$, and $f_{w z}(s(w))$ is a $D_{1}:=\left\{D \overline{2.7}\left(\delta^{\prime}\right) \cdot K+K\right\}$-barycenter of the new triangle. Thus, by Lemma 2.6 $f_{w z}(s(w))$ is a $D_{2}$-barycenter of the triangle $\Delta_{z}$, where $D_{2}=D \overline{2.6}\left(\delta^{\prime}, K_{1}\right)+D_{1}$. Hence, by Lemma 2.7. we have $d\left(s(z), f_{w z}(s(w))\right) \leq$ $L 2.7\left(\delta^{\prime}, K\left[2.4\left(\delta^{\prime}\right), D_{2}\right)\right.$. Since $d\left(s(w), f_{w z}(s(w))\right)=1$ we have $d(s(w), s(z)) \leq C:=$ $1+L 2.7\left(\delta^{\prime}, K / 2.4\left(\delta^{\prime}\right), D_{2}\right)$.

For any $w, z \in \mathcal{V}(B), d(w, z) \leq d(s(w), s(z))$ by the definition of a metric graph bundle. Also from Step (4) above, we have $d(s(w), s(z)) \leq C . d(w, z)$. Hence $s$ is a $C$-qi section.

If $x \in \mathcal{V}\left(F_{v}\right)$ is not in the image of $\phi_{v}$, we can choose $x_{1} \in \mathcal{V}\left(F_{v}\right)$ such that $d\left(x, x_{1}\right) \leq N$ and $x_{1} \in \operatorname{Im}\left(\phi_{v}\right)$. Now construct as above a $C$-qi section $s=s_{\xi, x_{1}}$, and define a new section $s^{\prime}$ by setting $s^{\prime}(b)=s(b)$ for all $b \in \mathcal{V}(B), b \neq v$ and $s^{\prime}(v)=x$. This is an $(N+C)$-qi section passing through $x$. Thus we can take $K_{0}=N+C$ to finish the proof of the proposition. 
Applying this proposition to Example 1.8, we have a different proof of the following result of Mosher Mos96.

Theorem 2.11. (Mosher Mos96) Let us consider the short exact sequence of finitely generated groups

$$
1 \rightarrow A \rightarrow G \rightarrow Q \rightarrow 1 .
$$

such that $A$ is non-elementary word hyperbolic. Then there exists a q(uasi)-i(sometric) section $\sigma: Q \rightarrow G$. Hence, if $G$ is hyperbolic, then so is $Q$.

Let $p: X^{\prime} \rightarrow B^{\prime}$ be an $(f, c, K)$-metric bundle and let $\pi: X \rightarrow B$ be an approximating metric graph bundle as in Lemma 1.21. As in Lemma 1.21 we suppose that the maps $\psi_{X}, \psi_{B}$ are $K_{1}-$ quasi-isometries. Let $\psi_{X^{\prime}}\left(\right.$ resp. $\left.\psi_{B^{\prime}}\right)$ be a quasi-isometric coarse inverse of the map $\psi_{X}$ (resp. $\psi_{B}$ ) constructed as in the proof of Lemma 1.1 (2). We assume that these maps are inverses of $\psi_{X}, \psi_{B}$ when restricted to the vertex sets $\mathcal{V}(X)$ and $\mathcal{V}(B)$ respectively. Moreover, we assume that $\psi_{X^{\prime}}, \psi_{B^{\prime}}$ are $K_{1}$-quasi-isometries. Also we assume that the restrictions of $\psi_{X}$ and $\psi_{X^{\prime}}$ to horizontal spaces ( $c f$. Lemma 1.19) are $K_{1}$-quasi-isometries.

Proposition 2.12. Existence of qi section for metric bundles: Let $p: X^{\prime} \rightarrow$ $B^{\prime}, \pi: X \rightarrow B$ and $\psi_{B}, \psi_{X}, \psi_{X^{\prime}}, \psi_{B^{\prime}}, K_{1}$ be as above. Let $V \subset B^{\prime}$ be the collection of points of $B^{\prime}$ that form the vertex set of $B$. Suppose we have a $k$-qi section $s: V \rightarrow X$. Then we have a $k^{\prime}=K\left[2.12\left(f, c, K, K_{1}, k\right)\right.$-qi section $s^{\prime}: B^{\prime} \rightarrow X^{\prime}$ such that $\psi_{X} \circ s=s^{\prime} \circ \psi_{B}$.

Hence any metric bundle satisfying the properties

1) horizontal spaces are uniformly hyperbolic, and

2) the barycenter maps of these spaces are uniformly coarsely surjective admits a uniform qi section through each point.

Proof. The proof of the first part of the proposition is clear once we describe what the map $s^{\prime}$ is. For $u \in V$ define $s^{\prime}(u)=\psi_{B^{\prime}} \circ s(u)$. Suppose $u \in B \backslash V$. Let $v \in V$, so that $d(u, v) \leq 1$. Choose $x \in F_{v}$ such that $x$ can be joined to $s^{\prime}(u)$ by a curve in $X^{\prime}$ of length at most $c$ and define $s^{\prime}(v)=x$.

Next note that if the fibers of a metric bundle are (uniformly) hyperbolic, then so are the vertex spaces of an approximating metric graph bundle. This is because the fibers of an approximating metric graph bundle are uniformly quasi-isometric to the fibers of the metric bundle. Next (for the same reason) observe that if the barycenter maps of the metric bundle are uniformly coarsely surjective, then so are the barycenter maps of an approximating metric graph bundle. The last part of the proposition now follows from Proposition 2.10 and the first part of the proposition.

2.2. Ladders. We use the term ladder below due to a similar ladder construction in Mit98b. The term girth is taken from [BF92.

Definition 2.13. Suppose $X$ is a metric bundle (resp. a metric graph bundle) over $B$. Suppose $X_{1}$ and $X_{2}$ are two $c_{1}$-qi sections of the metric bundle $X$. For each $b \in B$ (resp. $b \in \mathcal{V}(B)$ ), join the points $X_{1} \cap F_{b}, X_{2} \cap F_{b}$ by a geodesic in $F_{b}$. We denote the union of these geodesics by $C\left(X_{1}, X_{2}\right)$, and call it a ladder formed by the sections $X_{1}$ and $X_{2}$. 
Remark 2.14. If (as in the case of interest) the horizontal spaces are $\delta^{\prime}$-hyperbolic, for some $\delta^{\prime} \geq 0$, the Hausdorff distance between any pair of ladders determined by two given sections $X_{1}, X_{2}$ is uniformly bounded. In such a situation, $C\left(X_{1}, X_{2}\right)$ will refer to any one of them, and abusing notation we refer to $C\left(X_{1}, X_{2}\right)$ as the ladder determined by $X_{1}, X_{2}$.

For four qi sections $X_{i}, i=1,2,3,4$ we write $C\left(X_{3}, X_{4}\right) \subset C\left(X_{1}, X_{2}\right)$ to mean $C\left(X_{3}, X_{4}\right) \cap F_{b} \subset C\left(X_{1}, X_{2}\right) \cap F_{b}$ for all $b \in B($ or $\mathcal{V}(B))$.

Definition 2.15. Suppose $X_{1}$ and $X_{2}$ are two $c_{1}$-qi sections of a metric bundle (resp. metric graph bundle) $X$ over $B$. We define $d_{h}\left(X_{1}, X_{2}\right)=\inf \left\{d_{b}\left(F_{b} \cap X_{1}, F_{b} \cap\right.\right.$ $\left.\left.X_{2}\right): b \in B\right\}$ (resp. $\left.\inf \left\{d_{b}\left(F_{b} \cap X_{1}, F_{b} \cap X_{2}\right): b \in \mathcal{V}(B)\right\}\right)$ and call it the girth of any ladder $C\left(X_{1}, X_{2}\right)$, determined by $X_{1}, X_{2}$.

Definition 2.16. Neck of Ladders: Suppose $X$ is a metric bundle (resp. metric graph bundle) over $B$ and let $X_{1}, X_{2}$ be two qi sections. Let $C\left(X_{1}, X_{2}\right)$ be a ladder determined by $X_{1}, X_{2}$ and let $A \geq 0$. We define $U_{A}\left(X_{1}, X_{2}\right)$ to be the set $\{b \in B$ : $\left.d_{b}\left(X_{1} \cap F_{b}, X_{2} \cap F_{b}\right) \leq A\right\}$ (resp. $\left.\left\{b \in \mathcal{V}(B): d_{b}\left(X_{1} \cap F_{b}, X_{2} \cap F_{b}\right) \leq A\right\}\right)$ and call it the A-neck of the ladder $C\left(X_{1}, X_{2}\right)$.

A first aim of this subsection is to show that under suitable restrictions on a metric bundle or a metric graph bundle necks of ladders are quasi-convex subsets of the base space. The next lemma leads to one of the main tools (Lemma 2.22) for proving the combination theorem 4.3. This lemma originally appears in Ham05] in the context of metric fibrations. The proof that we give here is almost the same as that of [Ham05, nevertheless we include it for the sake of completeness. For convenience of exposition we suppress the dependence of the constants (defined in the following lemma) on the parameters $f, c, K$.

Lemma 2.17. Let $X$ be an $(f, c, K)$-metric bundle over $B$ satisfying $\left(M_{k}, \lambda_{k}, n_{k}\right)$ flaring for all $k \geq 1$ (cf. Definition 1.12), and let $\mu_{k}$ be the bounded flaring function (cf. Corollary 1.16). Then for all $c_{1} \geq 1$ and $R>1$ there are constants $D \overline{2.17}=D \overline{2.17}\left(c_{1}, R\right)$ and $K_{[2.17}=K_{[2.17}\left(c_{1}\right)$ such that the following holds:

Suppose $X_{1}, X_{2}$ are two $c_{1}$-qi sections of $B$ in $X$ and let $A \geq \max \left\{M_{c_{1}}, d_{h}\left(X_{1}, X_{2}\right)\right\}$.

(1) Let $\gamma:\left[t_{0}, t_{1}\right] \rightarrow B$ be a geodesic such that

a) $d_{\gamma\left(t_{0}\right)}\left(X_{1} \cap F_{\gamma\left(t_{0}\right)}, X_{2} \cap F_{\gamma\left(t_{0}\right)}\right)=A R$.

b) $\gamma\left(t_{1}\right) \in U_{A}:=U_{A}\left(X_{1}, X_{2}\right)$ but for all $t \in\left[t_{0}, t_{1}\right), \gamma(t) \notin U_{A}$.

Then the length of $\gamma$ is at most $D \overline{2.17}\left(c_{1}, R\right)$.

(2) $U_{A}$ is $K_{2.17}$ quasi-convex in $B$.

(3) If $d_{h}\left(X_{1}, X_{2}\right) \geq M_{c_{1}}$ then the diameter of the set $U_{A}$ is at most $D_{2.17}^{\prime}=$ $D_{2.17}^{\prime}\left(c_{1}, A\right)$.

For convenience of exposition we will write $\lambda$ for $\lambda_{c_{1}}, n$ for $n_{c_{1}}$ and $\mu$ for $\mu_{c_{1}}$ in the proof below. Also $l(\alpha)$ will denote the length of a curve $\alpha$.

Proof. Since $A \geq d_{h}\left(X_{1}, X_{2}\right), U_{A} \neq \emptyset$.

(1) Let $\phi:\left[t_{0}, t_{1}\right] \rightarrow \mathbb{R}$ be the function $t \mapsto d_{\gamma(t)}\left(X_{1} \cap F_{\gamma(t)}, X_{2} \cap F_{\gamma(t)}\right)$ and $t_{1}-t_{0}=n . L+\epsilon$ where $L \in \mathbb{Z}^{+}$and $0 \leq \epsilon<n$. Suppose $L \geq 3$. Consider the sequence $\phi\left(t_{0}+n i\right), i=1, \cdots, L$. Since $\phi\left(t_{0}+n . i\right) \geq M_{c_{1}}$, for all $i \in[1, L-1]$,

$$
\lambda \phi\left(t_{0}+n i\right) \leq \max \left\{\phi\left(t_{0}+n(i-1)\right), \phi\left(t_{0}+n(i+1)\right)\right\}
$$

by the flaring condition. 
Hence if $\phi\left(t_{0}+n\right)>\phi\left(t_{0}\right)$ then $\phi\left(t_{0}+n(i+1)\right) \geq \lambda \phi\left(t_{0}+n i\right)$ for all $i \in[1, L-1]$. Then, $\left.\phi\left(t_{0}+n L\right)\right) \geq \lambda^{L-1} \phi\left(t_{0}\right)$. Using bounded flaring (Corollary 1.16) we have, $\phi\left(t_{0}+n L\right) \leq \mu(n) \max \left\{\phi\left(t_{1}\right), 1\right\}$. Putting all these together and using the fact that $\phi\left(t_{1}\right) \leq A$ and $\phi\left(t_{0}\right)>A$, we have $L-1<\log (\mu(n)) / \log \lambda$.

Hence, $L \geq 3$ and $\phi\left(t_{0}+n\right)>\phi\left(t_{0}\right)$ implies

$$
l(\gamma)<n(L+1) \leq 2 n+n \cdot \log \mu(n) / \log \lambda .
$$

Suppose $\phi\left(t_{0}\right) \geq \phi\left(t_{0}+n\right)$ and let $k \leq L$ be the largest integer such that $\phi\left(t_{0}\right) \geq$ $\phi\left(t_{0}+n\right) \geq \cdots \geq \phi\left(t_{0}+k . n\right)$. If $k \geq 2$, applying the flaring condition we get $\phi\left(t_{0}+(i-1) n\right) \geq \bar{\lambda} \phi\left(t_{0}+i n\right)$ for all $i \in[\overline{1}, k-1]$. Then $\phi\left(t_{0}\right) \geq \lambda^{k-1} \phi\left(t_{0}+(k-1) n\right)>$ $\lambda^{k-1} A$. Therefore $k<1+\left\{\log \phi\left(t_{0}\right)-\log A\right\} / \log \lambda=1+\log R / \log \lambda$. Also, by the first part of the proof, $l\left(\left.\gamma\right|_{\left[t_{0}+k . n, t_{1}\right]}\right)<\max \{3 n, 2 n+n \log \mu(n) / \log \lambda\}$. Hence,

$$
l(\gamma) \leq n+n\{\log R / \log \lambda+\max \{3 n, 2 n+n \log (\mu(n)) / \log \lambda\}\} .
$$

Taking $D \overline{2.17}=D 2.17\left(c_{1}, R\right)$ as the right hand side of the above inequality, part (1) of the lemma is proved.

(2) Suppose $\gamma:\left[t_{0}, t_{1}\right] \rightarrow B$ is a geodesic joining two points of $U_{A}$, such that for all $t \in\left(t_{0}, t_{1}\right), \gamma(t) \notin U_{A}$. Without loss of generality, we may assume that $t_{1}-t_{0}>n$. Let $t_{2}=t_{0}+n$. Then by bounded flaring, we have $\phi\left(t_{2}\right) \leq \mu(n) \phi\left(t_{0}\right) \leq \mu(n)$. A. Again by the first part of the lemma $l\left(\left.\gamma\right|_{\left[t_{2}, t_{1}\right]}\right) \leq D \overline{2.17}\left(c_{1}, \phi\left(t_{2}\right) / A\right)$. Since, the function $D \overline{2.17}$ is increasing in the second variable, given that the first variable is fixed, we have $l(\gamma) \leq n+D \overline{2.17}\left(c_{1}, \mu(n)\right)$. Hence, taking $K_{2.17}\left(c_{1}\right)=n+$ $D \overline{2.17}\left(c_{1}, \mu(n)\right)$ we are through.

(3) Suppose $b_{1}, b_{2} \in U_{A}, d_{B}\left(b_{1}, b_{2}\right) / 2=L . n+\epsilon, 0 \geq \epsilon<n$. Let $\gamma:[-(L . n+$ $\epsilon),(L . n+\epsilon)] \rightarrow B$ be a geodesic joining $b_{1}, b_{2}$, so that $\gamma(0)$ is the midpoint of the geodesic $\gamma$. The bounded flaring condition gives $d_{\gamma(t)}\left(F_{\gamma(t)} \cap X_{1}, F_{\gamma(t)} \cap X_{2}\right) \leq$ A. $\mu(n)$ for $t=-L . n, L . n$.

As in the proof of the first part of the lemma, $d_{\gamma(t)}\left(F_{\gamma(t)} \cap X_{1}, F_{\gamma(t)} \cap X_{2}\right) \geq$ $\lambda^{L} . d_{\gamma(0)}\left(F_{\gamma(0)} \cap X_{1}, F_{\gamma(0)} \cap X_{2}\right)$ either for $t=L . n$ or for $t=-L . n$. Since $d_{\gamma(0)}\left(F_{\gamma(0)} \cap\right.$ $\left.X_{1}, F_{\gamma(0)} \cap X_{2}\right) \geq M_{c_{1}}$, it follows that $\lambda^{L} \cdot M_{c_{1}} \leq A \cdot \mu(n)$. Hence, $L \leq \log \left(A \cdot \mu(n) / M_{c_{1}}\right) / \log \lambda$.

This lemma has the following analog for metric graph bundles. We omit the proof since it is an exact replica of the proof of the previous lemma (see also Remark 2.19 below). We just need to point out that in the proof of the first part of the lemma the function $\phi$ should have $\left[t_{0}, t_{1}\right] \cap \mathbb{Z}$ as domain and for the latter parts it is useful to recall that in a graph, points on a geodesic refer to the vertices on the geodesic. Also, as in Lemma 2.17 above, we suppress the dependence of the constants on the parameters $f, K$.

Lemma 2.18. Let $X$ be an $(f, K)$-metric graph bundle over $B$ satisfying $\left(M_{k}, \lambda_{k}, n_{k}\right)$ flaring for all $k \geq 1$ (cf. Definition 1.12), and let $\mu_{k}$ be the bounded flaring function (cf. Corollary 1.14). Then for all $c_{1} \geq 1$ and $R>1$ there are constants $D \overline{2.18}=D_{2.18}\left(c_{1}, R\right)$ and $K_{2.18}=K_{2.18}\left(c_{1}\right)$ such that the following holds:

Suppose $X_{1}, X_{2}$ are two $c_{1}$-qi sections of $B$ in $X$ and let $A \geq \max \left\{M_{c_{1}}, d_{h}\left(X_{1}, X_{2}\right)\right\}$.

(1) Let $\gamma:\left[t_{0}, t_{1}\right] \rightarrow B$ be a geodesic, $t_{0}, t_{1} \in \mathbb{Z}$, such that

a) $d_{\gamma\left(t_{0}\right)}\left(X_{1} \cap F_{\gamma\left(t_{0}\right)}, X_{2} \cap F_{\gamma\left(t_{0}\right)}\right)=A R$.

b) $\gamma\left(t_{1}\right) \in U_{A}:=U_{A}\left(X_{1}, X_{2}\right)$ but for all $t \in\left[t_{0}, t_{1}\right) \cap \mathbb{Z}, \gamma(t) \notin U_{A}$.

Then the length of $\gamma$ is at most $D \overline{2.18}\left(c_{1}, R\right)$.

(2) $U_{A}$ is $K$ 2.18 quasi-convex in $B$. 
(3) If $d_{h}\left(X_{1}, X_{2}\right) \geq M_{c_{1}}$ then the diameter of the set $U_{A}$ is at most $D_{2.18}^{\prime}=$ $D_{2.18}^{\prime}\left(c_{1}, A\right)$.

Remark 2.19. We note in particular that in Lemma 2.17 (1), all that we need in order to make an analogous statement for a metric graph bundle is that $\phi(t) \geq M_{c_{1}}$, for all $t$.

Remark 2.20. It is not a priori clear that if a metric bundle satisfies a flaring condition, then an approximating metric graph bundle does so too (though this does follow a posteriori from Theorem 4.3 and Proposition 5.8). One reason is that the flaring condition is defined for any two qi lifts of a geodesic segment in the base. However, geodesics in the base space of the approximating metric graph bundle need not come from a geodesic in the base space of the metric bundle.

Lemma 2.22 below addresses this issue and proves that conclusions similar to those of Lemma 2.17above remain true for the approximating metric graph bundle. This is the main reason for giving explicitly a proof of Lemma 2.17 here in the context of metric bundles rather than for metric graph bundles (Lemma 2.18).

Let $p: X^{\prime} \rightarrow B^{\prime}$ be an $(f, c, K)$-metric bundle satisfying $\left(M_{k}, \lambda_{k}, n_{k}\right)$-flaring for all $k \geq 1$ and let $\pi: X \rightarrow B$ be an approximating metric graph bundle as in Lemma 1.21 As in Lemma 1.21 we suppose that the maps $\psi_{X}, \psi_{B}$ are $K_{1}-$ quasiisometries. Let $\psi_{X^{\prime}}$ (resp. $\psi_{B^{\prime}}$ ) be a quasi-isometric coarse inverse of the map $\psi_{X}$ (resp. $\psi_{B}$ ) constructed as in the proof of Lemma 1.1 (2). We assume that these maps are inverses of $\psi_{X}, \psi_{B}$ when restricted to the vertex sets $\mathcal{V}(X)$ and $\mathcal{V}(B)$ respectively. Moreover, we assume that $\psi_{X^{\prime}}, \psi_{B^{\prime}}$ are $K_{1}$-quasi-isometries. Also we assume that the restrictions of $\psi_{X}$ and $\psi_{X^{\prime}}$ to horizontal spaces (cf. Lemma 1.19) are $K_{1}$-quasi-isometries.

Let $B$ be $\delta_{0}$-hyperbolic. Suppose further that for every $k$-qi section of the approximating metric graph bundle, we obtain a $k^{\prime}$-qi section of the original bundle. For convenience of exposition we suppress the dependence of the constants (defined in the following lemma) on the parameters $f, c, K, \delta_{0}$ etc.

Lemma 2.21. With notation as above, let $X_{1}, X_{2}$ be two $k$-qi sections of the approximating metric graph bundle and $A_{0} \geq 0$. Suppose $d_{h}\left(X_{1}, X_{2}\right) \leq A_{0}$ and let $A_{1}=K_{1} \cdot \max \left\{A_{0}+K_{1}+1, M_{k^{\prime}}+K_{1}\right\}$. Then the following hold.

(1) For $A \geq A_{1}, U_{A}\left(X_{1}, X_{2}\right)$ is $K_{[2.21]}=K_{[2.21}(k, A)$-quasi-convex in $B$.

(2) Suppose $d_{u}\left(F_{u} \cap X_{1}, F_{u} \cap X_{2}\right)=C \geq A$ for some $u \in \mathcal{V}(B)$. Then $d_{B}\left(u, U_{A}\left(X_{1}, X_{2}\right)\right) \leq$

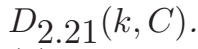

(3) Suppose $d_{h}\left(X_{1}, X_{2}\right) \geq K_{1}\left(M_{k^{\prime}}+K_{1}\right)$. Then the diameter of the set $U_{A}\left(X_{1}, X_{2}\right)$ is at most $D_{2.21}^{\prime}(k, A)$.

Proof. For the proof of this lemma we introduce the notation $d_{u}^{\prime}$ for the path metric on $X_{u}^{\prime}$ induced from $X^{\prime}$. Also let $A_{2}=A_{1} / K_{1}-1$.

(1) By Proposition 2.12 we have two $k^{\prime}$-qi sections $X_{1}^{\prime}, X_{2}^{\prime}$ of the metric bundle $X^{\prime}$ (corresponding to $X_{1}, X_{2}$ respectively) where $k^{\prime}=K_{2.12}\left(f, c, K, K_{1}, k\right)$. By choice of the constant $A_{1}$, we know that $U:=U_{A_{2}}\left(X_{1}^{\prime}, X_{2}^{\prime}\right)$ is a nonempty $K^{\prime}:=$ $K\left[2.17\left(k^{\prime}\right)\right.$-quasiconvex subset of $B^{\prime}$. Hence $\psi_{B^{\prime}}(U) \subset B$ is $D:=\left\{K_{1} \cdot K^{\prime}+K_{1}+\right.$ $D\left[1.26\left(\lambda, K_{1}\right)\right\}$-quasiconvex. Also note that $\psi_{B^{\prime}}(U) \subset U_{A}\left(X_{1}, X_{2}\right)$.

Now suppose $u \in\left(U_{A}\left(X_{1}, X_{2}\right) \backslash \psi_{B^{\prime}}(U)\right)$ is a point of $\mathcal{V}(B)$. Then $d_{u}^{\prime}\left(X_{1}^{\prime} \cap\right.$ $\left.F_{u}, X_{2}^{\prime} \cap F_{u}\right) \leq K_{1} A+K_{1}$. It follows that either $u \in U$ or $d_{B^{\prime}}(u, U) \leq D_{1}=$ 
$D 2.17\left(k^{\prime},\left(A . K_{1}+K_{1}\right) / A_{2}\right)$ (by Lemma $\left.2.17(1)\right)$. In any case, $d_{B}\left(u, \psi_{B^{\prime}}(U)\right) \leq$ $D_{2}=K_{1} D_{1}+K_{1}$. Hence, $\psi_{B^{\prime}}(U) \subset U_{A}\left(X_{1}, X_{2}\right) \subset N_{D_{2}}\left(\psi_{B^{\prime}}(U)\right)$. Since $\psi_{B^{\prime}}(U)$ is a $D$-quasi-convex set and since $B$ is $\lambda$-hyperbolic, it follows that $U_{A}\left(X_{1}, X_{2}\right)$ is $K\left[2.21\left(=\left(2 \lambda+D+D_{2}\right)\right)\right.$-quasi-convex.

(2) If $u \in U$ then we set $D \overline{2.21}(k, C)=0$. Otherwise $d_{u}^{\prime}\left(X_{1}^{\prime} \cap F_{u}, X_{2}^{\prime} \cap\right.$ $\left.F_{u}\right) \leq C . K_{1}+K_{1}$ since the restriction of the map $\psi_{X}$ to the horizontal space $F_{u}$ is a $K_{1}$-quasi-isometry. Hence by Lemma $2.17(1)$ we have $d_{B^{\prime}}(u, U) \leq D_{3}=$ $D \overline{2.17}\left(k^{\prime},\left(C . K_{1}+K_{1}\right) / A_{2}\right)$. Using the fact that $\psi_{B^{\prime}}$ is a $K_{1}$-quasi-isometry, we have $d_{B}\left(u, \psi_{B^{\prime}}(U)\right) \leq K_{1} \cdot D_{3}+K_{1}$. Hence $d\left(u, U_{A}\left(X_{1}, X_{2}\right)\right) \leq K_{1} D_{3}+K_{1}$. Set $D \overline{2.21}(k, C)=K_{1}+K_{1} \cdot D \overline{2.17}\left(k^{\prime}, A_{3}\right)$ where $A_{3}=\max \left\{1,\left(C . K_{1}+K_{1}\right) / A_{2}\right\}$.

(3) By the given condition, for all $z \in U, d_{z}^{\prime}\left(X_{1}^{\prime} \cap F_{z}, X_{2}^{\prime} \cap F_{z}\right) \geq M_{k^{\prime}}$ and so we can apply the flaring condition. Now let $b_{1}, b_{2} \in U$ and let $b \in\left[b_{1}, b_{2}\right]$; then $d_{b}^{\prime}\left(X_{1}^{\prime} \cap F_{b}, X_{2}^{\prime} \cap F_{b}\right) \leq \mu_{k^{\prime}}\left(K\left[2.17\left(k^{\prime}\right)\right)=D_{4}\right.$, say, by Corollary 1.16,

Finally, as noted in Remark [2.19] what we really used in the proof of Lemma 2.17(1) is the fact that the value of the function $\phi$ is always greater than or equal to $M_{k^{\prime}}$. Thus in the same way we have $d_{B^{\prime}}\left(b_{1}, b_{2}\right) \leq D \overline{2.17}\left(k^{\prime}, D_{4} / A_{2}\right)$. Taking

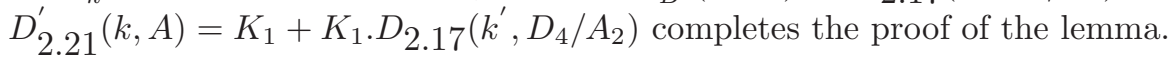

We unify the content of the last two lemmas in the following lemma in the form that shall use later.

Lemma 2.22. Given a function $f: \mathbb{N} \rightarrow \mathbb{N}, c_{1} \geq 1$ and $A_{0} \geq 0$, there exist $A_{2.22}^{\prime}=$ $A_{2.22}^{\prime}\left(f, c_{1}, A_{0}\right) \geq A_{0}, A_{2.22}^{\prime \prime}=A_{2.22}^{\prime \prime}\left(f, c_{1}\right)$ and three functions $K_{2.22}, \frac{2.22}{2.22}$ :

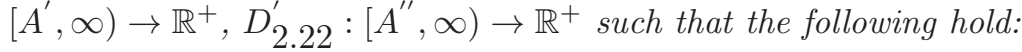

Suppose $X$ is an $(f, K)$-metric graph bundle over $B$ such that

1. either it satisfies a flaring condition

2. or it is an approximating metric graph bundle of a metric bundle that satisfies a flaring condition.

Suppose $B$ is a hyperbolic metric space. Let $C\left(X_{1}, X_{2}\right)$ be a ladder formed by two $c_{1}$-qi sections $X_{1}, X_{2}$. Let $d_{h}\left(X_{1}, X_{2}\right) \leq A_{0}$.

(1) If $A \geq A_{2.22}^{\prime}$ then $U_{A}\left(X_{1}, X_{2}\right)$ is $K_{2.22}(A)$-quasi-convex. Suppose $d_{u}\left(F_{u} \cap\right.$ $\left.X_{1}, F_{u} \cap X_{2}\right)=C \geq A$ for some $u \in \mathcal{V}(B)$. Then $d\left(u, U_{A}\left(X_{1}, X_{2}\right)\right) \leq$ $D \overline{2.22}(C)$.

(2) If $d_{h}\left(X_{1}, X_{2}\right) \geq A_{2.22}^{\prime \prime}$ then the diameter of the set $U_{A}\left(X_{1}, X_{2}\right)$ is at most $D_{2.22}^{\prime}(A)$.

The dependence of the functions $K_{[2.22}, D 2.22, D \frac{1}{2.22}$ on $c_{1}$, (which is implicit here) will be made explicit in the next section. Also we shall suppress the dependence of $A_{2.22}^{\prime} A_{2.22}^{\prime \prime}$ on $f$.

\section{Construction of Hyperbolic Ladders}

In this section we prove the main technical result leading to the combination theorem 4.3. A brief sketch follows: For a metric bundle, we first replace it with its approximating metric graph bundle. Then we work exclusively with metric graph bundles. In section 3.2 we prove that, under suitable hypotheses, ladders in a metric graph bundle are hyperbolic metric spaces when the metric graph bundle satisfies the properties of Lemma 2.22. To achieve this we first prove this result in section 
3.1 when the ladder is of small girth. Then, to prove hyperbolicity in the general case, a ladder is decomposed into small-girth ladders using qi sections. This gives a finite sequence of hyperbolic metric spaces and we check that the conditions of Corollary 1.52 are satisfied.

Notation and conventions: We fix the following notation and conventions to be used till the end of section 4. For us $p: X \rightarrow B$ will be either an $f$-metric graph bundle satisfying a flaring condition, or an approximating $(f-)$ metric graph bundle obtained from a metric bundle satisfying a flaring condition.

The symbols $g, \mu_{k}$ will have the same connotation as in Lemma 1.13 and Corollary 1.14 respectively. We shall assume that $B$ is $\delta$-hyperbolic and each of the horizontal spaces $F_{b}$ is $\delta^{\prime}$-hyperbolic for all vertices $b$ of $B$. We assume that the barycenter maps $\partial^{3} F_{b} \rightarrow F_{b}$ are (uniformly) coarsely surjective. Thus by Proposition 2.10 we know that the metric graph bundle admits a uniform $\left(K_{0}\right.$, say)qi section through any point of $X$. Lastly, often the dependence on these functions and constants will not be explicitly stated if it is clear from context. By points in a graph we shall always mean vertices, unless otherwise specified.

Lemma 3.1. For all $c_{1} \geq 1$, there exists $C_{3.1]}\left(=C_{3.1}\left(c_{1}\right)\right)$ such that the following holds.

Suppose $X_{1}$ and $X_{2}$ are two $c_{1}$-qi sections. Then through each point $x \in C\left(X_{1}, X_{2}\right)$ there exists a 93.1 qi section contained in $C\left(X_{1}, X_{2}\right)$.

Proof. We already know that there is a $K_{0}$-qi section, say $Y_{1}$, through $x$ in $X$. Now define a new section $Y_{2}$ as follows: let $Y_{2} \cap F_{b}$ be a nearest point projection, in the intrinsic metric on the horizontal space $F_{b}$, of $Y_{1} \cap F_{b}$ onto the horizontal geodesic $C\left(X_{1}, X_{2}\right) \cap F_{b}$. This defines a set theoretic section. We need to check that this is indeed a qi section. For this it is enough to check that $\forall b_{1}, b_{2} \in \mathcal{V}(B)$, with $d\left(b_{1}, b_{2}\right)=1$, the distance between $F_{b_{1}} \cap Y_{2}$ and $F_{b_{2}} \cap Y_{2}$ is uniformly bounded. This in turn follows immediately from Lemma 1.13, and Lemma 1.38 by choosing $C_{3.1}:=c^{\prime}+D \overline{1.38}\left(\delta^{\prime}, g\left(c^{\prime}\right)\right)$, where $c^{\prime}=2 \max \left\{K_{0}, c_{1}\right\}$.

The proof of the previous lemma parallels a construction of Mit98a. In our setting this can be stated as follows: Let $X_{1}, X_{2}$ be two $c_{1}$-qi-sections of a metric graph bundle $p: X \rightarrow B$, where each fiber (but not necessarily the base $B$ ) is uniformly $\delta$-hyperbolic. Let $C\left(X_{1}, X_{2}\right)$ be the associated ladder. By construction, $\lambda_{b}:=C\left(X_{1}, X_{2}\right) \cap F_{b}$ is a geodesic in the metric space $\left(F_{b}, d_{b}\right)$. Define $\pi_{b}: F_{b} \rightarrow \lambda_{b}$ as the nearest point projection of $F_{b}$ onto the geodesic $\lambda_{b}$ in the metric $d_{b}$. Let $\Pi_{X_{1}, X_{2}}: X \rightarrow C\left(X_{1}, X_{2}\right)$ be given by $\Pi_{X_{1}, X_{2}}(x)=\pi_{b}(x), \forall x \in F_{b}$. Extend $\Pi_{X_{1}, X_{2}}$ to all other edges in the usual way by sending the interior of an edge to the image of one of its end-points. The main technical theorem of Mit98a states

Theorem 3.2. Mit98a For $X, B, p$ as above, and $c_{1} \geq 1$, there exists $C \geq 1$ such that for two $c_{1}$-qi sections $X_{1}, X_{2}$, and $\forall x, y \in X$,

$$
d\left(\Pi_{X_{1}, X_{2}}(x), \Pi_{X_{1}, X_{2}}(y)\right) \leq C d(x, y)+C .
$$

Equivalently, $\Pi_{X_{1}, X_{2}}$ is a coarse Lipschitz retract of $X$ onto $C\left(X_{1}, X_{2}\right)$.

Simplification of Notation: We fix the following conventions and notation to be followed in the rest of this section. Fix $c_{1} \geq K_{0}$. Let $c_{i+1}=C_{3.1}^{i}\left(c_{1}\right)$, $i=1,2,3$ where $C_{3.1}^{i}$ is the $i$-th iterate of the function $C 3.1$. Note that if $Y$ is a $k$-qi section, and $k \leq c_{4}$, then it is also a $c_{4}$-qi section. We know that our metric 
graph bundle satisfies the bounded flaring condition. We shall denote the function $\mu_{c_{4}}$ (see Corollary 1.14) simply by $\mu$.

For two qi-sections $X_{1}, X_{2}$ in $X$ and $D \geq 0$ we shall denote by $C_{D}\left(X_{1}, X_{2}\right)$ the $D$-neighborhood of the ladder $C\left(X_{1}, X_{2}\right)$ in $X$.

From the definition of a ladder, we see that a ladder in a metric graph bundle is not connected. However, the first part of the next lemma says that a large enough neighborhood of a ladder is connected.

Lemma 3.3. Let $X_{1}, X_{2}$ be two $c_{1}-q i$-sections in $X$. Then

1) For any $D \geq 2 c_{1}, C_{D}\left(X_{1}, X_{2}\right)$ is connected.

2) Let $\gamma$ be a geodesic in $B$ and let $\tilde{\gamma}$ be its lift in $X_{1}$. Then, for any $D \geq 2 c_{1}$, $l(\tilde{\gamma}) \leq 2 c_{1} . l(\gamma)$ where $l(\tilde{\gamma})$ is the length computed in the D-neighborhood of the qi section $X_{1}$.

3) Let $X_{3}$ be a $c_{2}$-qi section lying inside $C\left(X_{1}, X_{2}\right)$. Then, for any $D \geq 2 c_{2}, X_{3}$ is the image of a $2 c_{2}$-Lipschitz map from $\mathcal{V}(B)$ into $C_{D}\left(X_{1}, X_{2}\right)$ equipped with the path metric induced from $X$. In particular, it is a $2 c_{2}$-qi section in $C_{D}\left(X_{1}, X_{2}\right)$.

Proof. Let $s: \mathcal{V}(B) \rightarrow X$ be a $c_{1}$-qi section and let $b_{1}, b_{2} \in \mathcal{V}(B)$ be adjacent vertices in $B$. Then $d\left(s\left(b_{1}\right), s\left(b_{2}\right)\right) \leq c_{1} \cdot d_{B}\left(b_{1}, b_{2}\right)+c_{1}=2 c_{1}$. Conclusion (1) follows. 2) follows from (1).

3) The first statement follows by taking $c_{2}$ in place of $c_{1}$ in (1). Since the projection map of the metric graph bundle $X$ to its base space $B$ is 1-Lipschitz by definition, it follows that a $c_{2}$-qi section lying inside $C\left(X_{1}, X_{2}\right)$ is a $2 c_{2}$-qi section inside $C_{D}\left(X_{1}, X_{2}\right)$, where the latter is equipped with the path metric induced from $X$.

3.1. Hyperbolicity of ladders: Special case. This subsection is devoted to proving the hyperbolicity of small girth ladders. Let $X_{1}, X_{2}$ be two $c_{1}$-qi sections in $X$ and let $d_{h}\left(X_{1}, X_{2}\right) \leq A_{0}$, say. Let $A:=A_{2.22}^{\prime}\left(c_{4}, A_{0}\right)$. We further assume, with reference to Lemma 2.22, that for any two $k$-qi sections $X_{3}, X_{4}, k \leq c_{4}$, lying inside the ladder $C\left(X_{1}, X_{2}\right)$, the set $U_{A}\left(X_{3}, X_{4}\right) \subset B$ is $K$-quasi-convex. We shall write simply $U\left(X_{3}, X_{4}\right)$ instead of $U_{A}\left(X_{3}, X_{4}\right)$ in what follows. Dependence of constants in the various lemmas and propositions below on the constants associated with the bundle will be implicit rather than explicit.

The rest of this subsection is devoted to proving the following:

Proposition 3.4. For all $L \geq 2 c_{4}$, and $c_{1}, A_{0}$ as above, there exist $\oint_{3.4}\left(=\oint_{3.4}\left(c_{1}, A_{0}, L\right)\right) \geq$ $0, K \sqrt{3.4}\left(=K K_{3.4}\left(c_{1}, A_{0}, L\right)\right) \geq 0$, D $3.4\left(=D \overline{3.4}\left(c_{1}, A_{0}, L\right)\right) \geq 0$ such that we have the following:

(1) $C_{L}\left(X_{1}, X_{2}\right)$ is $\overline{3.4}$-hyperbolic with the path metric induced from $X$, and $X_{1}, X_{2}$ are $K_{3.4}$ quasiconvex in $C_{L}\left(X_{1}, X_{2}\right)$.

(2) If $d_{h}\left(X_{1}, X_{2}\right) \geq A_{2.22}^{\prime \prime}\left(c_{1}\right)$, then $X_{1}, X_{2}$ are D 3.4 cobounded in $C_{L}\left(X_{1}, X_{2}\right)$.

Idea of the proof: The proof of this proposition is rather long. Therefore, we shall break it up into several lemmas. The idea is as follows. We define a set of discrete paths $c(x, y)$, one for each pair of points $x, y \in \mathcal{V}(X) \cap C\left(X_{1}, X_{2}\right)$ and check that they satisfy the three properties of Corollary 1.40 Given $x, y \in$ $\mathcal{V}(X) \cap C\left(X_{1}, X_{2}\right)$ first we construct two qi sections through them. Then, $c(x, y)$ consists of three parts: two of them are in the two sections containing $x, y$ and the other one is a horizontal geodesic of uniformly bounded length. Then any problem of length computation is transferred to the sections. For instance computing the 
Hausdorff distance between two paths or proving slimness of triangles becomes easy when we apply this strategy to the parts of the paths that already lie in a quasiisometric section of the hyperbolic base space $B$. Lemma 2.22 and the bounded flaring condition are the main tools of the proof.

We denote by $d^{\prime}$ the path metric on a neighborhood of a ladder induced from $X$. Also $H d^{\prime}$ will denote the Hausdorff distance between sets in a neighborhood of ladder and $H d_{B}$ will denote Hausdorff distance between sets in $B$.

Definition of path family: Let $x, y \in C\left(X_{1}, X_{2}\right)$ be two vertices. By Lemma 3.1 we can choose two $c_{2}$-qi sections $X_{3}$ and $X_{4}$ through $x$ and $y$ respectively in $C\left(X_{1}, X_{2}\right)$. Recall that $U\left(X_{3}, X_{4}\right) \subseteq \mathcal{V}(B)$ is a $K$-quasi-convex subset of $B$. Join $p(x)$ to $U\left(X_{3}, X_{4}\right)$ by a shortest geodesic $\gamma_{x, y}$ in $B$ ending at $b_{x, y} \in U\left(X_{3}, X_{4}\right)$. Let $\tilde{\gamma}_{x, y}$ be the lift of $\gamma_{x, y}$ in $X_{3}$, ending at $s_{x, y}$. Let $t_{x, y}$ be the lift of $b_{x, y}$ in $X_{4}$. We note that $d_{b_{x, y}}\left(t_{x, y}, s_{x, y}\right) \leq A$. Now let $\beta_{x, y}$ be a geodesic in $B$ joining $p(y)$ and $b_{x, y}$, and let $\tilde{\beta}_{x, y}$ be the lift of $\beta_{x, y}$ in $X_{4}$. We define $c(x, y)$ to be the union of the three paths: $\tilde{\gamma}_{x, y}, \tilde{\beta}_{x, y}$ and the sequence of consecutive vertices on the geodesic segment $F_{b_{x, y}} \cap C\left(X_{1}, X_{2}\right)$ between $t_{x, y}$ and $s_{x, y}$. We see that there is an asymmetry in the definition of $c(x, y)$ and a number of choices are involved. However, for each unordered pair $\{x, y\}$ make the choices once and for all and choose either $c(x, y)$ or $c(y, x)$ as the path joining the points $x, y$. (See figure below.)

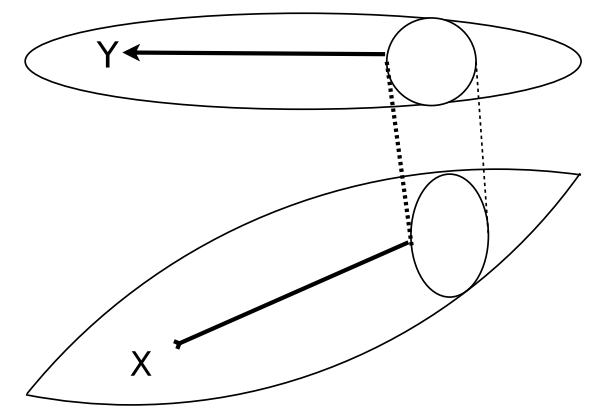

Path families: Special case

Lemma 3.5. Given $D_{1} \geq 0$ there exist constants $D_{3.5}=D_{3.5}\left(c_{1}, A, D_{1}\right)$ and $D_{3.5}^{\prime}=D_{3.5}^{\prime}\left(c_{1}, A, D_{1}\right)$ such that the following holds:

Let $x, y \in \mathcal{V}(X) \cap C\left(X_{1}, X_{2}\right)$ with $d(x, y) \leq D_{1}$. Then, $d^{\prime}(x, y)$ - the distance between $x, y$ in the path metric on $C_{L}\left(X_{1}, X_{2}\right)$, is bounded by $D_{3.5}^{\prime}$. Moreover, the length of the path $c(x, y)$ is at most $D \overline{3.5}$.

Proof. Let $\tilde{y}$ be the lift of $p(x)$ in $X_{4}$. Since $p$ is a 1-Lipschitz map, $d_{B}(p(y), p(\tilde{y})) \leq$ $D_{1}$. Hence, $d(y, \tilde{y}) \leq c_{2} \cdot D_{1}+c_{2}$, since $X_{4}$ is a $c_{2}$-qi section. Therefore $d(\tilde{y}, x) \leq$ $c_{2} . D_{1}+D_{1}+c_{2}$. Then, since inclusions of the fibers of the map $p$ are uniformly metrically proper embeddings as measured by $f$, we have $d_{p(x)}(\tilde{y}, x) \leq f\left(c_{2} \cdot D_{1}+\right.$ $\left.D_{1}+c_{2}\right)$.

By Lemma $3.3(2), d^{\prime}(y, \tilde{y}) \leq 2 c_{2} \cdot D_{1}$. Thus $d^{\prime}(x, y) \leq 2 c_{2} \cdot D_{1}+f\left(c_{2} \cdot D_{1}+D_{1}+c_{2}\right)$ and the first part of the lemma is proved, with $D_{3.5}^{\prime}:=2 c_{2} \cdot D_{1}+f\left(c_{2} \cdot D_{1}+D_{1}+c_{2}\right)$.

Next by Lemma 2.22(2), we have

$$
d_{B}\left(p(x), b_{x, y}\right) \leq D_{1}^{\prime}:=D \overline{2.22}\left(c_{2}, \max \left\{A, f\left(c_{2} D_{1}+D_{1}+c_{2}\right)\right\}\right) .
$$


Thus $d_{B}\left(p(y), b_{x, y}\right) \leq D_{1}+D_{1}^{\prime}$. From this and Lemma 3.3(2), the second part of the lemma follows, with $D \overline{3.5}:=A+2 c_{2} \cdot D_{1}+4 c_{2} \cdot D_{1}^{\prime}$.

Remark 3.6. Note that in the first part of Lemma 3.5. we have not assumed that $C\left(X_{1}, X_{2}\right)$ is of small girth.

We next show that the path family is coarsely well-defined, i.e. ambiguities in the definition of the paths can be ignored. More precisely, the different choices of paths joining the same pair of points are at a uniformly bounded Hausdorff distance from each other.

Suppose $X_{3}, X_{3}^{\prime}$ are two $k$-qi sections in $C\left(X_{1}, X_{2}\right)$ containing $x$; and $X_{4}$ is a $k$-qi section containing $y$, where $k \leq c_{4}$. Consider the two paths $c(x, y)$ and $c^{\prime}(x, y)$ joining $x, y$ defined using $X_{3}, X_{4}$ and $X_{3}^{\prime}, X_{4}$ respectively (defined as before).

Let $V:=U\left(X_{1}, X_{2}\right), W:=U\left(X_{3}, X_{4}\right)$ and $W^{\prime}:=U\left(X_{3}^{\prime}, X_{4}\right)$. Then $V \subset W$, $V \subset W^{\prime}$. Join $p(x)$ to $V$ by a shortest geodesic $\gamma$ in $B$ and let $\tilde{\gamma}, \tilde{\gamma}^{\prime}$ be the lifts of $\gamma$ in $X_{3}$ and $X_{3}^{\prime}$ respectively. Similarly join $p(x)$ to $W, W^{\prime}$ respectively by shortest geodesics $\gamma_{x, y}$ and $\gamma_{x, y}^{\prime}$ and let $\tilde{\gamma}_{x, y}$ and $\tilde{\gamma}_{x, y}^{\prime}$ be their lifts in $X_{3}$ and $X_{3}^{\prime}$ respectively. Let $s_{x, y}, s_{x, y}^{\prime}$ be the end points of $\tilde{\gamma}_{x, y}, \tilde{\gamma}_{x, y}^{\prime}$ respectively, and let $b_{x, y}, b_{x, y}^{\prime}$ be the end points of $\gamma_{x, y}$ and $\gamma_{x, y}^{\prime}$.

Lemma 3.7. With notation (in particular $k, A$ ) as above, there exists $D \overline{3.7}(=$ $D \overline{3.7}(k, A))$ such that $d_{B}\left(b_{x, y}, b_{x, y}^{\prime}\right)$ is bounded by $D \overline{3.7}$.

Proof. Note that $V \subset W \cap W^{\prime}$, and that $V, W, W^{\prime}$ are all $K$-quasiconvex subsets of $B$. Therefore, by Lemma $1.31(2)$, concatenating $\gamma_{x, y}\left(\right.$ resp. $\left.\gamma_{x, y}^{\prime}\right)$ with a geodesic joining $b_{x, y}$ (resp. $b_{x, y}^{\prime}$ ) to the terminal point of $\gamma$, we obtain $(3+2 K)$-quasigeodesics. These quasi-geodesics have the same end points as those of $\gamma$. Since $B$ is a $\delta$-hyperbolic graph, by Lemma 1.26 we can find $b, b^{\prime} \in \gamma \cap \mathcal{V}(B)$, such that $d_{B}\left(b_{x, y}, b\right) \leq D_{3}, d_{B}\left(b_{x, y}^{\prime}, b^{\prime}\right) \leq D_{3}$, where $D_{3}:=D \overline{1.26}(\delta, 3+2 K)$. If $b \in$ $\left[p(x), b^{\prime}\right] \subset \gamma$ then $b_{x, y} \in N_{2 . D_{3}+\delta}\left(\gamma_{x, y}^{\prime}\right)$. Otherwise, $b^{\prime} \in[p(x), b]$, so that $b_{x, y}^{\prime} \in$ $N_{2 . D_{3}+\delta}\left(\gamma_{x, y}\right)$. Without loss of generality, let us assume that $b \in\left[p(x), b^{\prime}\right]$.

The end points of $\gamma$ are in $U\left(X_{3}, X_{3}^{\prime}\right)$ which is a $K$-quasi-convex set in $B$. Hence by the bounded flaring condition (Corollary 1.14), we know that for all points $b_{2} \in \gamma$, $d_{b_{2}}\left(X_{3} \cap F_{b_{2}}, X_{3}^{\prime} \cap F_{b_{2}}\right) \leq A . \mu(K)$. In particular, $d_{b}\left(X_{3} \cap F_{b}, X_{3}^{\prime} \cap F_{b}\right) \leq A . \mu(K)$. Similarly, $d_{b}\left(X_{3} \cap F_{b}, X_{4} \cap F_{b}\right) \leq A . \mu\left(D_{3}\right)$. Thus,

$$
\begin{aligned}
d_{b}\left(X_{3}^{\prime} \cap F_{b}, X_{4} \cap F_{b}\right) & \leq d_{b}\left(X_{3} \cap F_{b}, X_{3}^{\prime} \cap F_{b}\right)+d_{b}\left(X_{3} \cap F_{b}, X_{4} \cap F_{b}\right) \\
& \leq A . \mu(K)+A . \mu\left(D_{3}\right) .
\end{aligned}
$$

We know that $\left[p(x), b^{\prime}\right] \subset N_{\delta+D_{3}}\left(\gamma_{x, y}^{\prime}\right)$. Let $b_{1}^{\prime} \in \gamma_{x, y}^{\prime} \cap \mathcal{V}(B)$ be such that $d_{B}\left(b, b_{1}^{\prime}\right) \leq \delta+D_{3}$. Then $d_{b_{1}^{\prime}}\left(X_{3}^{\prime} \cap F_{b_{1}^{\prime}}, X_{4} \cap F_{b_{1}^{\prime}}\right) \leq \mu\left(\delta+D_{3}\right) \cdot \max \left\{d_{b}\left(X_{3}^{\prime} \cap\right.\right.$ $\left.\left.F_{b}, X_{4} \cap F_{b}\right), 1\right\}$ and hence $d_{b_{1}^{\prime}}\left(X_{3}^{\prime} \cap F_{b_{1}^{\prime}}, X_{4} \cap F_{b_{1}^{\prime}}\right) \leq A . \mu\left(\delta+D_{3}\right)\left\{\mu\left(D_{3}\right)+\mu(K)\right\}$.

Denoting the right hand side of the preceding inequality by $D^{\prime}$, we have, by Lemma $2.22(1), d_{B}\left(b_{1}^{\prime}, b_{x, y}^{\prime}\right) \leq D 2 \overline{2.22}\left(k, D^{\prime}\right)$. Since, $d_{B}\left(b_{x, y}, b_{x, y}^{\prime}\right) \leq d_{B}\left(b_{x, y}, b\right)+$ $d_{B}\left(b, b_{1}^{\prime}\right)+d_{B}\left(b_{1}^{\prime}, b_{x, y}^{\prime}\right)$, therefore

$$
\left.d_{B}\left(b_{x, y}, b_{x, y}^{\prime}\right) \leq D_{3}+\left(\delta+D_{3}\right)+D \overline{2.22}{ }^{\left(k, D^{\prime}\right.}\right)=\delta+2 D_{3}+D \overline{2.22}^{\left(k, D^{\prime}\right) .}
$$

Taking $D \overline{3_{3.7}}:=\delta+2 D_{3}+D \overline{2.22}\left(k, D^{\prime}\right)$ completes the proof of the lemma. 
Lemma 3.8. With $k, A$ as above there exists $D_{3.8}\left(=D_{3.8}(k, A)\right)$ such that the Hausdorff distance between $c(x, y)$ and $c^{\prime}(x, y)$ is bounded by $D \overline{3.8}$.

Proof. Step 1: By Lemma 3.7 we have $d_{B}\left(b_{x, y}, b_{x, y}^{\prime}\right) \leq D_{3.7}(k, A)$. Hence, by $\delta$-hyperbolicity of $B, H d_{B}\left(\beta_{x, y}, \beta_{x, y}^{\prime}\right) \leq \delta+D \overline{3.7}(k, A)$. Since $X_{4}$ is a $k$-qi section, we have, by Lemma $3.3(2)$,

$$
H d^{\prime}\left(\tilde{\beta}_{x, y}, \tilde{\beta}_{x, y}^{\prime}\right) \leq 2 k \cdot(\delta+D \overline{3.7}(k, A)) .
$$

Step 2: Similarly,

$$
H d^{\prime}\left(\left[s_{x, y}, t_{x, y}\right],\left[s_{x, y}^{\prime}, t_{x, y}^{\prime}\right]\right) \leq A+2 k \cdot D \overline{3.7}(k, A) .
$$

where $\left[s_{x, y}, t_{x, y}\right],\left[s_{x, y}^{\prime}, t_{x, y}^{\prime}\right]$ are the horizontal geodesic segments of $c(x, y)$ and $c^{\prime}(x, y)$ respectively, each of length at most $A$.

Step 3: Now we calculate the Hausdorff distance between $\tilde{\gamma}_{x, y}$ and $\tilde{\gamma}_{x, y}^{\prime}$. Let $\tilde{\gamma}_{x, y}^{\prime \prime}$ be the lift of $\gamma_{x, y}$ in $X_{3}^{\prime}$. Then, as in Step 1, we have $H d^{\prime}\left(\tilde{\gamma}_{x, y}^{\prime}, \tilde{\gamma}_{x, y}^{\prime \prime}\right) \leq$ $2 k .(\delta+D \overline{3.7}(k, A))$. Since $\gamma$ joins two points of $U\left(X_{3}, X_{3}^{\prime}\right)$ which is $K$-quasi-convex in $B$, it follows that $d_{b_{2}}\left(X_{3} \cap F_{b_{2}}, X_{3}^{\prime} \cap F_{b_{2}}\right) \leq A . \mu(K)$ for all points $b_{2} \in \gamma$ by the bounded flaring condition. Since there is a point $b \in \gamma$ such that $d_{B}\left(b, b_{x, y}\right) \leq$ $D_{3}:=D_{1.26}(\delta, 3+2 K)$, we have the following using the boundedness of the flaring condition again:

$$
\begin{aligned}
d_{b_{x, y}}\left(X_{3} \cap F_{b_{2}}, X_{3}^{\prime} \cap F_{b_{2}}\right) & \leq \mu\left(D_{3}\right) \cdot \max \left\{d_{b}\left(X_{3} \cap F_{b}, X_{3}^{\prime} \cap F_{b}\right), 1\right\} \\
& \leq A \cdot \mu\left(D_{3}\right) \cdot \mu(K) .
\end{aligned}
$$

Let $A_{1}=A \cdot \mu\left(D_{3}\right) \cdot \mu(K)$; then $U_{A_{1}}\left(X_{3}, X_{3}^{\prime}\right)$ is $K^{\prime}:=K\left[2.22\left(c_{2}, A_{1}\right)\right.$-quasi-convex. Note that $\gamma_{x, y}$ joins two points of $U_{A_{1}}\left(X_{3}, X_{3}^{\prime}\right)$. Therefore, by Lemma 2.22 (1) and the bounded flaring condition, we have for all $b_{1} \in \gamma_{x, y}, d_{b_{1}}\left(X_{3} \cap F_{b_{1}}, X_{3}^{\prime} \cap F_{b_{1}}\right) \leq$ $\mu\left(K^{\prime}\right) \cdot A_{1}$. Hence $H d^{\prime}\left(\tilde{\gamma}_{x, y}, \tilde{\gamma}_{x, y}^{\prime \prime}\right) \leq \mu\left(K^{\prime}\right) \cdot A_{1}$, and therefore

$$
\begin{aligned}
H d^{\prime}\left(\tilde{\gamma}_{x, y}, \tilde{\gamma}_{x, y}^{\prime}\right) & \leq H d^{\prime}\left(\tilde{\gamma}_{x, y}, \tilde{\gamma}_{x, y}^{\prime \prime}\right)+H d^{\prime}\left(\tilde{\gamma}_{x, y}^{\prime}, \tilde{\gamma}_{x, y}^{\prime \prime}\right) \\
& \leq \mu\left(K^{\prime}\right) \cdot A_{1}+2 k \cdot(\delta+D \underline{3.7}(k, A)) .
\end{aligned}
$$

Finally, since

$$
\begin{aligned}
& H d^{\prime}\left(c(x, y), c^{\prime}(x, y)\right) \\
& \leq \max \left\{H d^{\prime}\left(\tilde{\beta}_{x, y}, \tilde{\beta}_{x, y}^{\prime}\right), H d^{\prime}\left(\left[s_{x, y}, t_{x, y}\right],\left[s_{x, y}^{\prime}, t_{x, y}^{\prime}\right]\right), H d^{\prime}\left(\tilde{\gamma}_{x, y}, \tilde{\gamma}_{x, y}^{\prime}\right)\right\},
\end{aligned}
$$

the lemma follows, taking $D \overline{3.8}:=\mu\left(K^{\prime}\right) \cdot A_{1}+2 k \cdot(\delta+D \overline{3.7}(k, A))$.

Lemma 3.9. With notation (in particular $k, A$ ) as above, there exists $D \overline{3.9}(=$ $D \overline{3.9}(k, A))$ such that if $c(x, y), c(y, x)$ are defined using two $k$-qi sections $X_{3}, X_{4}$ where $k \leq c_{4}, x \in X_{3}$ and $y \in X_{4}$, then $\operatorname{Hd}(c(x, y), c(y, x))$ is bounded by $D \overline{3.9}$.

Proof. Let $\alpha$ be a geodesic in $B$ joining $b_{x, y}$ and $b_{y, x}$. Since $\alpha$ joins two points of $U\left(X_{3}, X_{4}\right)$ which is a $K$-quasiconvex subset of $B$, we have:

i) $d_{b}\left(F_{b} \cap X_{3}, F_{b} \cap X_{4}\right) \leq \mu(K) A$ for all $b \in \alpha$, by the bounded flaring condition for metric graph bundles.

ii) $\gamma_{x, y} \cup \alpha$ is a $(3+2 K)$-quasi-geodesic by Lemma 1.31 (2). Hence, $H d_{B}\left(\gamma_{x, y} \cup\right.$ $\left.\alpha,\left[p(x), b_{y, x}\right]\right) \leq D \overline{1.26}(\delta, 3+2 K)$, by Lemma 1.26. Similarly, $H d_{B}\left(\gamma_{y, x} \cup \alpha,\left[p(y), b_{x, y}\right]\right) \leq$ $D_{1.26}(\delta, 3+2 K)$. 
Therefore, for all $z \in \tilde{\gamma}_{y, x}, p(z)$ is in the ${ }_{1.26}(\delta, 3+2 K)$-neighborhood of $\left[p(y), b_{x, y}\right]$. Thus $z$ is contained in the $2 k \cdot D \overline{1.26}(\delta, 3+2 K)$-neighborhood of $\tilde{\beta}_{x, y} \subset$ $c(x, y)$ using the fact that $X_{4}$ is a $k$-qi section and Lemma $3.3(2)$.

Again for all $z \in F_{b_{y, x}} \cap \mathcal{V}\left(C\left(X_{1}, X_{2}\right)\right), d_{b_{y, x}}\left(z, s_{y, x}\right) \leq A$. It follows that in this case $z$ is contained in the $\left(A+2 k \cdot D_{1.26}(\delta, 3+2 K)\right)$-neighborhood of $c(x, y)$.

Now, suppose $z \in \tilde{\beta}_{y, x}$. Since $\frac{B}{B}$ is $\delta$-hyperbolic, $p(z) \in N_{\delta}\left(\gamma_{x, y} \cup \alpha\right)$. If $p(z) \in N_{\delta}\left(\gamma_{x, y}\right)$ then $z$ is contained in the $2 k$. $\delta$-neighborhood of $\tilde{\gamma}_{x, y} \subset c(x, y)$. Otherwise, $p(z) \in N_{\delta}(\alpha)$. Suppose $b_{1} \in \alpha$ such that $d_{B}\left(p(z), b_{1}\right) \leq \delta$. As in the first paragraph of the proof we have $d_{b_{1}}\left(F_{b_{1}} \cap X_{3}, F_{b_{1}} \cap X_{4}\right) \leq \mu(K) A$. Using the fact that $H d_{B}\left(\gamma_{x, y} \cup \alpha,\left[p(x), b_{y, x}\right]\right) \leq D \overline{1.26}(\delta, 3+2 K)$ we see that $z$ is contained in the $(\mu(K) A+2 k(\delta+D \overline{1.26}(\delta, 3+2 K)))$-neighborhood of $\tilde{\beta}_{x, y} \subset c(x, y)$.

It follows that $c(y, x)$ is contained in the $(\mu(K) A+2 k(\delta+D \overline{1.26}(\delta, 3+2 K)))$ neighborhood of $c(x, y)$. Similarly it follows that $c(x, y)$ is contained in the $(\mu(K) A+$ $2 k(\delta+D \overline{1.26}(\delta, 3+2 K)))$-neighborhood of $c(y, x)$. Hence $H d^{\prime}(c(x, y), c(y, x)) \leq$ $D_{3.9}:=\mu(K) A+2 k\left(\delta+D_{1.26}(\delta, 3+2 K)\right)$.

Corollary 3.10. With notation (in particular $k, A$ ) as above, there exists $D \overline{3.10}(=$ $D \overline{3.10}(k, A))$ such that the following holds.

Let $x, y \in C\left(X_{1}, X_{2}\right)$. Then the Hausdorff distance between any pair of paths joining $x, y$ defined in the same way as $c(x, y)$ using $k$-qi sections passing through $x, y$, is at most $D \overline{3.10}$.

Proof. Choose $D \overline{3.10}:=2(D \overline{3.8}+D(\overline{3.9})$.

Lemma 3.11. With notation (in particular $k, A$ ) as above, there exists $D \overline{3.11}(=$ $D \overline{3.11}(k, A))$ such that the following holds.

Suppose $X_{3}, X_{4}, X_{5}$ are $k$-qi sections in $C\left(X_{1}, X_{2}\right)$ such that $z \in X_{5}, y \in X_{4} \subset$ $C\left(X_{1}, X_{5}\right) \subset C\left(X_{1}, X_{2}\right)$ and $x \in X_{3} \subset C\left(X_{1}, X_{4}\right) \subset C\left(X_{1}, X_{2}\right)$. Then the triangle formed by the paths $c(x, y), c(y, z), c(x, z)$, defined using the pairs $X_{3}, X_{4} ; X_{4}, X_{5}$ and $X_{3}, X_{5}$ respectively, is 233.11 -slim.

Proof. We have $U\left(X_{3}, X_{5}\right) \subset U\left(X_{4}, X_{5}\right) \cap U\left(X_{3}, X_{4}\right)$ and we know that all of these three sets are $K$-quasi-convex in $B$.

Case 1: Suppose $x, y$ are in the same horizontal space and $d_{p(x)}(x, y) \leq A$.

Then $p(x) \in U\left(X_{3}, X_{4}\right)$. Since $\gamma_{x, z}$ ends in $U\left(X_{3}, X_{5}\right) \subset U\left(X_{3}, X_{4}\right)$, it joins two points of $U\left(X_{3}, X_{4}\right)$ which we know is $K$-quasi-convex. Hence, by Corollary 1.14 we have for all $b^{\prime} \in \gamma_{x, z}, d_{b^{\prime}}\left(X_{3} \cap F_{b^{\prime}}, X_{4} \cap F_{b^{\prime}}\right) \leq A . \mu(K)$.

Now we show that $d_{B}\left(b_{x, z}, b_{y, z}\right)$ is small. Recall that $b_{x, z} \in U\left(X_{3}, X_{5}\right) \subset$ $U\left(X_{4}, X_{5}\right)$ and $b_{y, z} \in U\left(X_{4}, X_{5}\right)$. Thus $\gamma_{y, z} \cup\left[b_{y, z}, b_{x, z}\right]$ is a $(3+2 K)$-quasi-geodesic in $B$, by Lemma $1.31(2)$. Hence, there is a point $b_{2} \in \gamma_{x, z}$, such that $d_{B}\left(b_{y, z}, b_{2}\right) \leq$ $D_{1.26}(\delta, 3+2 K)$, by Lemma 1.26. Since $d_{b_{y, z}}\left(F_{b_{y, z}} \cap X_{4}, F_{b_{y, z}} \cap X_{5}\right) \leq A$, we have by bounded flaring, $d_{b_{2}}\left(F_{b_{2}} \cap X_{4}, F_{b_{2}} \cap X_{5}\right) \leq A . \mu(D \overline{1.26}(\delta, 3+2 K))$. Therefore, $d_{b_{2}}\left(F_{b_{2}} \cap X_{3}, F_{b_{2}} \cap X_{5}\right)=d_{b_{2}}\left(F_{b_{2}} \cap X_{3}, F_{b_{2}} \cap X_{4}\right)+d_{b_{2}}\left(F_{b_{2}} \cap X_{4}, F_{b_{2}} \cap X_{5}\right) \leq$ $A .\{\mu(K)+\mu(D \overline{1.26}(\delta, 3+2 K)\}$. Now, by Lemma 2.22 (1), we get

$$
d_{B}\left(b_{2}, b_{x, z}\right) \leq D \overline{2.22}\left(k, A \cdot \mu(K)+A \cdot \mu\left(D_{\overline{1.26}}(\delta, 3+2 K)\right) .\right.
$$

Hence

$$
d_{B}\left(b_{x, z}, b_{y, z}\right) \leq D \overline{1.26}(\delta, 3+2 K)+D \overline{2.22}(k, A \cdot \mu(K)+A \cdot \mu(D \overline{1.26}(\delta, 3))) .
$$


It follows by arguments similar to that of Lemma 3.8 that

$$
H d^{\prime}(c(x, z), c(y, z)) \leq A \cdot \mu(K)+2 k \cdot\left(\delta+d_{B}\left(b_{x, z}, b_{y, z}\right)\right) .
$$

Replacing $d_{B}\left(b_{x, z}, b_{y, z}\right)$ in the right hand expression with its upper bound obtained above, we get a constant $D_{3.11}^{\prime}$. Hence the lemma follows in this case by choosing $D \overline{3.11} \geq D^{\prime}$

Case 2: We consider the general case. For the rest of the proof, we shall assume that all the paths of the form $c(u, v)\left(u, v \in X_{3} \cup X_{4} \cup X_{5}\right)$, are constructed using the sections $X_{3}, X_{4}, X_{5}$ only, unless otherwise specified. We first show that $H d^{\prime}\left(c(x, z), \tilde{\gamma}_{x, y} \cup c\left(s_{x, y}, z\right)\right)$ is bounded by a constant depending on $k$ and $A$.

Let $\bar{b}$ be a nearest point projection of $b_{x, y}$ on $U\left(X_{3}, X_{5}\right)$. By Lemma 1.32, $d_{B}\left(\bar{b}, b_{x, z}\right) \leq D_{1.32}=D_{1.32}(\delta, K)$. Let $\gamma_{2}$ be a geodesic joining $b_{x, y}$ to $\bar{b}$ and let $\widetilde{\gamma_{2}}$ be a lift of $\gamma_{2}$ in $X_{3}$. Note that $\gamma_{x, y} \cup \gamma_{2}$ is a $(3+2 K)$-quasi geodesic in $B$. Thus the Hausdorff distance between $\gamma_{x, z}$ and $\gamma_{x, y} \cup \gamma_{2}$ is at most $\delta+D \overline{1.32}+D \overline{1.26}(\delta, 3+2 K)$. Hence the Hausdorff distance between $\tilde{\gamma}_{x, y} \cup c\left(s_{x, y}, z\right)$ and $c(x, z)$, in $C_{L}\left(X_{1}, X_{2}\right)$, is at most $2 k \cdot\{\delta+D \overline{1.32}+D \overline{1.26}(\delta, 3+2 K)\}+A=D_{1}$, say.

Again by case 1 , we know that $H d\left(c\left(s_{x, y}, z\right), c\left(t_{x, y}, z\right)\right) \leq D_{\overline{3.11}}^{\prime}$ Hence, $H d\left(c(x, z), \tilde{\gamma}_{x, y} \cup\right.$ $\left.\left[s_{x, y}, t_{x, y}\right] \cup c\left(t_{x, y}, z\right)\right)$ is at most $A+D_{1}+D_{3.11}^{\prime}$ Also, if we define the paths $c\left(z, t_{x, y}\right), c(z, y)$ with respect to the sections $\bar{X}_{4}, X_{5}$ by taking $\gamma_{z, t_{x, y}}=\gamma_{z, y}$, the triangle formed by the paths $c\left(z, t_{x, y}\right), c(z, y)$ and $\tilde{\beta}_{y, t_{x, y}}$ is $2 k \delta$-slim.

Thus by Corollary 3.10 , the triangle formed by the paths $\tilde{\beta}_{y, t_{x, y}}, c\left(t_{x, y}, z\right)$ and $c(y, z)$ is $D_{2}$-slim where $D_{2}=2 k . \delta+2 . D 3.10$. Taking $D \overline{3.11}:=A+D_{1}+D_{3.11}^{\prime}+D_{2}$, the lemma follows.

\section{Proof of Proposition 3.4}

We verify that the set of paths $\{c(x, y)\}$ defined earlier in this section satisfies the properties of Corollary 1.40. Then, as per the notation of Corollary 1.40, let $D=L, C_{1}:=2 c_{2}, \Phi(N)=D \overline{3.5}\left(c_{2}, A, N\right)$ and $C_{2}=D 3.11\left(c_{4}, A\right)+2 . D \overline{3.10}\left(c_{4}, A\right)$.

Proof of properties 1 and 2: These follow from Lemma [3.3)(2) and Lemma 3.5 respectively.

Proof of property 3: Suppose $x, y \in C\left(X_{1}, X_{2}\right)$. If $x^{\prime}, y^{\prime} \in c(x, y)$ then the segment of $c(x, y)$ between $x^{\prime}, y^{\prime}$, say $\left.c(x, y)\right|_{\left[x^{\prime}, y^{\prime}\right]}$, is a possible candidate for the definition of $c\left(x^{\prime}, y^{\prime}\right)$. Hence by Corollary 3.10 the Hausdorff distance of $\left.c(x, y)\right|_{\left[x^{\prime}, y^{\prime}\right]}$ and $c\left(x^{\prime}, y^{\prime}\right)$ is bounded by $D \overline{3.10}\left(c_{2}, A\right) \leq C_{2}$.

Proof of property 4: Let $x, y, z \in C\left(X_{1}, X_{2}\right)$. Then using Lemma 3.1 we may assume, without loss of generality, that $x, y, z$ are contained in three $c_{4}$-qi sections $X_{3}, X_{4}, X_{5}$ respectively, where $X_{4} \subseteq C\left(X_{1}, X_{5}\right), X_{3} \subseteq C\left(X_{1}, X_{4}\right)$. Now, the triangle formed by the paths $c(x, y), c(y, z), c(x, z)$ defined using these sections is $D 3.11\left(c_{4}, A\right)$-slim by Lemma 3.11. Hence, by Corollary 3.10, any triangle with vertices $x, y, z$ formed by such paths is $\left\{D \overline{3.11}\left(c_{4}, A\right)+2 . D \overline{3.10}\left(c_{4}, A\right)\right\}$-slim. It follows from Corollary 1.40 that $C_{L}\left(X_{1}, X_{2}\right)$ is $\delta 3.4$-hyperbolic for some $\delta 3.4 \geq 0$.

By Lemma $3.3(3)$, it follows that $X_{1}, X_{2}$ are the images of $2 c_{1}$-quasi-isometric embeddings of $B$ into the $\overline{3.4}$-hyperbolic metric space $C_{L}\left(X_{1}, X_{2}\right)$. Thus, they are

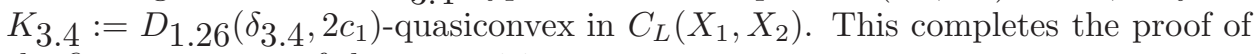
the first statement of the proposition. 
From the given conditions it follows by Lemma 2.22 (2) that $U\left(X_{1}, X_{2}\right)$ is bounded. Hence, for any $x \in X_{1}$ and $y \in X_{2}$ the $K[1.40$-quasi-geodesic $c(x, y)$ passes through the (uniformly) bounded set $p^{-1}\left(U\left(X_{1}, X_{2}\right)\right) \cap C\left(X_{1}, X_{2}\right)$ (by Corollary 1.40). Since $C_{L}\left(X_{1}, X_{2}\right)$ has been proven to be hyperbolic, stability of quasigeodesics (Lemma 1.26) completes the proof of the second statement of the proposition.

\subsection{Hyperbolicity of ladders: General case.}

Lemma 3.12. There is a function $D \overline{3.12}: \mathbb{R}^{+} \rightarrow \mathbb{R}^{+}$such that the following holds. Suppose $I, J$ are intervals in $\mathbb{R}$ and $\bar{\phi}: I \rightarrow J$ is a $k$-quasi-isometric embedding. Let $x_{1}, x_{2}, x_{3} \in I, x_{1} \leq x_{2} \leq x_{3}$, and suppose $\phi\left(x_{1}\right)$ belongs to the interval with end points $\phi\left(x_{2}\right), \phi\left(x_{3}\right)$. Then $x_{2}-x_{1} \leq$ D.12 $(k)$.

Proof. Without loss of generality, we may assume that $\phi\left(x_{2}\right) \leq \phi\left(x_{1}\right) \leq \phi\left(x_{3}\right)$. Let $x_{4}=\inf \left\{y \in\left[x_{2}, x_{3}\right]: \phi(y) \geq \phi\left(x_{1}\right)\right\}$.

If $x_{2}=x_{4}$ then $\exists x^{\prime} \in\left[x_{2}, x_{2}+1\right] \cap\left[x_{2}, x_{3}\right]$ such that $\phi\left(x^{\prime}\right) \geq \phi\left(x_{1}\right)$. Now $x^{\prime}-x_{2} \leq 1$ implies $\left|\phi\left(x^{\prime}\right)-\phi\left(x_{2}\right)\right| \leq 2 k$, since $\phi$ is a $k$-quasi-isometric embedding. Therefore, $\phi\left(x_{1}\right)-\phi\left(x_{2}\right) \leq 2 k$. Thus we have $x_{2}-x_{1} \leq 3 k^{2}$.

If $x_{2}<x_{4}$ we choose $x^{\prime} \in\left[x_{2}, x_{4}\right)$ and $x^{\prime \prime} \in\left[x_{4}, x_{3}\right]$ such that $x_{4}-x^{\prime} \leq 1$ and $x^{\prime \prime}-x_{4} \leq 1$ with $\phi\left(x^{\prime \prime}\right) \geq \phi\left(x_{1}\right)$. Now $x^{\prime \prime}-x^{\prime} \leq 2$ implies $\left|\phi\left(x^{\prime}\right)-\phi\left(x^{\prime \prime}\right)\right| \leq 3 k$. Thus $\phi\left(x^{\prime \prime}\right)-\phi\left(x_{1}\right) \leq 3 k$, since $\phi\left(x^{\prime}\right)<\phi\left(x_{1}\right) \leq \bar{\phi}\left(x^{\prime \prime}\right)$ by the choices of $x^{\prime}, x^{\prime \prime}$. Hence $x_{2}-x_{1} \leq x^{\prime \prime}-x_{1} \leq 4 k^{2}$. Therefore, in any case, we may choose $D 3.12(k)=4 k^{2}$.

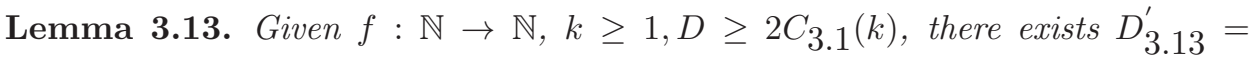
$D_{3.13}^{\prime}(f, k, D) \geq 1$ such that the following holds.

Suppose $p: X \rightarrow B$ is an $f$-metric graph bundle and $X_{1}, Y, X_{2}$ are $k$-qi sections in $X$. Also suppose that $Y$ is contained in the ladder $C\left(X_{1}, X_{2}\right)$. Then the $D$ neighborhood of each of the spaces $Y, C\left(X_{1}, Y\right), C\left(Y, X_{2}\right)$ is a connected subgraph of $X$ and the intersection of the spaces $C_{D}\left(X_{1}, Y\right)$ and $C_{D}\left(Y, X_{2}\right)$ is contained in the $D_{3.13}^{\prime}$ neighborhood of $Y$ in the path metric of both $C_{D}\left(X_{1}, Y\right)$ and $C_{D}\left(Y, X_{2}\right)$.

Proof. Since $X_{1}, X_{2}, Y$ are $k$-qi sections and $D \geq 2 C \overline{3.1}(k)$, it follows from Lemma 3.3 (1) that the $D$-neighborhood of each of the spaces $Y, C\left(X_{1}, Y\right), C\left(Y, X_{2}\right)$ is connected.

Now, let $y \in C_{D}\left(X_{1}, Y\right) \cap C_{D}\left(Y, X_{2}\right)$. Let us denote the path metric on $C_{D}\left(X_{i}, Y\right)$ induced from $X$ by $d_{i}$ and suppose $y_{i} \in C\left(X_{i}, Y\right)$ be such that $d_{i}\left(y, y_{i}\right) \leq D$, for $i=1,2$. Then $d_{B}\left(p\left(y_{1}\right), p\left(y_{2}\right)\right) \leq 2 D$. We need to prove the statements:

$\mathcal{P}_{j}$ : Any point of $C_{D}\left(X_{1}, Y\right) \cap C_{D}\left(Y, X_{2}\right)$ is contained in a $D^{\prime}$-neighborhood of $Y$ in $C_{D}\left(X_{j}, Y\right)$, for $j=1,2$.

Since the proofs of $\mathcal{P}_{1}, \mathcal{P}_{2}$ are similar, we shall only prove $\mathcal{P}_{2}$. We know that there exists a $k^{\prime}=G 3.1(k)$-qi section $Y_{2}$ say, through $y_{2} \in C\left(X_{2}, Y\right)$ contained in $C\left(X_{2}, Y\right)$. Join $y_{2}$ to the point $y_{1}^{\prime}=Y_{2} \cap F_{p\left(y_{1}\right)}$, by the lift of a geodesic in $B$ joining $p\left(y_{1}\right)$ and $p\left(y_{2}\right)$. The length of this path is at most $4 D k^{\prime}$ by Lemma 3.3 (2). Then $d\left(y_{1}, y_{1}^{\prime}\right) \leq 2 D+4 D k^{\prime}$ and hence their horizontal distance is at most $f\left(2 D+4 D k^{\prime}\right)$ by the bounded flaring condition for metric graph bundles. Thus choosing $D^{\prime}{ }_{3.13}$ to be $D+f\left(2 D+4 D k^{\prime}\right)$, we are through. 
Suppose $X_{1}, X_{2}$ are any two $c_{1}$-qi sections in $X$. Let us define the notation $c_{i+1}=C_{3.1}^{i}\left(c_{1}\right), i \geq 1$, as in the proof of Proposition 3.4. Then we have the following.

Proposition 3.14. For any $L \geq 2 c_{6}$, and $c_{1} \geq 1$ as above, there exists $\oint_{3.14}=$ [3.14 $\left(c_{1}, L\right)$ such that $C_{L}\left(X_{1}, X_{2}\right)$ is a $\overline{3.14}$ hyperbolic metric space with respect to the path metric induced from $X$.

Proof. Let $A=A_{2.22}^{\prime \prime}\left(c_{3}\right)+D{ }_{3.12}\left(2 g\left(c_{3}\right)\right)+f\left(2 L+4 c_{3} L\right)$. The idea of the proof is to break the ladder $C\left(X_{1}, X_{2}\right)$ into a finite number of subladders. Then by Proposition 3.4 and, if necessary, by a simple application of Corollary 1.52 we show that these subladders are hyperbolic. Finally we apply Corollary 1.52 again to the ladder assembled out of subladders to finish the proof.

Step 1 : Defining subladders.

Fix a horizontal geodesic $\mathcal{I}=F_{b_{0}} \cap C\left(X_{1}, X_{2}\right)$. The two end points of $\mathcal{I}$ lie in $X_{1}$ and $X_{2}$. Choose a parametrization $\alpha:[0, l] \rightarrow \mathcal{I}$ by arc length so that $\alpha(0) \in X_{1}$ and $\alpha(l) \in X_{2}$. We shall inductively construct a finite sequence of integers $0=s_{0}<$ $s_{1}<\cdots<s_{m}=l$, and a sequence of $c_{2}$-qi sections $X_{i}^{\prime}$ contained in $C\left(X_{1}, X_{2}\right)$ such that $X_{i}^{\prime}$ passes through $\alpha\left(s_{i}\right)$ for each $i=1, \cdots, m-1$. Let $X_{0}^{\prime}=X_{1}$. Suppose $s_{i}$ has been obtained, $s_{i}<l$ and $X_{i}^{\prime}$ has been constructed. If $d_{h}\left(X_{i}^{\prime}, X_{2}\right) \leq A$ then define $s_{i+1}=l, X_{i+1}^{\prime}=X_{2}$ and the construction is over. Otherwise, consider the set

$$
S_{i+1}=\left\{t \in\left[s_{i}, l\right] \cap \mathbb{N}: \exists \text { a } c_{2}-\text { qi section } X^{\prime} \text { through } \alpha(t) \text { with } d_{h}\left(X^{\prime}, X_{i}^{\prime}\right) \leq A\right\}
$$

Let $u_{i+1}=\max S_{i+1}$. If $\exists t \in S_{i+1}$ such that there is a $c_{2}$-qi section $X^{\prime}$ inside $C\left(X_{1}, X_{2}\right)$ through $\alpha(t)$ with $d_{h}\left(X^{\prime}, X_{i}^{\prime}\right)=A$, define $s_{i+1}=t$ and $X_{i+1}^{\prime}=X^{\prime}$. Otherwise define $s_{i+1}=\min \left\{l, u_{i+1}+1\right\}$ and let $X_{i+1}^{\prime}$ be any $c_{2}$-qi section inside $C\left(X_{1}, X_{2}\right)$ through $\alpha\left(s_{i+1}\right)$. The construction of these sections stops at the $m$-th step if $d_{h}\left(X_{m-1}^{\prime}, X_{2}\right) \leq A$, so that we must have $X_{m}^{\prime}=X_{2}$ and $s_{m}=l$. It follows from the above construction of the sections $X_{i}^{\prime}$ that for each $i, 1 \leq i \leq m-1$, we have $d_{h}\left(X_{i-1}^{\prime}, X_{i}^{\prime}\right) \geq A$ and in case $d_{h}\left(X_{i}^{\prime}, X_{i+1}^{\prime}\right)>A$, there is a section $X_{i}^{\prime \prime}$ through a point $\alpha\left(t_{i}\right), t_{i} \in\left[s_{i}, s_{i+1}\right]$ with $d_{h}\left(X_{j}^{\prime}, X_{i}^{\prime \prime}\right) \leq A, j=i, i+1$.

Step 2 : Subladders form a decomposition of $C\left(X_{1}, X_{2}\right)$.

In this step, we will show that $C\left(X_{1}, X_{2}\right)=\cup_{i=0}^{m-1} C\left(X_{i}^{\prime}, X_{i+1}^{\prime}\right)$ and that $C\left(X_{i-1}^{\prime}, X_{i}^{\prime}\right) \cap$ $C\left(X_{i}^{\prime}, X_{i+1}^{\prime}\right)=X_{i}^{\prime}$.

Note that the first assertion follows from the second and the construction in Step 1. For the second assertion, it is enough to show the following:

Claim: $X_{i+1}^{\prime} \subseteq C\left(X_{i}^{\prime}, X_{2}\right)$, for all $i, 1 \leq i \leq m-2$.

Consider the triples of points $\left(X_{1} \cap F_{b}, X_{i}^{\prime} \cap F_{b}, X_{i+1}^{\prime} \cap F_{b}\right), b \in \mathcal{V}(B)$. They are contained in the geodesic $F_{b} \cap C\left(X_{1}, X_{2}\right)$. For $b=b_{0}$ we know, by the construction in Step 1, that $X_{i}^{\prime} \cap F_{b} \in\left[X_{1} \cap F_{b}, X_{i+1}^{\prime} \cap F_{b}\right]$.

We now argue by contradiction. Suppose $X_{i+1}^{\prime} \nsubseteq C\left(X_{i}^{\prime}, X_{2}\right)$. Then for some point $b^{\prime} \in \mathcal{V}(B)$, we must have $X_{i+1}^{\prime} \cap F_{b^{\prime}} \in\left[X_{1} \cap F_{b^{\prime}}, X_{i}^{\prime} \cap F_{b^{\prime}}\right]$. Therefore there exist $b_{1}, b_{2} \in \mathcal{V}(B)$ with $d\left(b_{1}, b_{2}\right)=1$, such that $X_{i}^{\prime} \cap F_{b_{1}} \in\left[X_{1} \cap F_{b_{1}}, X_{i+1}^{\prime} \cap F_{b_{1}}\right]$ but $X_{i+1}^{\prime} \cap F_{b_{2}} \in\left[X_{1} \cap F_{b_{2}}, X_{i}^{\prime} \cap F_{b_{2}}\right]$. We know that $X_{i}^{\prime}, X_{i+1}^{\prime}$ are $c_{2}$-quasi-isometric sections, and $X_{1}$ is a $c_{1}$-quasi-isometric section. Hence $d\left(X_{i}^{\prime} \cap F_{b_{1}}, X_{i}^{\prime} \cap F_{b_{2}}\right) \leq 2 c_{2}$, $d\left(X_{i+1}^{\prime} \cap F_{b_{1}}, X_{i+1}^{\prime} \cap F_{b_{2}}\right) \leq 2 c_{2}$ and $d\left(X_{1} \cap F_{b_{1}}, X_{1} \cap F_{b_{2}}\right) \leq 2 c_{1} \leq 2 c_{2}$. 
By Lemma 3.1 the definition of $c_{3}$ (at the beginning of the proof of this proposition) and Lemma 1.13, we have a $g\left(2 c_{3}\right)$-quasi-isometric embedding $\left[X_{1} \cap F_{b_{1}}, X_{i+1}^{\prime} \cap\right.$ $\left.F_{b_{1}}\right] \rightarrow\left[X_{1} \cap F_{b_{2}}, X_{2} \cap F_{b_{2}}\right]$ which sends each of the points $X_{j}^{\prime} \cap F_{b_{1}}$ to $X_{j}^{\prime} \cap F_{b_{2}}$, $j=i, i+1$ and $X_{1} \cap F_{b_{1}}$ to $X_{1} \cap F_{b_{2}}$. By Lemma 3.12 we get

$$
d_{b_{1}}\left(X_{i}^{\prime} \cap F_{b_{1}}, X_{i+1}^{\prime} \cap F_{b_{1}}\right) \leq D \overline{3.12}\left(g\left(2 c_{3}\right)\right) .
$$

By the choice of the constant $A$, and the definition of $X_{i}^{\prime}$ 's this gives rise to a contradiction, completing the proof of Step 2.

Step 3: Subladders are uniformly hyperbolic. Next we show that there are constants $\delta_{1}, k_{1}$ and $D$ such that $(i)$ each $C_{L}\left(X_{i}^{\prime}, X_{i+1}^{\prime}\right)$ is $\delta_{1}$-hyperbolic and $X_{i}^{\prime}, X_{i+1}^{\prime}$ are $k_{1}$-quasi-convex in $C_{L}\left(X_{i}^{\prime}, X_{i+1}^{\prime}\right)$ for each $i, 0 \leq$ $i \leq m-1$. (ii) Also we shall show that the sets $X_{i}^{\prime}, X_{i+1}^{\prime}$ are mutually $D$-cobounded in $C_{L}\left(X_{i}^{\prime}, X_{i+1}^{\prime}\right)$, for $0 \leq i \leq m-1$.

(i) Since $X_{i}^{\prime}, X_{i+1}^{\prime}$ are $c_{2}$-qi sections in $X$, it follows that they are the images of $2 c_{2}$-quasi-isometric embeddings in $C_{L}\left(X_{i}^{\prime}, X_{i+1}^{\prime}\right)$ (Lemma 3.3(3)). Hence, they will be $D \overline{1.26}\left(\delta_{1}, 2 c_{2}\right)$-quasiconvex in $C_{L}\left(X_{i}^{\prime}, X_{i+1}^{\prime}\right)$ provided we can show that $C_{L}\left(X_{i}^{\prime}, X_{i+1}^{\prime}\right)$ is $\delta_{1}$-hyperbolic.

If $d_{h}\left(X_{i}^{\prime}, X_{i+1}^{\prime}\right) \leq A$ then, by Proposition 3.4, each $C_{L}\left(X_{i}^{\prime}, X_{i+1}^{\prime}\right)$ is $\overline{q_{3.4}}\left(c_{2}, A, L\right)$ hyperbolic; moreover, in this case, unless $i=m-1$, we have $d_{h}\left(X_{i}^{\prime}, X_{i+1}^{\prime}\right)=A$ and $X_{i}^{\prime}, X_{i+1}^{\prime}$ are then mutually $D \overline{3.4}\left(c_{2}, A, L\right)$-cobounded.

Suppose $d_{h}\left(X_{i}^{\prime}, X_{i+1}^{\prime}\right)>A$. Recall that $X_{j}^{\prime}$ passes through $\alpha\left(s_{j}\right), j=i, i+1$. In this case, we can find $t_{i} \in\left[s_{i}, s_{i+1}\right]$ such that there is a $c_{2}$-qi section $X_{i}^{\prime \prime}$ in $C\left(X_{1}, X_{2}\right)$, passing through $\alpha\left(t_{i}\right)$, so that $d_{h}\left(X_{j}^{\prime}, X_{i}^{\prime \prime}\right) \leq A, j=i, i+1$. Now, as in the proof of Lemma 3.1, we project points of $X_{i}^{\prime \prime}$ into the horizontal geodesics of $C\left(X_{i}^{\prime}, X_{i+1}^{\prime}\right)$ and get a $c_{3}$-qi section $Y_{i}^{\prime}$ through $\alpha\left(t_{i}\right)$. Note that we still have $d_{h}\left(X_{j}^{\prime}, Y_{i}^{\prime}\right) \leq A$ for $j=i, i+1$. By Proposition 3.4 $C_{L}\left(X_{i}^{\prime}, Y_{i}^{\prime}\right)$, and $C_{L}\left(X_{i+1}^{\prime}, Y_{i}^{\prime}\right)$ are both $\delta_{3.4}\left(c_{3}, A, L\right)$-hyperbolic. Also we see that $C_{L}\left(X_{i}^{\prime}, Y_{i}^{\prime}\right) \cap C_{L}\left(X_{i+1}^{\prime}, Y_{i}^{\prime}\right)$ contains a $2 c_{3}$-neighborhood of $Y_{i}^{\prime}$ which is connected. Since $Y_{i}^{\prime}$ is a $c_{3}$-quasiisometric image of $B$ in $X$, therefore it is a $2 c_{3}$-quasi-isometric image in both $C_{L}\left(X_{i}^{\prime}, Y_{i}^{\prime}\right)$ and $C_{L}\left(X_{i+1}^{\prime}, Y_{i}^{\prime}\right)$.

Now, we apply Lemma 3.13 followed by Corollary 1.52. Here the total space is $C_{L}\left(X_{i}^{\prime}, X_{i+1}^{\prime}\right)$ and we have just two subspaces: $C_{L}\left(X_{i}^{\prime}, Y_{i}^{\prime}\right)$ and $C_{L}\left(X_{i+1}^{\prime}, Y_{i}^{\prime}\right)$. Also their intersection contains a $2 c_{3}$-neighborhood of $Y_{i}^{\prime}$, denoted by $Y_{i}$, say. We see that the rest of the conditions of Corollary 1.52 are easily verified.

Thus, $C_{L}\left(X_{i}^{\prime}, X_{i+1}^{\prime}\right)$ is $\varepsilon_{1.52}\left(\Phi_{3.4}\left(c_{3}, A, L\right), D_{\underline{3.13}}^{\prime}\left(f, c_{3}, L\right), 1,2 c_{3}\right)$-hyperbolic. Choosing

$$
\delta_{1}:=\max \left\{\delta_{3.4}\left(c_{2}, A, L\right), \delta_{1.52}\left(\mathcal{Q}_{3.4}\left(c_{3}, A, L\right), D_{\overline{3.13}}^{\prime}\left(f, c_{3}, L\right), 1,2 c_{3}\right)\right\}
$$

completes the proof of Step 3(i).

(ii) We next show that the quasi-convex sets $X_{i}^{\prime}, X_{i+1}^{\prime}$ are mutually cobounded in $C_{L}\left(X_{i}^{\prime}, X_{i+1}^{\prime}\right)$.

Since the sets $U\left(X_{j}^{\prime}, Y_{i}^{\prime}\right), j=i, i+1$ are $K\left(=K\left[2.22\left(c_{3}, A\right)\right)\right.$-quasiconvex in $B$, the lift $Y_{i j}$ (say) of $U\left(X_{j}^{\prime}, Y_{i}^{\prime}\right)$ in $Y_{i}^{\prime}$ is a $C_{1}:=\left(2 K c_{3}+D_{1.26}\left(\delta_{1}, 2 c_{3}\right)\right)$-quasi-convex set in $C_{L}\left(X_{i}^{\prime}, X_{i+1}^{\prime}\right)$. 
Claim: There are constants $R=R\left(\delta_{1}, C_{1}\right), D_{1}=D_{1}\left(\delta_{1}, C_{1}\right)$ such that if $Y_{i j}$, $j=i, i+1$ are $R$-separated then the sets $X_{j}^{\prime}, j=i, i+1$ are $D_{1}$-cobounded.

Proof of Claim: We show that the projection of $X_{i+1}^{\prime}$ on $X_{i}^{\prime}$ is uniformly bounded. By a symmetric argument the projection of $X_{i}^{\prime}$ on $X_{i+1}^{\prime}$ is uniformly bounded.

Suppose $x \in X_{i+1}^{\prime}$ and let $y \in X_{i}^{\prime}$ be a nearest point projection of $x$ on $X_{i}^{\prime}$. Let $x_{1}$ be a nearest point projection of $x$ on $Y_{i}^{\prime}$ and let $y_{1}$ be a nearest point projection of $y$ on $Y_{i}^{\prime}$.

Sub-claim 1: The curve $\left[x, x_{1}\right] \cup\left[x_{1}, y_{1}\right] \cup\left[y_{1}, y\right]$ is a uniform quasi-geodesic if $R$ is sufficiently large.

Proof of Sub-claim 1: By Lemma1.31(2) the unions $\left[x, x_{1}\right] \cup\left[x_{1}, y_{1}\right]$ and $\left[x_{1}, y_{1}\right] \cup$ $\left[y_{1}, y\right]$ are $\left(3+2 C_{1}\right)$-quasi-geodesics. Sub-claim 1 will follow from the fact that $d\left(x_{1}, y_{1}\right) \geq L_{1.28}\left(\delta_{1}, 3+2 C_{1}, 3+2 C_{1}\right)$ for large enough $R$ (by Lemma 1.28).

By Lemma [1.36, if the sets $Y_{i j}$ are $R$-separated, $R \geq R_{1.36}\left(\delta_{1}, C_{1}\right)$ then there are points $y_{i j} \in Y_{i j}, j=i, i+1$ such that every geodesic connecting the sets $Y_{i j}$, $j=i, i+1$ passes through the $D \overline{1.36}\left(\delta_{1}, C_{1}\right)$-neighborhood of $y_{i j}, j=i, i+1$. Applying this to the geodesic $\left[x_{1}, y_{1}\right]$, Sub-claim 1 follows from the following.

Sub-claim 2: Suppose $x_{j}^{\prime} \in X_{j}^{\prime}$, and let $y_{j}^{\prime} \in Y_{i}^{\prime}$ be its nearest point projection on $Y_{i}^{\prime}$ for $j=i, i+1$. Then $y_{i j}$ is uniformly close to the geodesic $\left[x_{j}^{\prime}, y_{j}^{\prime}\right], j=i, i+1$.

Proof of Sub-claim 2: Since the proofs are similar, let us prove the statement for $j=i$. Let $b$ be a nearest point projection of $p\left(x_{i}^{\prime}\right)$ on $U\left(X_{i}^{\prime}, Y_{i}^{\prime}\right)$. Let $\alpha$ be a geodesic in $B$ joining $p\left(x_{i}^{\prime}\right)$ and $b$. Let $\beta$ be a geodesic joining $p\left(y_{i}^{\prime}\right)$ and $b$. Let $\tilde{\alpha}$ and $\tilde{\beta}$ be the lifts of $\alpha$ and $\beta$ in $X_{i}^{\prime}$ and $Y_{i}^{\prime}$ respectively. Let $\tilde{\alpha} \cap p^{-1}(b)=z_{i}$ and $\tilde{\beta} \cap p^{-1}(b)=w_{i}$. Then $d_{b}\left(z_{i}, w_{i}\right) \leq A$. The paths $\tilde{\alpha}$ and $\tilde{\beta}$ are $2 c_{1}$-quasi-geodesics in $C_{L}\left(X_{i}^{\prime}, X_{i+1}^{\prime}\right)$. Hence, by hyperbolicity of $C_{L}\left(X_{i}^{\prime}, X_{i+1}^{\prime}\right)$ there exist $x_{1}^{\prime \prime} \in\left[x_{i}^{\prime}, y_{i}^{\prime \prime}\right], x_{2}^{\prime \prime} \in \tilde{\alpha}, x_{3}^{\prime \prime} \in \tilde{\beta}$ which are uniformly close to each other (cf. Lemmas 1.26, 1.25). Then, it follows as in the first paragraph of the proof of Lemma 3.5 that $d_{p\left(x_{i}^{\prime}\right)}\left(X_{i}^{\prime}, Y_{i}^{\prime}\right)$ is uniformly bounded. Hence $y_{i i}$ is close to $x_{i}^{\prime}$ by Lemma 2.22 (1). Sub-claim 2 follows.

Since $C_{L}\left(X_{i}^{\prime}, X_{i+1}^{\prime}\right)$ is hyperbolic the Hausdorff distance between the quasigeodesic $\left[x, x_{1}\right] \cup\left[x_{1}, y_{1}\right] \cup\left[y_{1}, y\right]$ and the geodesic $[x, y]$ is uniformly bounded. Hence the points $y_{i i}$ and $y_{i i+1}$ are uniformly close to the geodesic $[x, y]$ by Sub-claim 2 . The Claim follows.

Finally, note that if $Y_{i j}, j=i, i+1$ are not $R$-separated then there exists a pair of points in $X_{i}^{\prime}$ and $X_{i+1}^{\prime}$ which are at a distance of at most $A_{1}^{\prime}:=(2 A+R)$ from each other. It follows as in the first paragraph of the proof of Lemma 3.5 that $d_{h}\left(X_{i}^{\prime}, X_{i+1}^{\prime}\right) \leq A_{1}:=f\left(2 A_{1}^{\prime} c_{2}+A_{1}^{\prime}\right)$. Hence, by Proposition 3.4 $X_{i}^{\prime}, X_{i+1}^{\prime}$ are $D \overline{3.4}\left(c_{2}, A_{1}, L\right)$-cobounded.

It follows that any geodesic joining $X_{j}^{\prime}, j=i, i+1$ passes close to the end points of this coarsely unique geodesic and step 3 follows.

Step 4 : The final step:

Finally we use Lemma 3.13 in conjunction with Corollary 1.52 Here the total space is $C_{L}\left(X_{1}, X_{2}\right)$, and the sequence of subspaces are $C_{L}\left(X_{i}^{\prime}, X_{i+1}^{\prime}\right), i=0,1, \ldots, m-1$. We check to see that the hypotheses of Corollary 1.52 are satisfied:

(1) Each of the subspaces $C_{L}\left(X_{i}^{\prime}, X_{i+1}^{\prime}\right)$ is $\delta_{1}$-hyperbolic by step 3;

(2) by choice of the constant $A>f\left(2 L+4 c_{3} L\right)$ (see Lemma 3.5) we know that only the consecutive ones intersect nontrivially;

(3) for $i=2, \ldots, m$, the intersection of two consecutive subspaces $C_{L}\left(X_{j}^{\prime}, X_{j+1}^{\prime}\right)$, 
$j=i-1, i$, contains the $2 c_{2}$-neighborhood $Y_{i}$ (say), of $X_{i}^{\prime}$. Also $Y_{i}$ is connected (Lemma 3.5). Further the intersection is contained in the $D_{3.13}^{\prime}\left(f, c_{3}, L\right)$ neighborhood of $Y_{i}$ in the spaces $C_{L}\left(X_{j}^{\prime}, X_{j+1}^{\prime}\right), j=i-1, i$;

(4) To check Condition (4) of Corollary 1.52 it is enough to show the following: Suppose $Z \subset X$ is a connected subgraph such that $Y_{i} \subset Z$. Then the inclusion $Y_{i} \hookrightarrow Z$ is uniform qi embedding.

The inclusion of $Y_{i}$ in the space $Z$ is clearly distance decreasing. Let $x, y \in Y_{i}$ and choose $x_{1}, y_{1} \in X_{i}^{\prime}$ such that $d\left(x, x_{1}\right) \leq 2 c_{2}, d\left(y, y_{1}\right) \leq 2 c_{2}$. Suppose $d_{Z}(x, y)=n$. Then $d_{X}\left(x_{1}, y_{1}\right) \leq d_{Z}\left(x_{1}, y_{1}\right) \leq n+4 c_{2}$. Hence $d_{B}\left(p\left(x_{1}\right), p\left(y_{1}\right)\right) \leq d_{X}\left(x_{1}, y_{1}\right) \leq$ $n+4 c_{2}$. Since $X_{i}^{\prime}$ is a $c_{2}$-qi section in $X$, by Lemma 3.3(2) there is a path of length $2 c_{2}\left(n+4 c_{2}\right)$ joining $x_{1}$ and $y_{1}$ contained in $Y_{i}$. Hence we have $d_{Y_{i}}(x, y) \leq$ $2 c_{2}\left(n+4 c_{2}\right)+4 c_{2}=2 c_{2} \cdot n+12 c_{2}$. This proves $(4)$.

(5) the sets $X_{i}^{\prime}, X_{i+1}^{\prime}$ are uniformly cobounded in $C_{L}\left(X_{i}^{\prime}, X_{i+1}^{\prime}\right)$ for $i=1,2, \ldots, m-$ 2 as proved in Step 3.

The proposition follows.

\section{The Combination Theorem}

As in Section 3, we assume the following for the purposes of this section:

1) $p: X \rightarrow B$ will be either an $f$ - metric graph bundle satisfying a flaring condition, or an approximating $(f-)$ metric graph bundle obtained from a metric bundle satisfying a flaring condition.

2) $B$ is $\delta$-hyperbolic and the horizontal spaces $F_{b}$ are $\delta^{\prime}$-hyperbolic for all vertices $b$ of $\mathcal{V}(B)$.

3) The barycenter maps $\partial^{3} F_{b} \rightarrow F_{b}$ are (uniformly) coarsely surjective. Thus by Proposition 2.10 we know that the metric graph bundle admits uniform $\left(K_{0}\right.$, say) qi sections through each point of $X$.

In this section we prove the main theorem of our paper which says that a metric (graph) bundle satisfying the above conditions has hyperbolic total space.

Here is an outline of the main steps of the proof:

For each pair of points $x, y \in X$, choose a ladder $C\left(X_{1}, X_{2}\right)$ containing $x, y$ and choose a geodesic $c(x, y)$ in $C_{D}\left(X_{1}, X_{2}\right)$ joining $x, y$ (with $D$ large enough but fixed). This gives a family of curves. We shall show that the family satisfies the conditions of Corollary 1.40 Proofs of conditions 1 and 2 follow from the results of the last section. Proofs of conditions 3 and 4 follow from Proposition 4.2 below, which contains the statement that large neighborhoods of 'tripod bundles' are hyperbolic. Proposition 4.2 in turn follows from Proposition 3.4 and Corollary 1.52

Definition 4.1. For three qi sections $X_{1}, X_{2}, X_{3}$ in a metric graph bundle $X$ over $B$ a tripod bundle determined by these qi sections, denoted $C\left(X_{1}, X_{2}, X_{3}\right)$, is defined to be the union of the ladders $C\left(X_{1}, X_{2}\right), C\left(X_{2}, X_{3}\right), C\left(X_{3}, X_{1}\right)$.

The convention that we adopted in Remark 2.14 applies here as well; namely, since the Hausdorff distance between any two tripod bundles determined by three qi sections is uniformly bounded (by hyperbolicity of the fibers), we denote by $C\left(X_{1}, X_{2}, X_{3}\right)$ any tripod bundle determined by the qi sections $X_{1}, X_{2}, X_{3}$. Also for any qi sections $X_{1}, X_{2}, X_{3}$ in $X$ and $D \geq 0$ we denote by $C_{D}\left(X_{1}, X_{2}, X_{3}\right)$ the $D$-neighborhood of the tripod bundle $C\left(X_{1}, X_{2}, X_{3}\right)$ in $X$.

The main technical tool of this section is the following: 
Proposition 4.2. Let $X$ over $B$ be an $(f, K)$-metric graph bundle such that i) $X$ is either a metric graph bundle satisfying a flaring condition or one obtained as an approximating metric graph bundle of a metric bundle satisfying a flaring condition:

ii) $B$ is $\delta$-hyperbolic and the horizontal spaces $F_{b}$ are $\delta^{\prime}$-hyperbolic for all vertices $b$ of $\mathcal{V}(B)$.

iii) the barycenter maps $\partial^{3} F_{b} \rightarrow F_{b}$ are (uniformly) coarsely surjective.

Given $c_{1} \geq 1$, there exists $L_{0}, \Phi_{4.5}, K_{4.5} \geq 0$ such that the following holds.

Let $X_{1}, X_{2}, X_{3}$ be $c_{1}$-qi sections and $L \geq L_{0}$. Then

(1) $C_{L}\left(X_{1}, X_{2}, X_{3}\right)$ is $q_{4.5}\left(=\Phi 4.5\left(c_{1}, L\right)\right)$-hyperbolic with the path metric induced from $X$ and each of $C_{L}\left(X_{i}, X_{j}\right), i \neq j$ is $K_{4.5}\left(=K_{4.5}\left(c_{1}, L\right)\right)$-quasiconvex in $C_{L}\left(X_{1}, X_{2}, X_{3}\right)$.

(2) there exists $D \overline{4.11}\left(=D 4.11\left(c_{1}, L\right)\right)$ such that if $x, y \in C_{L}\left(X_{1}, X_{2}\right), \gamma_{1}$ is a geodesic in $C_{L}\left(X_{1}, X_{2}, X_{3}\right)$ joining $x, y$ and $\gamma_{2}$ is a geodesic in $C_{L}\left(X_{1}, X_{2}\right)$ joining $x, y$, then the Hausdorff distance $H d\left(\gamma_{1}, \gamma_{2}\right) \leq D 4.11$.

(3) there exists $D \overline{4.12}\left(=D \overline{4.12}\left(c_{1}, L\right)\right)$ such that if $X_{i}, X_{i}^{\prime}, i=1,2$ are $c_{1}$-qi sections and $x_{i} \in X_{i} \cap X_{i}^{\prime}, i=1,2$, then the Hausdorff distance between the geodesics joining $x_{1}, x_{2}$ in the subspaces $C_{L}\left(X_{1}, X_{2}\right)$ and $C_{L}\left(X_{1}^{\prime}, X_{2}^{\prime}\right)$ is at most $D \overline{4.12}\left(c_{1}, L\right)$.

We postpone the proof of Proposition 4.2 to Section 4.1. Conclusions (1), (2), (3) above form the content of Proposition 4.5. Corollary 4.11 and Corollary 4.12 respectively. We give the proof of the main combination Theorem assuming Proposition 4.2 .

Theorem 4.3. Suppose $p: X \rightarrow B$ is a metric bundle (resp. metric graph bundle) such that

(1) $B$ is a $\delta$-hyperbolic metric space.

(2) Each of the fibers $F_{b}, b \in B$ (resp. $b \in \mathcal{V}(B)$ ) is a $\delta^{\prime}$-hyperbolic metric space with respect to the path metric induced from $X$.

(3) The barycenter maps $\partial^{3} F_{b} \rightarrow F_{b}, b \in B$ (resp. $b \in \mathcal{V}(B)$ ) are (uniformly) coarsely surjective.

(4) A flaring condition is satisfied.

Then $X$ is a hyperbolic metric space.

Proof. If $X$ is a metric bundle, we first replace $X$ by an approximating metric graph bundle. Abusing notation slightly, we continue to call the approximating metric graph bundle $X$. By Proposition 2.10, there exists $c_{1} \geq 1$ such that there is a $c_{1}$-qi section through each point of $\mathcal{V}(X)$.

Let $L=L_{0}$ be the constant given by Proposition 4.2 (1). We shall now define a set of curves joining pairs of points $x, y \in X$.

Definition of curve family: For each pair of points $x, y$ in $\mathcal{V}(X)$, choose, once and for all, two $c_{1}$-qi sections $X_{1}, X_{2}$ passing through $x$ and $y$ respectively. Now define $c(x, y)$ to be consecutive vertices on a geodesic in $C_{L}\left(X_{1}, X_{2}\right)$ joining $x, y$. We show that the family $\{c(x, y)\}$ satisfies properties (1)-(4) of Corollary 1.40 to complete the proof. As per the notation of Corollary 1.40, set $D=L$.

- Proof of property 1: This follows by taking $C_{1}=1$.

- Proof of property 2: By the first part of Lemma 3.5. Property 2 follows.

- Proof of property 3: This follows from Conclusions (1) and (2) of Proposition 4.2 
- Proof of property 4: Given $x, y, z \in X$ choose three $c_{1}$-qi sections $X_{3}, X_{4}, X_{5}$ containing $x, y, z \in X$ respectively and define the curves $c^{\prime}(x, y)$, $c^{\prime}(x, z)$ and $c^{\prime}(y, z)$ using these sections in the same way as the curves $c(x, y)$ are defined. It follows from Conclusion (2) of Proposition 4.2 that the triangle formed by $c^{\prime}(x, y), c^{\prime}(x, z)$ and $c^{\prime}(y, z)$ is $\left(\Phi_{4.5}\left(c_{1}, L\right)+2 D \overline{4.11}\left(c_{1}, L\right)\right)$ slim. Conclusion (3) of Proposition 4.2 now gives property 4.

Hyperbolicity of $X$ now follows from Corollary 1.40 .

Remark 4.4. Note that the conditions of Theorem 4.3 are inherited by induced metric graph bundles over quasi-isometrically embedded subsets of $B$. Hence the induced bundles over quasi-isometrically embedded subsets of $B$ are also hyperbolic.

4.1. Proof of Proposition 4.2. Suppose that $X_{1}, X_{2}$ and $X_{3}$ are three $c_{1}$-qi sections in $X$. The main aim of this subsection is to show that for large $D \geq$ $0, C_{D}\left(X_{1}, X_{2}, X_{3}\right)$ is hyperbolic. For this, we first show that taking a nearest point projection of $X_{3} \cap F_{b}$ onto the horizontal geodesic $C\left(X_{1}, X_{2}\right) \cap F_{b}$ (for all $b \in \mathcal{V}(B)$ ) we get a qi section $X_{4}$. (See figure below.) Then we have a genuine 'tripod bundle' $C\left(X_{1}, X_{2}\right) \cup C\left(X_{3}, X_{4}\right)$, such that $C_{D}\left(X_{1}, X_{2}, X_{3}\right)$ is quasiisometric to an $L$-neighborhood of $C\left(X_{1}, X_{2}\right) \cup C\left(X_{3}, X_{4}\right)$, where $L$ depends on $D$ and the bundle. The quasi-isometry is provided by projecting any point $z$ of $C_{L}\left(X_{1}, X_{2}\right) \cup C_{L}\left(X_{3}, X_{4}\right)$ onto a nearest point in $C_{D}\left(X_{1}, X_{2}, X_{3}\right)$ lying in the same horizontal fiber as $z$ (Here, the nearest point-projection is taken in the metric on the horizontal fiber to which $z$ belongs.) Hyperbolicity of the space $C_{L}\left(X_{1}, X_{2}\right) \cup C_{L}\left(X_{3}, X_{4}\right)$, and quasi-convexity of $C\left(X_{1}, X_{2}\right)$ in this space essentially follow from Proposition 3.14 and Corollary 1.52

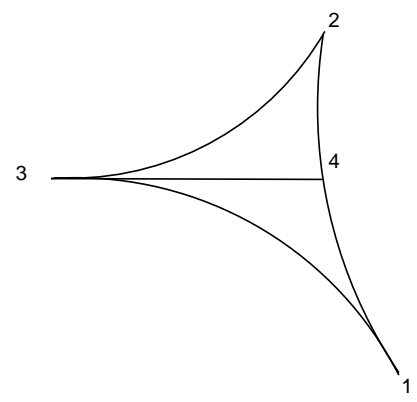

Tripod

Conclusion (1) of Proposition 4.2 is given by the following.

Proposition 4.5. Given $c_{1} \geq 1$, there exists $L_{0}, \Phi_{4.5}, K_{4.5} \geq 0$ such that the following holds.

Let $X_{1}, X_{2}, X_{3}$ be $c_{1}$-qi sections and $L \geq L_{0}$. Then $C_{L}\left(X_{1}, X_{2}, X_{3}\right)$ is $\overline{4.5}(=$ $\left.\Phi_{4.5}\left(c_{1}, L\right)\right)$-hyperbolic with the induced path metric from $X$ and each of $C_{L}\left(X_{i}, X_{j}\right)$, $i \neq j$ is $K_{[.5}\left(=K_{4.5}\left(c_{1}, L\right)\right)$-quasi-convex in $C_{L}\left(X_{1}, X_{2}, X_{3}\right)$.

For ease of exposition, we break the proof up into several lemmas, many of which will be minor modifications of results we have shown already.

For $b_{1}, b_{2} \in \mathcal{V}(B)$ with $d\left(b_{1}, b_{2}\right)=1$, we have a $g\left(2 c_{1}\right)$-quasi-isometry $F_{b_{1}} \rightarrow F_{b_{2}}$ by Lemma 1.13, which sends $X_{i} \cap F_{b_{1}}$ to $X_{i} \cap F_{b_{2}}$ for $i=1,2,3$. Therefore, by Lemma 1.38, choosing a nearest point projection of $X_{3} \cap F_{b}$ onto the horizontal geodesic $\left[X_{1} \cap F_{b}, X_{2} \cap F_{b}\right]$, for all $b \in B$, we get a $c_{1}^{\prime}$-qi section of $B$ in $X$ where 
$c_{1}^{\prime}:=2 c_{1}+D_{1.38}\left(\delta^{\prime}, g\left(2 c_{1}\right)\right)$. Let us call this section $X_{4}$. Let $c_{i+1}^{\prime}:=C_{[3.1}^{i}\left(c_{1}^{\prime}\right)$, $i \geq 1$.

Now we have the following analog of Lemma 3.13

Lemma 4.6. For all $L \geq 2 c_{2}^{\prime}$, there exists $D_{4.6}\left(=D_{4.6}(L)\right)$ such that the intersection $C_{L}\left(X_{1}, X_{2}\right) \cap C_{L}\left(X_{3}, X_{4}\right)$ is contained in the $\overline{4} 4.6$ - neighborhood of $X_{4}$.

Proof. The proof is an exact copy of that of the proof of Lemma 3.13, The only observation we need to make is that the curve $\left[X_{3} \cap F_{b}, X_{4} \cap F_{b}\right] \cup\left[X_{4} \cap F_{b}, X_{i} \cap F_{b}\right]$, $i=1,2$ is a $(3,0)$-quasi-geodesic in $F_{b}$ (Lemma 1.31(1)).

Lemma 4.7. For all $c_{1}$ as above and $L \geq 2 c_{6}^{\prime}$, there exist $D \overline{4.7}\left(=D \overline{4.7}\left(c_{1}, L\right)\right)$ and $K \overline{4.7}\left(=K K_{4.7}\left(c_{1}, L\right)\right)$ such that the space $C_{L}\left(X_{1}, X_{2}\right) \cup C_{L}\left(X_{3}, X_{4}\right)$ is $D 4.75$ hyperbolic and $C\left(X_{1}, X_{2}\right)$ is $K_{4.7}$ quasi-convex in this space.

Proof. The first part of the lemma follows as an application of Proposition 3.14 and Corollary 1.52 (the proof is a replica of Step 3 of the proof of Proposition 3.4 which shows that large girth subladders are hyperbolic). For completeness we briefly check the conditions of Corollary 1.52 ,

(1) Here we have only two subgraphs $C_{L}\left(X_{1}, X_{2}\right)$ and $C_{L}\left(X_{3}, X_{4}\right)$ which are hyperbolic by Proposition 3.4 .

(2) Condition (2) is trivially satisfied.

(3) The intersection $C_{L}\left(X_{1}, X_{2}\right) \cap C_{L}\left(X_{3}, X_{4}\right)$ contains the $c_{2}^{\prime}$-neighborhood, say $Y$, of $X_{4}$ which is connected and the rest follows from Lemma 4.6 above.

(4) Since $X_{4}$ is $2 c_{1}^{\prime}$-quasi-isometrically embedded in $C_{L}\left(X_{1}, X_{2}\right) \cup C_{L}\left(X_{3}, X_{4}\right)$, $Y=N_{2 c_{1}^{\prime}}\left(X_{4}\right)$ is also quasi-isometrically embedded.

(5) Condition 5 is trivially satisfied.

For the second part of the lemma we note that any geodesic joining two points of $C\left(X_{1}, X_{2}\right)$ in $C_{L}\left(X_{1}, X_{2}\right) \cup C_{L}\left(X_{3}, X_{4}\right)$ and which leaves $C_{L}\left(X_{1}, X_{2}\right)$ must join two points in a (uniformly bounded) neighborhood of $X_{4}$, by Lemma 4.6. Since $X_{4}$ is the image of a quasi-isometric embedding of $B$ in the hyperbolic space $C_{L}\left(X_{1}, X_{2}\right) \cup$ $C_{L}\left(X_{3}, X_{4}\right)$ it is quasi-convex also. The lemma follows.

Clearly for all $b \in \mathcal{V}(B), C\left(X_{1}, X_{2}, X_{3}\right) \cap F_{b}$ is $\delta^{\prime}$-quasi-convex in $F_{b}$. Define a map $\Pi: Z=C_{L}\left(X_{1}, X_{2}\right) \cup C_{L}\left(X_{3}, X_{4}\right) \rightarrow X$ by sending any point $x \in Z \cap F_{b}$ to a nearest point in $C\left(X_{1}, X_{2}, X_{3}\right) \cap F_{b}$ (in the $d_{b}$-metric).

Lemma 4.8. Given $c_{1} \geq 1$ there exists $D \overline{4.8}\left(=D \overline{4.8}\left(c_{1}\right)\right)$ such that the map $\Pi$ is D4.8-coarsely Lipschitz.

Proof. We need to check that for any two adjacent vertices in the domain of $\Pi$, the image vertices are at a uniformly bounded distance. This breaks up into two cases.

When the vertices are in the same horizontal space $F_{b}$ then since $C\left(X_{1}, X_{2}, X_{3}\right) \cap$ $F_{b}$ is (uniformly) quasiconvex in $F_{b}$, and since nearest point projections onto quasiconvex sets in hyperbolic metric spaces are coarsely Lipschitz (cf. Lemma 3.2 of Mit98b) the claim follows.

When the vertices are not in same horizontal space then the same argument as in Lemma 1.38 (also see Mit98b, Bow07) shows that nearest-point projections and quasi-isometries almost commute. The rest of the proof is a replica of Theorem 3.2 Mit98a. 
Remark 4.9. In fact, $\Pi$ restricted to $C\left(X_{1}, X_{2}\right)$ is simply an inclusion map. Hence by Lemma 3.5. $\Pi$ is a qi-embedding of $C\left(X_{1}, X_{2}\right)$ into any sufficiently large neighborhood of $C\left(X_{1}, X_{2}, X_{3}\right)$ equipped with a path metric induced from $X$.

Lemma 4.10. Given $c_{1} \geq 1$ and $L \geq 0$ as above there exists $D\left[4.10\left(=D \overline{4.10}\left(c_{1}, L\right)\right)\right.$ such that the following holds.

For all $x \in C_{L}\left(X_{1}, X_{2}\right) \cup C_{L}\left(X_{3}, X_{4}\right)$ the horizontal distance between $x$ and $\Pi(x)$ is at most $D 4.10$.

Proof. This follows from the fact that in any $\delta^{\prime}-$ hyperbolic metric space $\left(=F_{b}\right.$ in our case) the Hausdorff distance between a triangle with vertices $x, y, z$ and the tripod $[x, w] \cup[y, z]$ (where $w \in[y, z]$ is a nearest point projection of $x$ onto $[y, z]$ ) is bounded by $\delta^{\prime}$.

\section{Proof of Proposition 4.5;}

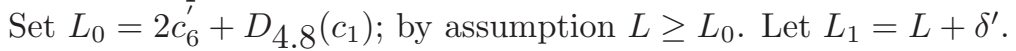

First for every pair of points in $x, y \in C\left(X_{1}, X_{2}, X_{3}\right)$ we choose a geodesic in (the path metric induced on) $C_{L_{1}}\left(X_{1}, X_{2}\right) \cup C_{L_{1}}\left(X_{3}, X_{4}\right)$ joining $x, y$ and project it into $C\left(X_{1}, X_{2}, X_{3}\right)$ by $\Pi$. This defines a path in $C\left(X_{1}, X_{2}, X_{3}\right)$ say $c(x, y)$ joining $x, y$. Note that by Lemma 4.10 the paths $c(x, y)$ are uniform quasi-geodesics in $C_{L_{1}}\left(X_{1}, X_{2}\right) \cup C_{L_{1}}\left(X_{3}, X_{4}\right)$. Now we need to check the conditions of Corollary 1.40.

Here the whole space is $C_{L}\left(X_{1}, X_{2}, X_{3}\right)$ and the discrete set is the set of vertices contained in $C\left(X_{1}, X_{2}, X_{3}\right)$. As per the notation of Corollary 1.40, set $D=L$. Next we note the following:

(1) Condition (1) of Corollary 1.40 follows from Lemma 4.8

(2) Condition (2) of Corollary 1.40 follows from the observation that $C_{L}\left(X_{1}, X_{2}, X_{3}\right)$ is contained in $C_{L_{1}}\left(X_{1}, X_{2}\right) \cup C_{L_{1}}\left(X_{3}, X_{4}\right)$.

(3) Conditions (3), (4) of Corollary 1.40 follow from Lemma 4.10, since the space $C_{L_{1}}\left(X_{1}, X_{2}\right) \cup C_{L_{1}}\left(X_{3}, X_{4}\right)$ is (uniformly) hyperbolic.

Hence $C_{L}\left(X_{1}, X_{2}, X_{3}\right)$ is hyperbolic. From Lemmas 4.7, and 4.10 it follows that $C\left(X_{1}, X_{2}\right)$ is the image of the quasi-convex set $C\left(X_{1}, X_{2}\right) \subset C_{L_{1}}\left(X_{1}, X_{2}\right) \cup$ $C_{L_{1}}\left(X_{3}, X_{4}\right)$ under the quasi-isometric embedding $\Pi$ (cf. Remark 4.9). Hence it is quasi-convex in $C_{L}\left(X_{1}, X_{2}, X_{3}\right)$ and thus so is $C_{L}\left(X_{1}, X_{2}\right)$. This completes the proof.

Conclusion (2) of Proposition 4.2 is given by the next Corollary, which is an immediate consequence of the fact that $C_{L}\left(X_{1}, X_{2}, X_{3}\right)$ is hyperbolic (cf. Proposition 4.5) and that the inclusion $C_{L}\left(X_{1}, X_{2}\right) \hookrightarrow C_{L}\left(X_{1}, X_{2}, X_{3}\right)$ is a qi embedding (cf. Remark 4.9).

Corollary 4.11. Given $c_{1} \geq 1$ and $L \geq L_{0}$ (where $L_{0}$ is as in Proposition 4.5) there exists $D_{4.11}\left(=D_{4.11}\left(c_{1}, L\right)\right)$ such that if $x, y \in C_{L}\left(X_{1}, X_{2}\right), \gamma_{1}$ is a geodesic in $C_{L}\left(X_{1}, X_{2}, X_{3}\right)$ joining $x, y$ and $\gamma_{2}$ is a geodesic in $C_{L}\left(X_{1}, X_{2}\right)$ joining $x, y$, then the Hausdorff distance $H d\left(\gamma_{1}, \gamma_{2}\right) \leq Q 4.11$.

Conclusion (3) of Proposition 4.2 is given by the following.

Corollary 4.12. Given $c_{1} \geq 1$ and $L \geq L_{0}$ (cf. Proposition 4.5) there exists $D_{4.12}\left(=D_{4.12}\left(c_{1}, L\right)\right)$ such that the following holds.

Suppose $X_{i}, X_{i}^{\prime}, i=1,2$ are $c_{1}$-qi sections and $x_{i} \in X_{i} \cap X_{i}^{\prime}, i=1,2$. Then the 
Hausdorff distance between the geodesics joining $x_{1}, x_{2}$ in the subspaces $C_{L}\left(X_{1}, X_{2}\right)$ and $C_{L}\left(X_{1}^{\prime}, X_{2}^{\prime}\right)$ is at most $D_{4.12}\left(c_{1}, L\right)$.

Proof. This follows from Proposition 4.5 and Corollary 4.11 applied successively to the tripod bundles $C_{L}\left(X_{1}, X_{1}^{\prime}, X_{2}\right)$ and $C_{L}\left(X_{1}^{\prime}, X_{2}, X_{2}^{\prime}\right)$.

Concluding the proof of Proposition 4.2; Proposition 4.5, Corollary 4.11 and Corollary 4.12 together give precisely the statement of Proposition 4.2 .

\section{Consequences and Applications}

A number of consequences of Theorem 4.3 are collected together in this section.

5.1. Sections, Retracts and Cannon-Thurston maps. We shall say that an exact sequence of finitely generated groups $1 \rightarrow K \rightarrow G \rightarrow Q \rightarrow 1$ satisfies bounded flaring if the associated metric graph bundle (cf. Example 1.8) of Cayley graphs does. An immediate consequence of Theorem 4.3 coupled with the existence of qi-sections from Theorem 2.11 is the following converse to (the second part of) Mosher's Theorem 2.11.

Theorem 5.1. Suppose that the short exact sequence of finitely generated groups

$$
1 \rightarrow K \rightarrow G \rightarrow Q \rightarrow 1 \text {. }
$$

satisfies a flaring condition such that $K, Q$ are word hyperbolic and $K$ is nonelementary. Then $G$ is hyperbolic.

Theorem 2.11 was generalized by Pal Pal10 as follows.

Theorem 5.2. (Pal Pal10]) Suppose we have a short exact sequence of pairs of finitely generated groups

$$
1 \rightarrow\left(K, K_{1}\right) \rightarrow\left(G, N_{G}\left(K_{1}\right)\right) \stackrel{p}{\rightarrow}\left(Q, Q_{1}\right) \rightarrow 1
$$

with $K$ strongly hyperbolic relative to a subgroup $K_{1}$ such that $G$ preserves cusps, i.e. for all $g \in G$ there exists $h \in K$ with $g K_{1} g^{-1}=h K_{1} h^{-1}$. Then there exists a $(k, \epsilon)$-quasi-isometric section $s: Q \rightarrow G$ for some constants $k \geq 1, \epsilon \geq 0$. Further, $Q_{1}=Q$ and there is a quasi-isometric section $s: Q \rightarrow N_{G}\left(K_{1}\right)$ satisfying

$$
\frac{1}{R} d_{Q}\left(q, q^{\prime}\right)-\epsilon \leq d_{N_{G}\left(K_{1}\right)}\left(s(q), s\left(q^{\prime}\right)\right) \leq R d_{Q}\left(q, q^{\prime}\right)+\epsilon
$$

where $q, q^{\prime} \in Q$ and $R \geq 1, \epsilon \geq 0$ are constants. In addition, if $G$ is weakly hyperbolic relative to $K_{1}$, then $Q$ is hyperbolic.

The setup of Theorem 5.2 naturally gives a metric graph bundle $P: X \rightarrow Q$ of spaces, where $Q$ is the quotient group and fibers are isometric to the coned off spaces $\widehat{K}$ obtained by electrocuting copies of $K_{1}$ in $K$.

We shall now use Theorem 3.2. Theorem 3.2 is proven in Mit98a in the context of an exact sequence of finitely generated groups $1 \rightarrow N \rightarrow G \rightarrow Q \rightarrow 1$, with $N$ hyperbolic; but all that the proof requires is the existence of qi sections (which follows in the context of groups by the qi section Theorem 2.11 of Mosher).

As in Mit98a, the existence of a qi-section through each point of $X$ guarantees, via Theorem 3.2, the existence of a continuous extension to the boundary (also called a Cannon-Thurston map [CT07. CT85) of the map $i_{b}: F_{b} \rightarrow X$ provided $X$ is hyperbolic. The proof is identical to that in Mit98a and we omit it here, referring the reader to Mit98a for details. Combining this fact with Theorem 4.3 we have the following. 
Theorem 5.3. Suppose $p: X \rightarrow B$ is a metric (graph) bundle with the following properties:

(1) $B$ is a $\delta$-hyperbolic metric space.

(2) Each of the fibers $F_{b}, b \in B(b \in \mathcal{V}(B))$ is a $\delta^{\prime}$-hyperbolic metric space with respect to the induced path metric from $X$.

(3) The barycenter maps $\partial^{3} F_{b} \rightarrow F_{b}, b \in B(b \in \mathcal{V}(B))$ are uniformly coarsely surjective.

(4) The metric (graph) bundle satisfies a flaring condition.

Then the inclusion $i_{b}: F_{b} \rightarrow X$ extends continuously to a map $\hat{i}: \widehat{F_{b}} \rightarrow \widehat{X}$ between the Gromov compactifications.

5.2. Hyperbolicity of base and flaring. In our main combination theorem 4.3 flaring was a sufficient condition. In this subsection and the next we investigate its necessity. This issue is closely linked with hyperbolicity of the base space $B$. We study it with special attention to hyperbolic and relatively hyperbolic groups as in Theorems 2.11 and Theorem 5.2

A Theorem of Papasoglu (cf. Pap95, Lemma 3.8 of [Pap05]) states the following.

Theorem 5.4. Pap95, Pap05 Let $G$ be a finitely generated group and let $\Gamma$ be the Cayley graph of $G$ with respect to a finite generating set. If there is an $\epsilon$ such that geodesic bigons in $\Gamma$ are $\epsilon$-thin then $G$ is hyperbolic.

Similarly, let $X$ be a geodesic metric space such that for every $K$ there exists $C$ such that $K$-quasigeodesic bigons are $C$-thin, then $X$ is hyperbolic.

In fact there is some (universal) constant $C>0$ such that if $G$ is finitely generated and non-hyperbolic, then $\forall R>0$ there is some $R^{\prime}>R$ and a $(C, C)$-quasi-isometric embedding of a Euclidean circle of radius $R^{\prime}$ in $\Gamma$.

We now look at short exact sequences of finitely generated groups.

Proposition 5.5. Consider a short exact sequence of finitely generated groups

$$
1 \rightarrow K \rightarrow G \rightarrow Q \rightarrow 1 \text {. }
$$

such that $K$ is non-elementary word hyperbolic but $Q$ is not hyperbolic. Then the short exact sequence cannot satisfy a flaring condition.

Proof. By Theorem 5.4 $Q$ contains $(C, C)$ qi embeddings of Euclidean circles of arbitrarily large radius. Now, given any $l, A_{0}$ construct

a) a $(C, C)$ qi embedding $\tau_{l}$ of a Euclidean circle $\sigma$ of circumference $>4 l$ in $Q$

b) two qi sections $s_{1}, s_{2}$ of $Q$ into $G$ by Theorem 2.11 such that $d_{h}\left(s_{1} \circ \tau_{l}(\sigma), s_{2} \circ\right.$ $\left.\tau_{l}(\sigma)\right)>A_{0}$.

Let $q \in \sigma$ be such that the horizontal distance $d_{q}\left(s_{1} \circ \tau_{l}(q), s_{2} \circ \tau_{l}(q)\right)$ in the fiber $F_{q}$ over $q$ is maximal. Let the two arcs of length $l$ in $\tau_{l}$ starting at $q$ (in opposite directions) end at $q_{1}, q_{2}$. Let $\overline{q_{1} q q_{2}}$ denote the union of these arcs. Then the two quasigeodesics $s_{1} \circ \tau_{l}\left(\overline{q_{1} q q_{2}}\right), s_{2} \circ \tau_{l}\left(\overline{q_{1} q q_{2}}\right)$ violate flaring as the horizontal distance achieves a maximum at the midpoint $q$.

We next turn to the relatively hyperbolic situation described in Theorem 5.2 with $Q$ non-hyperbolic, i.e. we assume that $K$ is (strongly) hyperbolic relative to $K_{1}$. We have an analog of Proposition [5.5 in this situation too. The proof is the same as that of the above proposition. The existence of qi sections in this case, follows from Theorem 5.2 
Lemma 5.6. Suppose we have a short exact sequence of finitely generated groups

$$
1 \rightarrow\left(K, K_{1}\right) \rightarrow\left(G, N_{G}\left(K_{1}\right)\right) \stackrel{p}{\rightarrow}\left(Q, Q_{1}\right) \rightarrow 1
$$

such that $K$ strongly hyperbolic relative to the cusp subgroup $K_{1}$ and $G$ preserves cusps, but $Q$ is not hyperbolic. Let $P: X \rightarrow Q$ be the associated metric graph bundle of spaces, where $Q$ is the quotient group and fibers $F_{q}$ are the coned off spaces $\widehat{K}$ obtained by electrocuting copies of $K_{1}$ in $K$. Then $X$ does not satisfy flaring.

The rest of this subsection is devoted to proving the following.

Proposition 5.7. Suppose we have a short exact sequence of finitely generated groups

$$
1 \rightarrow\left(K, K_{1}\right) \rightarrow\left(G, N_{G}\left(K_{1}\right)\right) \stackrel{p}{\rightarrow}\left(Q, Q_{1}\right) \rightarrow 1
$$

with $K$ (strongly) hyperbolic relative to the cusp subgroup $K_{1}$ such that $G$ preserves cusps. Suppose further that $G$ is (strongly) hyperbolic relative to $N_{G}\left(K_{1}\right)$. Then $Q$ is hyperbolic.

Proof. We shall argue by contradiction. Suppose $Q$ is not hyperbolic.

Let $X$ be a Cayley graph of $G$ with respect to a finite generating set $S$ containing a finite generating set of $K$ (and, for good measure, a finite generating set of $K_{1}$ ). Let $B$ be the Cayley graph of $Q$ with respect to $p(S) \backslash\{1\}$. Then the quotient map $G \rightarrow Q$ gives rise to a metric graph bundle $p: X \rightarrow B$ as before. This metric graph bundle admits uniform qi sections through each point of $X$ by Theorem 5.2. Also $B$ is not a hyperbolic metric space. By Theorem [5.4 there exists $C>0$ such that for all $r>0$ we can construct a $(C, C)$-qi embedding $\tau_{r}$ of a Euclidean circle $\sigma_{r}$ of radius bigger than $r$ in $B$.

Claim: Given $k>0$ there exists $D=D(k)$ such that for any $k$-qi section $s$ : $\mathcal{V}(B) \rightarrow X$ of the metric graph bundle $p: X \rightarrow B, s \circ \tau_{r}\left(\sigma_{r}\right)$ is contained in a $D$-neighborhood of a coset of $N_{G}\left(K_{1}\right)$.

Proof of claim: Let $\tau=s \circ \tau_{r}$. Then $\tau$ is a $k_{1}:=(k C+k)$-quasi-isometric embedding of $\sigma_{r}$ in $s(B)$. Let $u, v$ be a pair of antipodal points of the circle and $a=\tau(u), b=\tau(v)$. Let $\sigma_{r}^{1}, \sigma_{r}^{2}$ be the two arcs of $\sigma_{r}$ joining $u, v$. Then $\tau\left(\sigma_{r}^{1}\right), \tau\left(\sigma_{r}^{2}\right)$ are $k_{1}$-quasigeodesics joining $a, b$.

Let $d_{r}$ denote the intrinsic path metric on $\sigma_{r}$. Since $\tau$ is a qi embedding, it follows that for all $C_{1} \geq 0$, there exists $C_{2} \geq 0$ such that for all $r>0$ and $x \in \sigma_{r}^{1}, y \in \sigma_{r}^{2}, d_{r}(x,\{a, b\}) \geq C_{2}$ and $d_{r}(y,\{a, b\}) \geq C_{2}$ implies that $d_{X}(x, y) \geq C_{1}$. Hence $\tau\left(\sigma_{r}^{1}\right) \cup \tau\left(\sigma_{r}^{2}\right)$ is a 'thick' quasigeodesic bigon, i.e. except for initial and final subsegments of length $k_{1} C_{2}, \tau\left(\sigma_{r}^{1}\right)$ and $\tau\left(\sigma_{r}^{2}\right)$ are separated from each other by at least $\frac{C_{1}}{k_{1}}$.

Since $G$ is strongly hyperbolic relative to $N_{G}\left(K_{1}\right)$, thick quasigeodesic bigons lie in a bounded neighborhood of a coset of $N_{G}\left(K_{1}\right)$ (see Definition 1.41 or [Far98]). The claim follows.

We continue with the proof of the proposition. For any $k$-qi section $s: \mathcal{V}(B) \rightarrow$ $X$, we shall refer to $s \circ \tau_{r}\left(\sigma_{r}\right)=\tau\left(\sigma_{r}\right)$ as a qi section of the circle $\sigma_{r}$. Let $Y_{1}, Y_{2}$ be two $k$-qi sections of a large Euclidean circle $\sigma_{r}$ in $B$, such that $Y_{1}$ and $Y_{2}$ lie $D-$ close to two distinct cosets of $N_{G}\left(K_{1}\right)$ (with $D=D(k)$ as in the Claim above). Let $W\left(Y_{1}, Y_{2}\right)$ be the union $\cup_{q \in \tau_{r}\left(\sigma_{r}\right)} \lambda_{q}$, where $\lambda_{q}$ is a horizontal geodesic in $F_{q}$ joining $Y_{1} \cap F_{q}$ to $Y_{2} \cap F_{q}$. Suppose $b, b^{\prime}$ are images (under $\tau_{r}$ ) of antipodal points on $\sigma_{r}$. As in the proof of Lemma 3.1 we know that there exists $k_{1}\left(=k_{1}(k)\right)$ such that for 
each point $z$ of $\lambda_{b}$ there exists a $k_{1}$-quasi-isometric section of $\sigma_{r}$ in $W\left(Y_{1}, Y_{2}\right)$; any such qi section is $D_{1}\left(=D_{1}\left(k_{1}\right)\right)$-close to a coset of $N_{G}\left(K_{1}\right)$ by the Claim above.

Since $Y_{1}, Y_{2}$ are close to distinct cosets of $N_{G}\left(K_{1}\right)$, we can find (as in Step 1 of Proposition 3.14) two $k_{1}$-qi sections $Y_{1}^{\prime}, Y_{2}^{\prime}$ of $\sigma_{r}$ passing through $z_{1}, z_{2} \in \lambda_{b}$ with $d\left(z_{1}, z_{2}\right)=1$ such that $Y_{1}^{\prime}, Y_{2}^{\prime}$ are

a) $D_{1}$-close to two distinct cosets of $N_{G}\left(K_{1}\right)$,

b) both contained in $W\left(Y_{1}, Y_{2}\right)$.

Suppose $Y_{1}^{\prime}, Y_{2}^{\prime}$ intersect $\lambda_{b^{\prime}}$ in $z_{1}^{\prime}$ and $z_{2}^{\prime}$ respectively. If $d\left(z_{1}^{\prime}, z_{2}^{\prime}\right)$ is large, in the same way as before, we can construct two $k_{2}\left(=k_{2}\left(k_{1}\right)\right)$-qi sections $Y_{3}, Y_{4}$ of $\sigma_{r}$ contained in $W\left(Y_{1}^{\prime}, Y_{2}^{\prime}\right)$ such that

a) $Y_{3}, Y_{4}$ are $D_{2}\left(=D_{2}\left(D_{1}\right)\right)$ - close to two distinct cosets of $N_{G}\left(K_{1}\right)$

b) $d\left(Y_{3} \cap \lambda_{b^{\prime}}, Y_{4} \cap \lambda_{b^{\prime}}\right)=1$.

Thus we have two $k_{2}$-qi sections of long subarcs of $\sigma_{r}$ that start and end close by in $X$ but lie close to distinct cosets of $N_{G}\left(K_{1}\right)$. Since $r$ can be chosen to be arbitrarily large, this violates strong relative hyperbolicity of $G$ with respect to the cosets of $N_{G}\left(K_{1}\right)$, proving the proposition.

5.3. Necessity of Flaring. In this subsection we prove that flaring is a necessary condition for hyperbolicity of a metric (graph) bundle:

Proposition 5.8. Let $P: X \rightarrow B$ be a metric (graph) bundle such that 1. $X$ is $\delta$-hyperbolic

2. There exist $\delta_{0}$ such that each of the fibers $F_{z}, z \in B(\mathcal{V}(B))$ is $\delta_{0}$-hyperbolic equipped with the path metric induced from $X$.

Then the metric bundle satisfies a flaring condition. In particular, any exact sequence of finitely generated groups $1 \rightarrow N \rightarrow G \rightarrow Q \rightarrow 1$ with $N, G$ hyperbolic satisfies a flaring condition.

The proof will occupy the entire subsection. Suppose $\gamma:[-L, L] \rightarrow B$ is a geodesic and $\alpha, \beta$ are two $K_{1}$-qi lifts of $\gamma$. As in the construction of ladders, we define $Y$ to be the union of horizontal geodesics $[\alpha(t), \beta(t)] \subset F_{\gamma(t)}, t \in[-L, L]$, and refer to it as the ladder formed by $\alpha$ and $\beta$. Let $\eta:[0, M] \rightarrow F_{\gamma(0)}$ be the geodesic $Y \cap F_{\gamma(0)}$.

A crucial ingredient is the following lemma which is a specialization to our context of the fact that geodesics in a hyperbolic space diverge exponentially. (See Proposition 2.4 and the proof of Theorem 4.11 in [Mit97]).

Lemma 5.9. Given $K_{1} \geq 1, D \geq 0$ there exist $b=b\left(K_{1}, D\right)>1, A=A\left(K_{1}, D\right)>$ 0 and $C=C\left(K_{1}, D\right)>0$ such that the following holds:

If $d(\alpha(0), \beta(0)) \leq D$ and there exists $T \in[0, L]$ with $d(\alpha(T), \beta(T)) \geq C$ then any path joining $\alpha(T+t)$ to $\beta(T+t)$ and lying outside the union of the $\frac{T+t-1}{2 K_{1}}$-balls around $\alpha(0), \beta(0)$ has length greater than $A b^{t}$ for all $t \geq 0$ such that $T+t \in[0, L]$. In particular, the horizontal distance between $\alpha(T+t)$ and $\beta(T+t)$ is greater than $A b^{t}$ for all $t \geq 0$ such that $T+t \in[0, L]$.

Now, we use Lemma 5.9 to show that the ladder $Y$ flares in at least one direction of $\gamma$. We start the proof by showing this in two special cases. A general ladder is then broken into subladders of the special types by qi lifts of $\gamma$ as in Step 1 of the proof of Proposition 3.14. (Recall that we get exactly two types of subladders in this way. This motivates us to consider the two types of special ladders here.) We point out that 
(1) the first type of ladder is of uniformly small (but not too small) girth;

(2) the second type of ladder is not necessarily of small girth but any qi lift of $\gamma$ divides it into two subladders of small girth.

The proof of flaring for all ladders follows from this.

We shall need the following lemma also.

Lemma 5.10. 1) Given $d_{1}, d_{2}, \delta \geq 0$ and $k \geq 1$ there are constants $C=C\left(d_{1}, d_{2}, k, \delta\right)$ and $D=D(k, \delta)$ such that the following holds:

Let $X$ be a $\delta$-hyperbolic metric space and let $\alpha_{1}, \alpha_{2}:[-L, L] \rightarrow X$ be $k$-quasigeodesics. Let $[a, b] \subset[-L, L]$ and suppose $d_{1}=d\left(\alpha_{1}(a), \alpha_{2}(a)\right)$ and $d_{2}=d\left(\alpha_{1}(b), \alpha_{2}(b)\right)$. If $[t-C, t+C] \subset[a, b]$ for some $t \in[a, b]$ then $d\left(\alpha_{1}(t), \alpha_{2}(t)\right) \leq D$.

2) Through each point of a ladder $Y$ formed by $K_{1}$-qi lifts of a geodesic $\gamma$ in $B$ there is a $C_{3.1}\left(K_{1}\right)$-qi lift of $\gamma$ contained in $Y$.

Proof. (1) follows easily from stability of quasi-geodesics and slimness of triangles in $X$. (See Lemma 1.15 of Chapter III.H, BH99] for instance).

(2) This is a replica of the proof of Lemma 3.1

Remark 5.11. We shall assume $L$ to be sufficiently large for the following arguments to go through. We give the proof for metric bundles. The same proof works mutatis mutandis (replacing $B$ by $(\mathcal{V}(B)$ ) for instance) for metric graph bundles.

Flaring of ladders in special cases:

Let $D=D_{5.10}\left(K_{1}, \delta\right)$ and $D_{1}^{\prime}=C_{5.9}\left(K_{1}, D\right)$. Since the horizontal spaces in $X$ are uniformly properly embedded in $\bar{X}$ there is a $D_{1}$ such that for all $v \in B$ and $x, y \in F_{v}$ if $d_{v}(x, y) \geq D_{1}$ then $d(x, y) \geq D_{1}^{\prime}$. Let $K_{i+1}=C_{3.1}^{i}\left(K_{1}\right), i=1,2,3$. Also suppose that $d_{\gamma(0)}(\alpha(0), \beta(0))=M$.

Lemma 5.12. Ladders of type 1: For $K_{1}, D, D_{1}$ as above and $M \geq D_{1}$, there exists $n_{1}=n_{1}\left(K_{1}, M\right)$ such that $\max \left\{d_{\gamma(-t)}(\alpha(-t), \beta(-t)), d_{\gamma(t)}(\alpha(t), \beta(t))\right\} \geq 8 M$ for all $t \geq n_{1}$.

Proof. Let $D_{2}=C_{5.9}\left(K_{1}, M\right)$ and let $C_{1}:=1+2.95 .10\left(M, D_{2}, K_{1}, \delta\right)$. If $d\left(\alpha\left(C_{1}\right), \beta\left(C_{1}\right)\right) \geq$ $D_{2}$ then for all $t \geq 0$ the length of the horizontal geodesic joining $\alpha\left(C_{1}+t\right)$ to $\beta\left(C_{1}+t\right)$ is greater than or equal to $A_{1} \cdot b_{1}^{t}$ for some $A_{1}=A_{5.9}\left(K_{1}, M\right), b_{1}=$ $45.9\left(K_{1}, M\right)$. Choose $t_{1}>0$ such that for all $t \geq t_{1}, A_{1} \cdot b_{1}^{t_{1}} \geq 8 M$.

Else, suppose $d\left(\alpha\left(C_{1}\right), \beta\left(C_{1}\right)\right)<D_{2}$. In this case, by Lemma 5.10 $d\left(\alpha\left(\frac{C_{1}-1}{2}\right), \beta\left(\frac{C_{1}-1}{2}\right)\right) \leq$ $D$. By the choice of the constants $D, D_{2}$ we can again apply Lemma [5.9] so that for all $t \geq 0$ the length of a horizontal geodesic $Y \cap F_{\gamma(-t)}$ is greater than or equal to $A_{2} \cdot b_{2}^{t}$, where the constants $A_{2}, b_{2}$ depend on $K_{1}$ and $D$. Choose $t_{2}>0$ such that for all $t \geq t_{2}, A_{2} \cdot b_{2}^{t} \geq 8 M$. Now let $n_{1}=\max \left\{C_{1}+t_{1}, t_{2}\right\}$. Thus we have $\max \left\{d_{\gamma(-t)}(\alpha(-t), \beta(-t)), d_{\gamma(t)}(\alpha(t), \beta(t))\right\} \geq 8 M$ for all $t \geq n_{1}=n_{1}\left(M, K_{1}\right)$.

Lemma 5.13. Ladders of type 2: Suppose $l>0$ and that for any $s \in[0, M-1]$ there is a $K_{2}$ - qi lift $\alpha_{1}$ of $\gamma$ in $Y$ through $\eta(s)$ such that $d\left(\alpha(t), \alpha_{1}(t)\right) \leq l$ for some $t \in[-L, L]$. There are $n_{2}=n_{2}\left(K_{1}, l\right)$ and $D_{4}=D_{4}\left(K_{1}, l\right)$ such that for all $t \geq n_{2}$ we have

$$
\max \left\{d_{\gamma(t)}(\alpha(t), \beta(t)), d_{\gamma(-t)}(\alpha(-t), \beta(-t))\right\} \geq 8 M \text { if } M \geq D_{4}+1 .
$$

Proof. Let $C_{3}=C_{5.9}\left(K_{3}, l\right), A_{3}=A \overline{5.9}\left(K_{3}, l\right), b_{3}=4 \overline{5.9}\left(K_{3}, l\right)$. Let $m_{0}:=$ $\min \left\{m \in \mathbb{N}: A_{3} \cdot b_{3}^{m} \geq D \overline{3.12}\left(g\left(2 K_{3}\right)\right)\right\}$, where $g$ refers to the function defined 
in Lemma 1.13. It follows easily from the bounded flaring condition that there is a constant $D_{4}^{\prime}$ such that the following is true:

Suppose we have two $K_{3}$-qi lifts $\alpha^{\prime}, \alpha^{\prime \prime}:[-L, L] \rightarrow X$ of the geodesic $\gamma$ : $[-L, L] \rightarrow B$ with $d_{\gamma(0)}\left(\alpha^{\prime}(0), \alpha^{\prime \prime}(0)\right) \geq D_{4}^{\prime}$ then $d\left(\alpha^{\prime}(t), \alpha^{\prime \prime}(t)\right) \geq D \overline{3.12}\left(g\left(2 K_{3}\right)\right)$ for all $t \in\left[0, m_{0}\right]$.

Let $D_{4}=\max \left\{D_{4}^{\prime}, C_{3}\right\}$ and let $M-1=N . D_{4}+r$ where $N \in \mathbb{N}$ and $0 \leq r<D_{4}$. Now construct a $K_{2}$-qi section $\beta_{1}$ in the ladder $Y$ such that $d_{\gamma(0)}\left(\beta(0), \beta_{1}(0)\right)=r+1$ and $d\left(\alpha\left(t_{0}\right), \beta_{1}\left(t_{0}\right)\right) \leq l$ for some $t_{0} \in[-L, L]$. Without loss of generality we assume that $t_{0} \in[-L, 0]$. We now use Lemma 5.10 (2) to break the subladder of $Y$, formed by $\alpha$ and $\beta_{1}$, by $K_{3}$-qi lifts $\alpha_{0}=\alpha, \alpha_{1}, \cdots, \alpha_{N}=\beta_{1}$ of $\gamma$ such that $d_{\gamma(0)}\left(\alpha_{i}(0), \alpha_{i+1}(0)\right)=D_{4}$. We have $d\left(\alpha_{i}\left(t_{0}\right), \alpha_{i+1}\left(t_{0}\right)\right) \leq l$. Thus by the choice of the constant $D_{4}, d_{\gamma(t)}\left(\alpha_{i}(t), \alpha_{i+1}(t)\right) \geq \max \left\{D \overline{3.12}\left(g\left(2 K_{3}\right)\right), A_{3} . b_{3}^{t}\right\}$ for all $t \geq 0$. Also (as in Step 2 of the proof of Proposition [3.14) $\cup\left[\alpha_{i}(t), \alpha_{i+1}(t)\right]$ is a partition of the horizontal geodesic segment $\left[\alpha(t), \beta_{1}(t)\right] \subset Y \cap F_{\gamma(t)}$, for all $t \in\left[0, m_{0}\right]$. Therefore, we can choose $n_{2}=n_{2}\left(K_{1}, l\right)$ such that for all $t \geq n_{2}$,

$$
\max \left\{d_{\gamma(t)}(\alpha(t), \beta(t)), d_{\gamma(-t)}(\alpha(-t), \beta(-t))\right\} \geq 8 M \text { if } M-1 \geq D_{4}=D_{4}\left(K_{1}, l\right)
$$

Flaring of general ladders: In the general case, first of all, we break the ladder $Y$ into subladders of special types as described above (see figure below where horizontal and vertical directions have been interchanged for aesthetic reasons).

Let us assume that $Y$ is bounded by $K-$ qi lifts $\alpha, \beta$ of a geodesic $\gamma:[-L, L] \rightarrow$ $X$. Let $\eta:[0, M] \rightarrow F_{\gamma(0)}$ be the geodesic $Y \cap F_{\gamma(0)}$. Let $K_{i}=C_{B 3.1}^{i}(K)$, and $l=$ $D \overline{3.12}\left(g\left(2 . K_{2}\right)\right)$. Let $M_{K}:=\max \left\{D_{1}\left(K_{1}\right), D_{4}\left(K_{1}, l\right)\right\}$, and $n_{K}:=\max \left\{n_{1}\left(K_{1}, M_{K}\right), n_{2}\left(K_{1}, l\right)\right\}$ where the functions $D_{1}, D_{4}, n_{1}, n_{2}$ are as in the proof of the flaring for the special ladders.

Claim: If $M \geq M_{K}$ then we have

$$
\max \left\{d_{\gamma\left(-n_{K}\right)}\left(\alpha\left(-n_{K}\right), \beta\left(-n_{K}\right)\right), d_{\gamma\left(n_{K}\right)}\left(\alpha\left(n_{K}\right), \beta\left(n_{K}\right)\right)\right\} \geq 2 . d_{\gamma(0)}(\alpha(0), \beta(0)) .
$$

To show this we inductively construct $K_{1}$-qi sections $\alpha_{0}=\alpha, \alpha_{1}, \cdots, \alpha_{i}=\beta$ in $Y$ to decompose it into subladders of the two types we mentioned above. This is done as in Step 1 of the proof of Proposition 3.14. Nevertheless we include a sketch of the argument for completeness.

Since $M \geq M_{K}$, therefore by Lemma 5.11 (2), we can construct a $K_{1}$-qi section $\alpha_{1}$ through $\eta\left(M_{K}\right)$. Now, suppose $\alpha_{1}, \cdots, \alpha_{j}$ has been constructed through the points $\eta\left(s_{1}\right), \cdots, \eta\left(s_{j}\right)$ respectively. If $d_{\gamma(0)}\left(\alpha_{j}(0), \beta(0)\right) \leq M_{K}$ define $\alpha_{j+1}=\beta$. Otherwise, if there is a $K_{1}$-qi section through $\eta\left(M_{K}+s_{j}\right)$ in the ladder formed by $\alpha_{j}$ and $\beta$, define it to be $\alpha_{j+1}$. If neither happens then consider the following set:

$\mathcal{T}_{j}=\left\{t \geq s_{j}+M_{K}: \exists\right.$ a $K_{1}$-qi section through $\eta(t)$ entering the ladder formed by $\alpha_{j}$ and $\left.\alpha\right\}$

Let $t_{j}=\sup \mathcal{T}_{j}$ be the supremum of this set. Define $\alpha_{j+1}$ to be a $K_{1}$-qi section through $s_{j+1}:=t_{j}+1$, in the ladder formed by $\alpha_{j}$ and $\beta$ that does not enter the ladder formed by $\alpha_{j}$ and $\alpha$. 


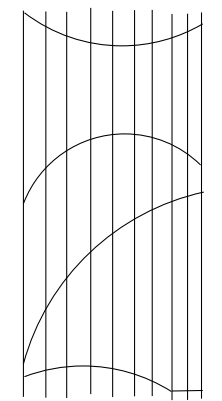

Flaring subladders

For each $j, \alpha_{j}$ and $\alpha_{j+1}$ form a special ladder (except possibly for the last one) and hence it must flare. Thus $\eta$ can be expressed as the disjoint union of subsegments that flare to the left and the union of the subsegments that flare to the right respectively. The total length of one of these types must be at least one-fourth of the length of $\eta$. The claim follows.

The first statement of Proposition 5.8 follows immediately. The last statement follows from Example 1.8 and the first part of this Proposition.

5.4. An Example. Let (Teich $\left.(S), d_{T}\right)$ be the Teichmuller space of a closed surface $S$ equipped with the Teichmuller metric $d_{T}$. Teichmuller space can also be equipped with an electric metric $d_{e}$ by electrocuting the thin parts (see Far98 for details on electric geometry and the introduction to this paper for a quick summary and relevant notation). Note (as per work of Masur-Minsky MM99, see also Mj09) that $\left(\right.$ Teich $\left.(S), d_{e}\right)$ is quasi-isometric to the curve complex $C C(S)$. Let $E:\left(T e i c h(S), d_{T}\right) \rightarrow\left(\right.$ Teich $\left.(S), d_{e}\right)$ be the identity map from the Teichmuller space of $S$ equipped with the Teichmuller metric $d_{T}$ to the Teichmuller space of $S$ equipped with the electric metric $d_{e}$.

We shall need the following Theorem due to Hamenstadt Ham10] which used an idea of Mosher Mos03] in its proof.

Theorem 5.14. Hamenstadt [Ham10]: For every $L>1$ there exists $D>0$ such that the following holds.

Let $f: \mathbb{R} \rightarrow\left(\right.$ Teich $\left.(S), d_{T}\right)$ be a Teichmuller L-quasigeodesic such that $E \circ f$ : $\mathbb{R} \rightarrow\left(\right.$ Teich $\left.(S), d_{E}\right)$ is also an L-quasigeodesic. Then for all $a, b \in \mathbb{R}$ there is a Teichmuller geodesic $\eta_{a b}$ joining $f(a), f(b) \in$ Teich $(S)$ such that the Hausdorff distance $d_{H}\left(f([a, b]), \eta_{a b}\right) \leq D$.

We are now in a position to prove a rather general combination proposition for metric bundles over quasiconvex subsets of $C C(S)$. For $j: K \rightarrow\left(\operatorname{Teich}(S), d_{T}\right)$ a map, let $U(S, K)$ denote the pullback (under $j$ ) of the universal curve over Teich $(S)$ equipped with the natural path metric. Also, the universal cover of the universal curve over Teich $(S)$ is a hyperbolic plane bundle over Teich $(S)$. Let $\widetilde{U(S, K)}$ denote the pullback to $K$ of this hyperbolic plane bundle.

Proposition 5.15. Let $\left(K, d_{K}\right)$ be a hyperbolic metric space satisfying the following:

There exists $C>0$ such that for any two points $u, v \in K$, there exists a bi-infinite $C$-quasigeodesic $\gamma \subset K$ with $d_{K}(u, \gamma) \leq C$ and $d_{K}(v, \gamma) \leq C$.

Let $j: K \rightarrow\left(\right.$ Teich $\left.(S), d_{T}\right)$ be a quasi-isometric embedding such that $E \circ j: K \rightarrow$ 
$\left(\right.$ Teich $\left.(S), d_{e}\right)$ is also a quasi-isometric embedding. Then $\widetilde{U(S, K)}$ is a hyperbolic metric space.

Proof. Clearly, $\widetilde{U(S, K)}$ is a metric bundle over $K$ (since the universal curve over Teich $(S)$ is topologically a product $S \times \operatorname{Teich}(S)$ and the latter is equipped with a foliation by totally geodesic copies of Teich $(S))$. Hence, by Theorem 4.3 it suffices to prove flaring. Let $S_{x}$ denote the fiber of $U(S, K)$ over $x \in i(K)$.

Let $[a, b]$ be a geodesic segment of sufficiently large length in $K$. By the hypothesis on $K$, there exists a bi-infinite geodesic passing within a bounded neighborhood of $[a, b]$. Hence without loss of generality, we may assume that $a, b$ lie on a bi-infinite geodesic in $K$.

Since $j$ and $E \circ j$ are both quasi-isometric embeddings, it follows that there exists $\epsilon>0$ such that for all $x \in K$, the injectivity radius of $j(x) \in \operatorname{Teich}(S)$ is greater than $\epsilon$. We shall refer to geodesics lying in the $\epsilon-$ thick part of Teich $(S)$ as fat Teichmuller geodesics. By Theorem 5.14 we may assume that $j(a), j(b)$ lie in a uniformly bounded neighborhood of a fat Teichmuller geodesic $\eta_{a b}$ whose endpoints in the Thurston boundary $\partial \operatorname{Teich}(S)$ are two singular foliations $\mathcal{F}_{+}, \mathcal{F}_{-}$. Let $d_{s}$ be the singular Euclidean metric on $S$ induced by the pair of singular foliations $\mathcal{F}_{+}, \mathcal{F}_{-}$.

The rest of the argument follows an argument of Mosher Mos97. Let $x$ be some point on the fat Teichmuller geodesic $\eta_{a b}$ obtained in the previous paragraph. Given any geodesic segment $\lambda \subset \widetilde{S_{x}}$ of length $l(\lambda)$, there are two projections $\lambda_{+}$and $\lambda_{-}$ onto (the universal covers of ) $\mathcal{F}_{+}, \mathcal{F}_{-}$in $\widetilde{S_{x}}$. At least one of these projections is of length at least $\frac{l(\lambda)}{2}$. If $u, v$ are two points on either side of $x$ such that $d_{T}(u, x) \geq m$ and $d_{T}(v, x) \geq m$, then the length of $\lambda$ in at least one of $\widetilde{S_{u}}$ and $\widetilde{S_{v}}$ is greater than $\frac{l(\lambda)}{2}\left(e^{m}\right)$.

Since all the surfaces with piecewise Euclidean metric involved in the above argument can be chosen to have uniformly bounded diameter, their universal covers are all uniformly quasi-isometric to a fixed Cayley graph of $\pi_{1}(S)$. Flaring follows.

The same proof goes through for $S^{h}-$ a finite area surface with cusps, provided we equip the cusps of $S^{h}$ with a zero metric. (This is the electric metric on cusps in the terminology of Far98.) Flaring in this situation is proved in Section 4.4 of MR08. The next proposition states this explicitly assuming that $S^{h}$ (resp. the universal cover $\widetilde{S^{h}}$ ) comes equipped with the zero metric on cusps (resp. lifts to $\left.\widetilde{S^{h}}\right)$.

Proposition 5.16. Let $\left(K, d_{K}\right)$ be a hyperbolic metric space satisfying the following:

There exists $C>0$ such that for any two points $u, v \in K$, there exists a bi-infinite $C$-quasigeodesic $\gamma \subset K$ with $d_{K}(u, \gamma) \leq C$ and $d_{K}(v, \gamma) \leq C$.

Let $j: K \rightarrow\left(\right.$ Teich $\left.\left(S^{h}\right), d_{T}\right)$ be a quasi-isometric embedding such that $E \circ j: K \rightarrow$ $\left(\right.$ Teich $\left.\left(S^{h}\right), d_{e}\right)$ is also a quasi-isometric embedding. Then $\widetilde{U\left(S^{h}, K\right)}$ is a hyperbolic metric space.

From Proposition 5.16 we obtain directly the following consequence (see [FM02 for definitions). 
Consider a surface $S^{h}$ with finitely many punctures. Let $K=\pi_{1}\left(S^{h}\right)$ and let $\mathcal{K}=\left\{K_{1}, \cdots, K_{n}\right\}$ be the collection of peripheral subgroups. The pure mapping class group is the subgroup of the mapping class group that preserves individual punctures.

Proposition 5.17. Let $K=\pi_{1}\left(S^{h}\right)$ be the fundamental group of a surface with finitely many punctures and let $K_{1}, \cdots, K_{n}$ be its peripheral subgroups. Let $Q$ be a convex cocompact subgroup of the pure mapping class group of $S^{h}$. Let

$$
1 \rightarrow K \rightarrow G \stackrel{p}{\rightarrow} Q \rightarrow 1
$$

and

$$
1 \rightarrow K_{i} \rightarrow N_{G}\left(K_{i}\right) \stackrel{p}{\rightarrow} Q_{i} \rightarrow 1
$$

be the induced short exact sequences of groups. Then $G$ is strongly hyperbolic relative to the collection $\left\{N_{G}\left(K_{i}\right)\right\}, i=1, \cdots, n$.

Conversely, if $G$ is (strongly) hyperbolic relative to the collection $\left\{N_{G}\left(K_{i}\right)\right\}, i=$ $1, \cdots, n$, then $Q$ is convex-cocompact.

Proof. Suppose that $Q$ is convex cocompact. Then $Q$ is hyperbolic by FM02, KL08. Also, by Theorem 5.2, $Q=Q_{i}$ for all $i$. This is because $K$ is strongly hyperbolic relative to $K_{i}$ for each $i=1, \cdots, n$. Let $\mathcal{E}\left(G, K_{1}, \cdots, K_{n}\right)$ denote the electric space obtained from (the Cayley graph of) $G$ after coning off translates of (the Cayley graphs of) $K_{i}$ for all $i=1, \cdots, n$. Then $\mathcal{E}\left(G, K_{1}, \cdots, K_{n}\right)$ is a metric graph bundle quasi-isometric to a $\widehat{K}\left(=\mathcal{E}\left(K, K_{1}, \cdots, K_{n}\right)\right)$-bundle over $Q$ where $\widehat{K}$ denotes $K$ with copies of $K_{i}$ coned off for all $i=1, \cdots, n$. . The flaring condition for this bundle and hence weak relative hyperbolicity of the pair $\left(G,\left\{K_{1}, \cdots, K_{n}\right\}\right)$ follow from Proposition 5.16 .

Let $\mathcal{Q}_{i}$ denote the collection of translates of (Cayley graphs of) $Q\left(=N_{G}\left(K_{i}\right) / K_{i}\right)$ in $\mathcal{E}\left(G, K_{1}, \cdots, K_{n}\right)$, where each copy of $Q$ in $\mathcal{E}\left(G, K_{1}, \cdots, K_{n}\right)$ is a copy of the electric space $\mathcal{E}\left(N_{G}\left(K_{i}\right), K_{i}\right)$ obtained by coning off $K_{i}$ in translates of (Cayley graphs of) $N_{G}\left(K_{i}\right)$. Let $\mathcal{Q}=\cup_{i} \mathcal{Q}_{i}$.

To prove that $G$ is strongly hyperbolic relative to the $N_{G}\left(K_{i}\right)$ 's, it suffices to prove that $\mathcal{E}\left(G, K_{1}, \cdots, K_{n}\right)$ is strongly hyperbolic relative to $\mathcal{Q}$, as $K$ is already strongly hyperbolic relative to $K_{1}, \cdots, K_{n}$ by Far98. That $\mathcal{E}\left(G, K_{1}, \cdots, K_{n}\right)$ is strongly hyperbolic relative to $\mathcal{Q}$ would in turn follow Mj11 from (uniform) mutual coboundedness of pairs of elements in $\mathcal{Q}$. Note also that each $Q_{j} \in \mathcal{Q}$ is quasiisometrically embedded and hence a quasiconvex subset of $\mathcal{E}\left(G, K_{1}, \cdots, K_{n}\right)$. Any two such $Q_{1}, Q_{2}$ 's define a ladder $C\left(Q_{1}, Q_{2}\right)$ by regarding $Q_{1}, Q_{2}$ as qi sections of the metric graph bundle $\mathcal{E}\left(G, K_{1}, \cdots, K_{n}\right)$ over $Q$. Each $C\left(Q_{1}, Q_{2}\right)$ is hyperbolic by Proposition 3.14. Hence, the ladder $C\left(Q_{1}, Q_{2}\right)$ also satisfies flaring by Proposition 5.8 .

To establish mutual coboundedness, we argue by contradiction. Let $d_{h}$ denote the horizontal distance in $\mathcal{E}\left(G, K_{1}, \cdots, K_{n}\right)$. Suppose that elements of the collection $\mathcal{Q}$ do not satisfy (uniform) mutual coboundedness. Then there exists $D_{0}>0$ such that for any $l>0$, there exists a pair $Q_{1}, Q_{2} \in \mathcal{Q}$ and a geodesic segment $r:[0, l] \rightarrow Q$ such that $d_{h}\left(s_{1} \circ r(t), s_{2} \circ r(t)\right) \leq D_{0}$ for all $t \in[0, l]$, where $s_{i}: Q \rightarrow \mathcal{E}\left(G, K_{1}, \cdots, K_{n}\right)$ are quasi-isometric embeddings defining the sections $Q_{1}, Q_{2}$. Since the number of elements in $K$ of length at most $D_{0}$ is bounded it follows that for sufficiently large $l$, there exists $q \in Q, q \neq 1$ and a non-peripheral element $h \in K$ such that $s(q), h$ commute. This is impossible for $Q$ convex cocompact, proving the forward direction of the Proposition. 
We now prove the converse direction. Hyperbolicity of $Q$ follows from Proposition 5.7 To prove convex cocompactness, it is enough to show by FM02 that some orbit of the action of $Q$ on $\left(\operatorname{Teich}(S), d_{T}\right)$ is quasiconvex.

Since $G$ is strongly hyperbolic relative to the collection $\left\{N_{G}\left(K_{i}\right)\right\}$, it follows from Lemma 1.50 that $\mathcal{E}\left(G, K_{1}, \cdots, K_{n}\right)$ is strongly hyperbolic relative to the collection $\mathcal{Q}$ of translates of (Cayley graphs of) $Q\left(=N_{G}\left(K_{i}\right) / K_{i}\right)$ in $\mathcal{E}\left(G, K_{1}, \cdots, K_{n}\right)$ as defined above. Since $Q$ is hyperbolic, it follows (cf. Bow97 Section 7) that $\mathcal{E}\left(G, K_{1}, \cdots, K_{n}\right)$ is hyperbolic. Thus, $\mathcal{E}\left(G, K_{1}, \cdots, K_{n}\right)$ is a hyperbolic metric graph bundle over $Q$. Hence, from Proposition [5.8 the bundle $\mathcal{E}\left(G, K_{1}, \cdots, K_{n}\right)$ over $Q$ satisfies flaring. The logarithm of the stretch factor guaranteed by flaring gives a lower bound on the Teichmuller distance.

The remainder of the argument is an exact replica of the proof of Theorem 1.2 of FM02 (Section 5.2 of FM02 in particular), which proves the analogous statement for surfaces without punctures. We do not reproduce the argument here but point out that the only place in the proof where explicit use is made of closedness of $S$ is Theorem 5.5 of [FM02, which, in turn is taken from [Mos03]. A straightforward generalization of this fact to the punctured surface case is given in [Pal11].

Remark 5.18. It is worth noting that a group $G$ as in Proposition 5.17 cannot act freely, properly discontinuously by isometries on a Hadamard manifold of pinched negative curvature unless $Q$ is virtually cyclic, as the normalizer $N_{G}\left(K_{i}\right)$ is not nilpotent.

As an application of Proposition 5.15 we give the first examples of surface bundles over hyperbolic disks, with Gromov-hyperbolic universal cover. It has been an open question (cf. KL08, FM02]) to find purely pseudo Anosov surface groups in $M C G(S)$. The example below is a step towards this.

In [LS11 Leininger and Schleimer construct examples of disks $\left(Q, d_{Q}\right)$ quasiisometric to $\mathbb{H}^{2}$ and quasi-isometric embeddings $j: Q \rightarrow\left(\right.$ Teich $\left.(S), d_{T}\right)$ such that $E \circ j: Q \rightarrow\left(\operatorname{Teich}(S), d_{e}\right)$ is also a quasi-isometric embedding. By Proposition 5.15, the hyperbolic plane bundle $\widetilde{U(S, Q)}$ is a hyperbolic metric space.

A brief sketch of Leininger-Schleimer's construction [LS11 follows:

The curve complex $C C(S, x)$ of a surface with one puncture (or equivalently, a marked point $x)$ admits a surjective map to $C C(S)$ such that the fiber over $\eta \in$ $C C(S)$ is the Bass-Serre tree of the splitting of $\pi_{1}(S)$ over the cyclic groups represented by the simple closed curves in $\eta$. Suppose $\gamma$ is a bi-infinite geodesic in $C C(S)$ coming from a geodesic in Teich $(S)$ lying in the thick part. Inside $C C(S, x)$ one has the space of trees over $\gamma$, and the authors of [LS11] construct lines in each tree over $\gamma$ whose union $Q_{1}$ is quasi-isometric to the hyperbolic plane. Using a branched cover-trick, they construct from $Q_{1}$ a new disk $Q \subset C C\left(S^{\prime}\right)$ (for a closed surface $S^{\prime}$, which is a branched cover of $S$ branched over the marked point of $S$ ) such that $Q$ satisfies the hypotheses of Proposition 5.15 .

\section{REFERENCES}

$\left[\mathrm{ABC}^{+} 91\right]$ J. Alonso, T. Brady, D. Cooper, V. Ferlini, M. Lustig, M. Mihalik, M. Shapiro, and H. Short, Notes on word hyperbolic groups, Group Theory from a Geometrical Viewpoint (E. Ghys, A. Haefliger, A. Verjovsky eds.) (1991), 3-63.

[BF92] M. Bestvina and M. Feighn, A Combination theorem for Negatively Curved Groups, J. Differential Geom., vol 35 (1992), 85-101. 
[BH99] M. Bridson and A Haefliger, Metric spaces of nonpositive curvature, Grundlehren der mathematischen Wissenchaften, Vol 319, Springer-Verlag (1999).

[Bow97] B. H. Bowditch, Relatively hyperbolic groups, preprint, Southampton (1997).

[Bow07] , The Cannon-Thurston map for punctured surface groups, Math. Z. 255 $((2007)), 35-76$.

[CDP90] M. Coornaert, T. Delzant, and A. Papadopoulos, Geometrie et theorie des groupes, Lecture Notes in Math., vol.1441, Springer Verlag (1990).

[CT85] J. Cannon and W. P. Thurston, Group Invariant Peano Curves, preprint, Princeton (1985).

[CT07] Group Invariant Peano Curves, Geom. Topol. 11 (2007), 1315-1355.

[Far98] B. Farb, Relatively hyperbolic groups, Geom. Funct. Anal. 8 (1998), 810-840.

[FM02] B. Farb and L. Mosher, Convex cocompact subgroups of mapping class groups, Geom. Topol. 6 (2002), 91-152.

[Gd90] E. Ghys and P. de la Harpe(eds.), Sur les groupes hyperboliques d'apres Mikhael Gromov, Progress in Math. vol 83, Birkhauser, Boston Ma. (1990).

[Gro85] M. Gromov, Hyperbolic Groups, in Essays in Group Theory, ed. Gersten, MSRI Publ.,vol.8, Springer Verlag (1985), 75-263.

[Gro93] _ Asymptotic Invariants of Infinite Groups, in Geometric Group Theory,vol.2; Lond. Math. Soc. Lecture Notes 182, Cambridge University Press (1993).

[Ham05] U. Hamenstadt, Word hyperbolic extensions of surface groups, preprint, arXiv:math/0505244 (2005).

[Ham07] _ Geometry of complex of curves and teichmuller spaces, in Handbook of Teichmuller Theory Vol. 1, EMS (2007), 447-467.

[Ham10] , Stability of Quasigeodesics in Teichmuller Space, Geom. Dedicata 146 (2010), 101-116.

[Kap08] M. Kapovich, Problems on Boundaries of groups and Kleinian Groups, http://www.aimath.org/pggt/Boundaries boundaries-version4.pdf (2008).

[KL08] R. P. KentIV and C. Leininger, Shadows of mapping class groups: capturing convex cocompactness, Geom. Funct. Anal. 18 (2008), 1270-1325.

[LMS11] C. Leininger, M. Mj, and S. Schleimer, The universal Cannon-Thurston maps and the boundary of the curve complex, Comment. Math. Helv. 86(4), arXiv:0808.3521 (2011), 769-816.

[LS11] C. Leininger and S. Schleimer, Hyperbolic spaces in Teichmuller spaces, arXiv:1110.6526, preprint (2011).

[Mit97] M. Mitra, Ending Laminations for Hyperbolic Group Extensions, Geom. Funct. Anal. 7 (1997), 379-402.

[Mit98a] Cannon-Thurston Maps for Hyperbolic Group Extensions, Topology 37 (1998), 527-538.

[Mit98b] C Cannon-Thurston Maps for Trees of Hyperbolic Metric Spaces, J. Differential Geom. 48 (1998), 135-164.

[Mj06] M. Mj, Cannon-Thurston Maps for Surface Groups, preprint, arXiv:math.GT/0607509 (2006).

[Mj09] - Mapping class groups and interpolating complexes: Rank, J. Ramanujan Math. Soc. 24, no. 4 (2009), 341-357.

[Mj11] Cannon-Thurston Maps, i-bounded Geometry and a Theorem of McMullen, Actes du séminaire Théorie spectrale et géométrie 28, Année 2009-10, arXiv:math.GT/0511104 (2011), 63-108.

[MM99] H. A. Masur and Y. N. Minsky, Geometry of the complex of curves I: Hyperbolicity, Invent. Math.138 (1999), 103-139.

[Mos96] L. Mosher, Hyperbolic Extensions of Groups, J. Pure Appl. Algebra 110(3) (1996), 305-314.

[Mos97] _ A hyperbolic-by-hyperbolic hyperbolic group, Proc. Amer. Math. Soc. 125 (1997), 3447-3455.

[Mos03] _ Stable Teichmuller quasigeodesics and ending laminations, Geom. Topol. 7 (2003), 33-90.

[MP11] M. Mj and A. Pal, Relative Hyperbolicity, Trees of Spaces and Cannon-Thurston Maps, arXiv:0708.3578, Geom. Dedicata 151(1) (2011), 59-78. 
[MR08] M. Mj and L. Reeves, A Combination Theorem for Strong Relative Hyperbolicity, Geom. Topol. 12 (2008), 1777- 1798.

[Pal10] A. Pal, Relatively Hyperbolic Extensions of Groups and Cannon-Thurston Maps, Proc. Indian Acad. Sci. Math. Sci. 120 (1), arXiv:0801.0933 (2010), 57-68.

[Pal11] - Notes on Stable Teichmuller quasigeodesics, preprint, arXiv:1109.3794 (2011).

[Pap95] P. Papasoglu, Strongly geodesically automatic groups are hyperbolic, Invent. math. 121, (1995), 323- 334.

[Pap05] Luasi-isometry invariance of group splittings, Ann. Math., vol. 161, no.2 (2005), 759-830.

RKM Vivekananda University, Belur Math, WB-711 202, India 